\title{
WestVirginiaUniversity
}

THE RESEARCH REPOSITORY @ WVU

Graduate Theses, Dissertations, and Problem Reports

2014

\section{Neutral Hydrogen in the Local Group and around the Milky Way}

Spencer A. Wolfe

Follow this and additional works at: https://researchrepository.wvu.edu/etd

\section{Recommended Citation}

Wolfe, Spencer A., "Neutral Hydrogen in the Local Group and around the Milky Way" (2014). Graduate Theses, Dissertations, and Problem Reports. 6969.

https://researchrepository.wvu.edu/etd/6969

This Dissertation is protected by copyright and/or related rights. It has been brought to you by the The Research Repository @ WVU with permission from the rights-holder(s). You are free to use this Dissertation in any way that is permitted by the copyright and related rights legislation that applies to your use. For other uses you must obtain permission from the rights-holder(s) directly, unless additional rights are indicated by a Creative Commons license in the record and/ or on the work itself. This Dissertation has been accepted for inclusion in WVU Graduate Theses, Dissertations, and Problem Reports collection by an authorized administrator of The Research Repository @ WVU.

For more information, please contact researchrepository@mail.wvu.edu. 
Neutral Hydrogen in the Local Group and around the Milky Way

\author{
Spencer A. Wolfe
}

Dissertation submitted to the Eberly College of Arts and Sciences at West Virginia University in partial fulfillment of the requirements for the degree of

Doctor of Philosophy
in
Physics

Dr. D.J. Pisano, Ph.D., Chair

Dr. Paul Cassak, Ph.D.

Dr. Justin Legleiter, Ph.D.

Dr. Felix J. Lockman, Ph.D.

Dr. Duncan Lorimer, Ph.D.

Dr. Maura McLaughlin, Ph.D.

Department of Physics and Astronomy

Morgantown, West Virginia 2014

Keywords: High Velocity Clouds; Intergalactic Medium; Intermediate Velocity Clouds; Local Group; M31; M33; Milky Way

Copyright 2014 Spencer A. Wolfe 


\title{
ABSTRACT \\ Neutral Hydrogen in the Local Group and around the Milky Way
}

\author{
Spencer A. Wolfe
}

Galaxies in our universe must acquire fresh gas to continue forming new stars. A likely source of this material may be the gas that resides between galaxies. We do not, however, have a clear understanding of the specifics, such as its distribution. The first claimed detection of this "cosmic web" of material directly in emission was published a decade ago using the Westerbork Synthesis Radio Telescope in the Netherlands while surveying neutral hydrogen in the Local Group of galaxies. Later evidence, in the form of stellar surveys and test particle simulations, showed that a tidal origin of the gas was another possibility.

More recent survey work of the Local Group, specifically between the galaxies M31 and M33, motivated us to map a section of the Westerbork emission using the Robert C . Byrd Green Bank Telescope (GBT). Our survey covers a 12 square degree area between M31 and M33, in which we reach $21 \mathrm{~cm}$ column density sensitivities of $\sim 10^{17.2} \mathrm{~cm}^{-2}$ after 400 hours of observations. These observations provide more than a factor of five better spatial resolution, and better than a factor of three in velocity resolution. Not only do we confirm the emission seen in the Westerbork data, we find that the hydrogen gas is composed of clouds a few kiloparsecs across, with properties suggesting they are a unique population to the Local Group. We conclude that the clouds are likely transient condensations from an intergalactic filament of gas, although a tidal feature cannot currently be ruled out. We also conducted GBT pointings to the northwest of M31 to search for the extended emission seen in the Westerbork data as well. What detections we find appear to be more related to the high velocity cloud population of M31. We are continuing to map other regions around M31 to search for more diffuse emission.

We also present southern sky maps of the high velocity and intermediate velocity clouds around our own Milky Way, using $21 \mathrm{~cm}$ survey data from the Parkes telescope in Australia. The existence of these objects have been known for over 50 years, yet there is no general consensus as to their origins. The maps we have produced are the most detailed to date, with high spatial and velocity resolution and good sensitivity. By using a model of Milky Way rotation, we more effectively filter out foreground emission from our own Galaxy to produce these maps. We also discuss the basic global properties of this gas and the features that are seen. Apart from the Magellanic system and Galactic warp, most of the emission is in the form of small clouds. Some of the emission seen in these data may be representative of the eventual fate of the M31-M33 clouds. Work with this survey is ongoing. 


\section{Acknowledgments}

As this endeavor draws to a close, I wish to acknowledge those who have helped me reach this point.

During my second semester at WVU as an undergraduate, I transferred from engineering to the physics department and I would like to thank Dr. Martin Ferer, who became my undergraduate advisor and an excellent teacher. It was he who convinced me to go to graduate school.

I would also like to thank Dr. John Littleton whose astrophysics course, which was the only one being offered at the time, sparked my interest in astronomy and astronomical phenomena. I also would like to thank Drs. Earl Scime and Nancy Giles, who allowed me to stay at WVU as a graduate student.

I next want to thank my advisor Dr. D.J. Pisano. I knew early on in graduate school that I wanted to be involved with astrophysical research. The areas of interest he brought when coming to the department I found more interesting than any other offered, and still do. I would like to thank him for his time, patience and understanding, especially when having a student who began with a limited background in astronomy.

I wish to thank Jay Lockman at the NRAO in Green Bank. Many of the ventures presented in this work were inspired by his previous work and through spirited discussions with him. I also wish to thank all the scientific and support staff in Green Bank. The GBT is a wonderful instrument, but it is only as good as those who run and maintain it. The staff's support has proven invaluable.

I wish to thank my office mates, Katie Rabidoux and Andrew Seymour who were always a welcome source of support and discussion.

I close by thanking my parents, Terry and Debra Wolfe for their constant support and understanding. They never questioned my academic choices or interests and always knew that I would one day find a path in which I would succeed. 


\section{Table of Contents}

List of Tables $\quad$ vi

List of Figures vii

1 Introduction 1

1.1 The Basics of H i Radio Astronomy . . . . . . . . . . . . . . . . . . . 1

1.2 Gas Accretion onto Galaxies . . . . . . . . . . . . . . . . . 3

1.3 Diffuse H i Discovered in the Local Group of Galaxies . . . . . . . . . 4

1.4 High Velocity and Intermediate Velocity Clouds . . . . . . . . . . . . 6

1.5 The Baryonic Tully-Fisher Relation . . . . . . . . . . . . . . . 7

1.6 Thesis Outline . . . . . . . . . . . . . . . . . 8

2 Mapping Diffuse Neutral Hydrogen with the GBT 10

2.1 Mapping $\mathrm{H}_{\mathrm{r}}$ in the Cosmic Web . . . . . . . . . . . . . . . . 10

2.1.1 Background . . . . . . . . . . . . . . . . 10

2.1.2 GBT Mapping Sensitivity . . . . . . . . . . . . . . 11

2.2 Observing Method . . . . . . . . . . . . . . . . . . 14

2.2.1 Concept .......................... 14

2.2.2 Front End and Back End Configuration . . . . . . . . . . . . 15

2.2.3 Observing the Calibrator 3C48 . . . . . . . . . . . . . 16

2.2.4 Mapping the $\mathrm{H}_{\mathrm{I}}$. . . . . . . . . . . . . . . . . . . 16

2.3 Data Reduction . . . . . . . . . . . . . . . . . . . . . 18

2.3.1 Concept . . . . . . . . . . . . . . . 18

2.3.2 $\mathrm{T}_{\mathrm{CAL}}$ from $3 \mathrm{C} 48$. . . . . . . . . . . . . . . . . . . 19

2.3.3 Hi Mapping Reduction . . . . . . . . . . . . . . . . . . . 20

2.4 Blanking Issues with the Spectrometer . . . . . . . . . . . . . . . . . 24

2.5 Gridding the Spectral Line Data . . . . . . . . . . . . . . . 26

3 Discrete Clouds of Neutral Gas between the Galaxies M31 and M33 29

3.1 Introduction . . . . . . . . . . . . . . . . . . . . . . . . . . . . . . . 29

3.2 Preliminary Results . . . . . . . . . . . . . . . . . . . . 30

3.3 Virial Mass and Survival Times . . . . . . . . . . . . . . . . 36

3.4 Cloud Origins . . . . . . . . . . . . . . . . . . . . . . . . . . . . . . . . . . . . . . . 38

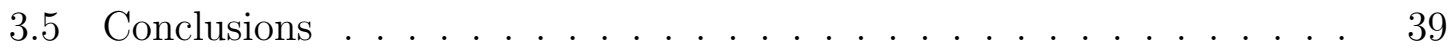

4 The Diffuse H i around M31 40

4.1 Observations. . . . . . . . . . . . . . . . . . . 40

4.2 Results . . . . . . . . . . . . . . . . . . . . 41

4.2.1 The 400 Hour Survey . . . . . . . . . . . . . . . . . . 41

4.2.2 Velocities and Linewidths . . . . . . . . . . . . . . . 43

4.2.3 The Pointings to the Northwest of M31 . . . . . . . . . . . 47

4.3 Discussion . . . . . . . . . . . . . . . . . 53

4.4 Conclusions . . . . . . . . . . . . . . . . . . . 58 
5 High and Intermediate Velocity H i in the Southern Sky 61

5.1 Background .......................... 61

5.2 The Galactic All-Sky Survey . . . . . . . . . . . . . . . . . . 64

5.3 Blanking Milky Way Emission . . . . . . . . . . . . . . 65

5.4 Creating Southern Sky Maps . . . . . . . . . . . . . . . . 68

5.4.1 Calculating the Column Density . . . . . . . . . . . . 68

5.4.2 Creating a Map of the Deviation Velocity . . . . . . . . . . . 70

5.5 Global Properties . . . . . . . . . . . . . . . . . 76

5.6 Individual Features . . . . . . . . . . . . . . . . . . . . . . . . . . . . . . . 79

5.6.1 High Velocity Emission . . . . . . . . . . . . . . . 79

5.6.2 Intermediate Velocity Emission . . . . . . . . . . . . . 81

5.7 Conclusions . . . . . . . . . . . . . . . . . 82

6 Conclusions and Future Work 84

6.1 The M31-M33 Hi clouds . . . . . . . . . . . . . . . . . . . . 84

6.2 HVCs and IVCs with GASS . . . . . . . . . . . . . . 86

A The Hi Hyperfine Transition 88

B Radiative Transfer and the H i Line 93

C Radio Telescope and Spectral Line Fundamentals $\quad 99$ 


\section{List of Tables}

3.1 Local Group H i Cloud Properties: 250 hours . . . . . . . . . . . . . . 34

4.1 Local Group H i Cloud Properties: 400 hours . . . . . . . . . . . . . . 44

4.2 Deep Pointings to the Northwest of M31 . . . . . . . . . . . . . 51 


\section{List of Figures}

2.1 GBT Local Group Mapping Areas . . . . . . . . . . . . . . . . . . 12

2.2 The Robert C. Byrd Green Bank Telescope . . . . . . . . . . . . . . 14

2.3 An Illustration of the GBT Observing Method . . . . . . . . . . . 15

2.4 GBT Pointings of $3 \mathrm{C} 48 \ldots \ldots \ldots \ldots \ldots \ldots \ldots$

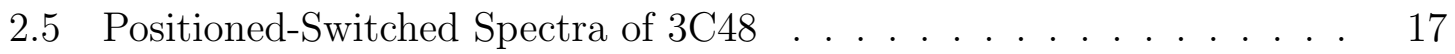

$2.6 \quad$ H I Mapping Spectra over One Integration $\ldots \ldots \ldots \ldots$

$2.7 \mathrm{~T}_{\mathrm{CAL}}$ values for the GBT Spanning 11 Months . . . . . . . . . 20

2.8 A Reduced H ı Mapping Spectrum . . . . . . . . . . . . . . . . 22

2.9 Histogram of Hi Spectral Noise over all Observing Sessions . . . . . . 23

2.10 Doppler Blanking Effect on GBT Exposure Times . . . . . . . . . 25

2.11 Doppler Blanking Effect as Seen in GBT10A-043 . . . . . . . . . 26

2.12 A Moment Zero Map of our Hi Survey Data . . . . . . . . . . 28

3.1 Channel Maps of the Local Group Clouds . . . . . . . . . . . . . . 32

3.2 Preliminary $21 \mathrm{~cm}$ Maps . . . . . . . . . . . . . . . . . . . 33

3.3 A Position-Velocity Plot of the Local Group Clouds . . . . . . . . . . 35

3.4 Baryonic Mass Versus Rotation Speed for the Local Group Clouds . . 37

4.1 GBT Pointing Locations . . . . . . . . . . . . . . . . . . 42

4.2 Final Map of the M31-M33 H I Clouds. . . . . . . . . . . . . 43

4.3 Velocity Field of the M31-M33 H I Clouds. . . . . . . . . . . . 44

4.4 Velocity Field of Cloud $1 \ldots \ldots \ldots \ldots$. . . . . . . . . . . . . . . . . .

4.5 Velocity Field of Clouds $2 \& 3 . \ldots \ldots \ldots \ldots$

4.6 Velocity Field of Cloud $4 . \ldots \ldots \ldots$

4.7 Velocity Field of Cloud $5 . \ldots \ldots \ldots$

4.8 PLCUB Plot for Cloud $1 \ldots \ldots \ldots \ldots$. . . . . . . . . . . . 47

4.9 PLCUB Plot for Clouds 2 and $3 \ldots \ldots \ldots \ldots \ldots$

4.10 PLCUB Plot for Cloud $4 \ldots \ldots \ldots \ldots$

4.11 PLCUB Plot for Cloud $5 \ldots \ldots \ldots \ldots$

4.12 PLCUB Plot for Cloud $6 \ldots \ldots \ldots \ldots$

4.13 PLCUB Plot for Cloud $7 \ldots \ldots \ldots \ldots \ldots$

4.14 GBT Pointing $\# 1 \ldots \ldots \ldots \ldots$

4.15 GBT Pointing \#2 $\ldots \ldots \ldots \ldots \ldots \ldots$

4.16 GBT Pointing \#4 . . . . . . . . . . . . . . . . 53

4.17 GBT Pointing $\# 7 \ldots \ldots \ldots \ldots \ldots \ldots$

4.18 GBT Pointing \#10 $\ldots \ldots \ldots \ldots \ldots \ldots \ldots$

4.19 GBT Pointing \#19 $\ldots \ldots \ldots \ldots \ldots$

4.20 Smith's Cloud . . . . . . . . . . . . . . . . . . . . 56

4.21 NAM modeling . . . . . . . . . . . . . . . . . . . . 57

4.22 Updated Tully-Fisher Plot . . . . . . . . . . . . . . . . . 57

4.23 LGSR Versus the Angular Distance from M31 . . . . . . . . . 59

5.1 Maximum and Minimum LSR Velocities due to Milky Way Rotation . 68 
5.2 An Example of Blanking Milky Way H I Emission . . . . . . . . . . . 69

5.3 Column Density Map of High Velocity Gas as Seen with GASS . . . . 71

5.4 Column Density Map of Intermediate Velocity Gas as Seen with GASS 72

5.5 Column Density Map of the High and Intermediate Velocity Gas as Seen with GASS . . . . . . . . . . . . . . . . . . . 73

5.6 Bicolor Map of the HVC and IVC gas. . . . . . . . . . . . . . . . . . 74

5.7 Deviation Velocity Map for Both the High Velocity and Intermediate Velocity Gas . . . . . . . . . . . . . . . . . . . 75

5.8 Column Density Distribution of the GASS Survey for the HVC Gas, IVC Gas and Both Populations Combined . . . . . . . . . . . . . . . 77

5.9 Deviation Velocity Distribution for Both the HVC and IVC gas. . . . 80

C.1 Raw Spectral Line Data . . . . . . . . . . . . . . . . . . . . . . 102

C.2 Spectral Line Difference . . . . . . . . . . . . . . . . . 103 


\section{Chapter 1}

\section{Introduction}

\subsection{The Basics of $\mathrm{H}_{\mathrm{I}}$ Radio Astronomy}

Radio astronomers can detect neutral hydrogen, or $\mathrm{HI}$, via the spin-flip transition. This was first predicted by van de Hulst in 1944 and was later confirmed by Ewen \& Purcell (1951). The proton and electron in the hydrogen atom are both spin $\frac{1}{2}$ particles. Thus the z-components of their spins can be either oriented parallel or antiparallel. The parallel state is at a slightly higher energy than the antiparallel. Thus, when the electron's spin flips from parallel to antiparallel, a photon of wavelength $21.1 \mathrm{~cm}$ and frequency of $1420.4 \mathrm{MHz}$ is emitted. See Appendix A for a detailed discussion. The amount of energy needed to excite the electron to the antiparallel state is quite small and the transition probability of the transition is also quite small, with a predicted decay time of $\mathrm{A}_{10}^{-1} \approx 10^{7} \mathrm{yr}$. Even so, the amount of H I present in the Galaxy and other sources is large enough that $21 \mathrm{~cm}$ emission can easily be seen by radio telescopes.

Because we know the transition frequency of the $21 \mathrm{~cm}$ line quite well, we can use the Doppler effect to easily determine a line-of-sight velocity to the $\mathrm{H}$ i emission. This allows us to determine the kinematics of hydrogen gas not only in our own Galaxy, but many external galaxies and extragalactic sources as well. Velocity gradients across a source, such as a cloud or galaxy, may be due to rotation or stretching of the gas. 
If the radio source is extended (larger than the telescope beam), then the brightness temperature $T_{B}$ is typically used as a measure of the intensity. The brightness temperature is the temperature the object would have if it were a blackbody producing the intensity of the radiation we observe in the Rayleigh-Jeans limit. Brightness temperature only represents the temperature $\mathrm{T}$ if the gas is optically thick. It is typical in $\mathrm{H}$ I astronomy that we assume the gas is instead optically thin, in which case $\mathrm{T}_{\mathrm{B}} \sim \tau_{\nu} \mathrm{T}$, where $\tau_{\nu}$ is the optical depth. See Appendix B for a more detailed discussion of brightness temperature and optical depth.

If one assumes that the $\mathrm{H}_{\mathrm{I}}$ is optically thin, then it can be shown (see Wilson et al., 2009, Appendix B) that the column density of the emission is directly proportional to the integrated brightness temperature.

$$
\mathrm{N}_{\mathrm{HI}}=1.822 \times 10^{18} \int_{\text {line }}\left(\frac{\mathrm{T}_{\mathrm{B}}(\mathrm{v})}{\mathrm{K}}\right)\left(\frac{\mathrm{dv}}{\mathrm{km} \mathrm{s}^{-1}}\right) \mathrm{cm}^{-2} .
$$

If one uses a telescope to map a given area, then the spectral line data can be gridded into a data cube, with the first two axes being spatial and third being either velocity or frequency. One can then make maps of the the total intensity (moment 0 ), velocity as a function of position (moment 1 or velocity field) and such. Intensity maps can help one search for extended structures and velocity fields can help one investigate the kinematics. 


\subsection{Gas Accretion onto Galaxies}

The current understanding of galaxy evolution is that galaxies assemble most of their mass, via dark matter, through a hierarchical process of mergers and accretion events (e.g Press \& Schechter, 1974) over cosmic times. These dark matter halos should then accrete gas from the surrounding intergalactic medium (IGM), which then cools and becomes stars (e.g. White \& Frenk, 1991), supplying fuel for star formation for a few billion years.

The star formation rate of the universe peaked at a redshift of about 1.5 and has decreased since (Madau et al., 1998). The cosmological redshift is the increase in wavelength of emission from distant astronomical objects due to the cosmological expansion of the universe and is related to how long ago the observed light was emitted from the source. A redshift of 1.5 corresponds to about 9 Gyr ago. The Hi content of galaxies, however, has remained constant for the past 11 billion years (Prochaska et al., 2005), even though the intensified amount of star formation in the past should have consumed most of their accumulated Hi. One explanation is that the galaxies

must be continuing to accrete fresh gas from the IGM, which is believed to contain most of the universe's baryons (Fukugita \& Peebles, 2004).

Accreted gas was first predicted to be heated to the virial temperature of the halo before cooling and falling onto the disk (e.g. White \& Frenk, 1991). More recently however, it is thought that in halos at high redshift and low mass halos at low redshift in low density environments, the gas is not heated but instead remains cooler $\left(\mathrm{T} \leq 10^{5} \mathrm{~K}\right)$ as it is accreted (Kereš et al., 2005, 2009). This cold-mode accretion 
should then roughly follow the dark matter filaments associated with the large scale structure of the universe (Dekel \& Birnboim, 2006).

Simulations by Popping et al. (2009) suggest that low column density H i should trace this ongoing accretion. While cold accretion has been tentatively identified in Lyman-limit absorption systems (Shull et al., 2009; Stocke et al., 2010; Ribaudo et al., 2011), detection of this cosmic web in emission is typically not attempted as it requires significant effort to observe with radio telescopes.

\subsection{Diffuse $\mathrm{H}_{\text {I }}$ Discovered in the Local Group of Galaxies}

Braun \& Thilker (2004) reported one of the first possible detections of the cosmic web directly in emission. Using the Westerbork Synthesis Radio Telescope (WSRT), they treated each dish in the array as a separate total power receiver. This allowed them to have several beams mapping the sky simultaneously. Using this method, they conducted a very sensitive $21 \mathrm{~cm}$ survey of the Local Group of galaxies.

The Local Group's largest members include our own Milky Way and M31. M31 is a large spiral galaxy like the Milky Way (Beaton et al., 2007). It is similar in total mass to our Galaxy, $10^{12} \mathrm{M}_{\odot}$ (Evans \& Wilkinson, 2000), at a distance of at least 770 kpc (Brunthaler et al., 2005). About $200 \mathrm{kpc}$ to the southeast is the smaller spiral

galaxy M33 (Brunthaler et al., 2005). There are also a large number of smaller dwarf galaxies. IC10 specifically is a small starbursting galaxy directly to the north of M31.

Braun \& Thilker (2004) discovered several H I features associated with the foreground emission from the Milky Way and also suggested that a significant portion 
of the foreground contamination was due to material from the Magellanic Stream, which we now know to be a correct assumption (Nidever et al., 2010). After filtering out the foreground, they discovered a very diffuse stream of $\mathrm{H}$ I lying directly between the galaxies M31 and M33, as well as diffuse, extended emission to the northwest of M31. The detected column densities in $\mathrm{H}_{\mathrm{I}}$ for the stream did not rise much above $\mathrm{N}_{\mathrm{HI}} \sim 10^{17.5} \mathrm{~cm}^{-2}$. They interpreted this emission to be the neutral component of a condensing intergalactic filament.

A few years after the Westerbork results, Bekki (2008) used test particle simulations to show that a similar stream of material could have been produced if M31 and M33 had some form of tidal interaction roughly 4-8 Gyr ago, where gravitational forces between M31 and M33 could have pulled gas off of one or both galaxies. Not long after this work, the PAndAS survey of red giant branch stars in the Local Group found evidence of tidal features around M33 (McConnachie et al., 2009) along with the many known stellar streams that extend out from M31 (e.g. Ibata et al., 2007).

To try and uncover further details of the H I emission, Lockman et al. (2012) used the Green Bank Telescope (GBT) to conduct a deep mapping of the region between M31 and M33. The GBT beam provides more than a factor a five better spatial resolution than the Westerbork results, but Lockman et al. (2012) were unable to reach the same sensitivities as the Westerbork data. Nevertheless, they did detect emission over a rather scattered area of their maps, indicating that the gas was more sparsely distributed than previously thought. Further deep GBT observations were needed to map and reveal the details of this diffuse gas. 


\subsection{High Velocity and Intermediate Velocity Clouds}

For over 50 years, $\mathrm{H}$ i clouds at velocities that exceed those normally allowed by Galactic rotation have been known (van Woerden et al., 1957; Muller et al., 1963). These anomalous velocity clouds have several proposed origins. The H I could be condensations from the Galactic halo (Spitzer, 1956). They could be primordial gas clouds (Oort, 1966) or tidal debris from surrounding dwarf galaxies (Bland-Hawthorn et al., 1998; Bregman, 2004). The gas could also be material blown out of the Galactic disk that is now falling back toward the plane of the Milky Way (e.g. Bregman, 1980; Shapiro \& Field, 1976). Gas from the halo or outside the galaxy would likely be at higher velocities compared to the disk, while gas from the plane is likely to be found at lower velocities. The origin of the $\mathrm{H} \mathrm{r}$ is likely to be a combination of these possibilities.

Clouds at lower velocities compared to the Milky Way rotation, now known as the intermediate velocity clouds (IVCs), typically have metallicities higher than that seen in the Local Group or intergalactic medium (IGM). The higher metal content suggests that IVCs likely have a Galactic origin. High velocity clouds, or HVCs, have velocities that deviate from the Milky Way by $\sim 90 \mathrm{~km} \mathrm{~s}^{-1}$ or more. HVCs usually have lower metallicity than the IVCs, suggesting an origin outside the Galactic disk.

No large HVC complexes exist in the southern sky, aside from the Magellanic system. Furthermore, no comprehensive map of IVC emission in the southern sky has been published. To help further our understanding of the HVC and IVC phenomena, a better understanding of their distribution across the southern sky is necessary. 
Previous all sky H i surveys have provided high spatial resolution (Barnes et al., 2001;

Putman et al., 2002) or high velocity resolution observations (Kalberla et al., 2005), but not both. The Galactic All Sky Survey (McClure-Griffiths et al., 2009, GASS) provides both high spatial and velocity resolution of $\mathrm{H}_{\mathrm{I}}$ in the southern sky and can help us examine the gas detail.

\subsection{The Baryonic Tully-Fisher Relation}

The Tully-Fisher relation (Tully \& Fisher, 1977) is an empirical relationship between the stellar luminosity $\mathrm{L}$ of a disk galaxy and its rotational velocity $\mathrm{v}_{\text {rot }}$. Specifically, $\mathrm{L} \sim \mathrm{v}_{\mathrm{rot}}^{4}$.

The virial theorem (Clausius, 1870) allows us to relate the rotational speed of a star to the dynamical mass $\mathrm{M}_{\mathrm{dyn}}(\mathrm{r})$ of the galaxy interior to its orbit of radius $\mathrm{r}$

$$
\mathrm{v}_{\mathrm{rot}}^{2} \sim \frac{\mathrm{M}_{\mathrm{dyn}}(\mathrm{r})}{\mathrm{r}}
$$

If one assumes that the mass and luminosity are related by some constant mass-tolight ratio $\Upsilon=\frac{\mathrm{M}_{\mathrm{dyn}}}{\mathrm{L}}$, then we have that

$$
\mathrm{v}_{\mathrm{rot}}^{2} \sim \frac{\Upsilon \mathrm{L}}{\mathrm{r}} \sim \frac{\mathrm{L}}{\mathrm{r}}
$$

The luminosity of a galaxy is related to its surface brightness I

$$
\mathrm{L}=4 \pi \mathrm{r}^{2} \mathrm{I} \sim \mathrm{r}^{2} \mathrm{I},
$$


so that we have, assuming the surface brightness is constant

$$
\begin{gathered}
\mathrm{v}_{\text {rot }}^{2} \sim \frac{\mathrm{L}}{\mathrm{r}} \sim \mathrm{L}\left(\frac{\mathrm{I}}{\mathrm{L}}\right)^{\frac{1}{2}} \sim \mathrm{L}^{\frac{1}{2}} \\
\mathrm{v}_{\text {rot }}^{4} \sim \mathrm{L} .
\end{gathered}
$$

Why the luminous matter, the source of $\mathrm{L}$, is related to the dynamical mass, which traces both luminous and dark matter, is uncertain.

The rotational speed of the galaxy can be measured by determining its rotation curve from the velocity field, if one is available. If the galaxy is unresolved by the telescope beam, then the width of the galaxy's spectral profile can be measured to give an estimate of the rotation.

McGaugh et al. (2000) show that by including all baryonic matter, not just luminous matter, a similar Tully-Fisher like relationship exists between the baryonic mass and rotation speed. Specifically, the baryonic Tully-Fisher states that $\mathrm{M}_{\text {baryonic }} \sim \mathrm{v}_{\text {rot }}^{4}$. This is a very tight correlation, with only a small spread in the distribution of galaxies along the relation. This lead McGaugh et al. (2000) to suggest that $\mathrm{M}_{\text {baryonic }}$ is a more fundamental quantity to use as opposed to luminosity.

\subsection{Thesis Outline}

In Chapter 2, we discuss our GBT observations of the faint $\mathrm{H}$ i emission between the galaxies M31 and M33 down to column density limits of $\mathrm{N}_{\mathrm{HI}} \sim 10^{17.2} \mathrm{~cm}^{-2}$. We also discuss our reduction techniques and issues involving the GBT Spectrometer. 
In Chapter 3, we discuss the results from 250 hours of our 400 hour survey to map a 12 square degree region between M31 and M33 to attempt to uncover the origins of the diffuse $\mathrm{H}$.

In Chapter 4, we present and discuss further results using the full 400 hours of data. We briefly mention ongoing work to map other regions around M31. We also present and discuss results from deep pointings conducted with the GBT to the northwest of M31 to search for the diffuse gas that is seen in the Braun \& Thilker (2004) map.

In Chapter 5, we present the most detailed maps of both HVC and IVC gas for the southern sky to date. We examine the basic global properties of this material and the features that are seen.

We finish in Chapter 6 with conclusions and plans for future work on these topics. Appendices A, B and C go into further detail of the fundamental concepts behind $\mathrm{H}_{\mathrm{r}}$ emission and spectral line astronomy. 


\section{Chapter 2}

\section{Mapping Diffuse Neutral Hydrogen with the GBT ${ }^{1}$}

Observations of the gas that resides between galaxies is essential to understanding how large spirals like our Milky Way acquire material for the formation of new stars. The Robert C. Byrd Green Bank Radio Telescope (GBT) has the capability to map the neutral component of this gas directly in emission. This work details the process we have developed to observe extremely faint $21 \mathrm{~cm}$ radiation, in which we reach spectral noise limits of $3 \sigma_{\mathrm{T}_{\mathrm{B}}} \approx 2.6 \mathrm{mK}$ with $\Delta v \approx 5 \mathrm{~km} \mathrm{~s}^{-1}$, where $\sigma_{\mathrm{T}_{\mathrm{B}}}$ and $\Delta v$ are the root-mean-square spectral noise and spectral line width respectively. We provide an explicit discussion of our observing techniques and reduction process, such as the stability of calibration temperatures $\mathrm{T}_{\mathrm{CAL}}$ over several months, as well as a blanking issue inherent to the GBT Spectrometer.

\subsection{Mapping $\mathrm{H}_{\mathrm{I}}$ in the Cosmic Web}

\subsubsection{Background}

To further our understanding of how galaxies acquire fresh gas, we must investigate the diffuse material that resides in filamentary structures between galaxies. The main issue with observing such gas is that it is expected to be mostly ionized (Maloney, 1993), with perhaps 1\% of the gas in the neutral state (Dove \& Shull, 1994),

\footnotetext{
${ }^{1}$ This work will soon be submitted as a GBT memo.
} 
appearing at $\mathrm{H}$ I column densities of $\mathrm{N}_{\mathrm{HI}} \sim 10^{17} \mathrm{~cm}^{-2}$. Thus, it takes significant sensitivity for a radio telescope to observe such emission. One must generally resort to UV absorption line measurements instead, but these require a bright background source for such observations to be possible.

In 2004, extremely faint H i emission in the Local Group between our neighboring spiral galaxies M31 (Andromeda) and M33 (Triangulum) was detected (Braun \& Thilker, 2004). In that work, a column density sensitivity of $\mathrm{N}_{\mathrm{HI}} \sim 10^{17} \mathrm{~cm}^{-2}$ was reached by using every dish in the Westerbork Synthesis Radio Telescope (WSRT) as a single receiver. After thousands of hours and extensive smoothing of the data to $49^{\prime}$ resolution and $18 \mathrm{~km} \mathrm{~s}^{-1}$ velocity resolution, a very faint, extended $\mathrm{H}$ i feature between M31 and M33 was discovered (Figure 2.1). Follow-up observations (Lockman et al., 2012) of the region with the GBT have confirmed the existence of some of this gas, but those observations didn't reach the same sensitivity as the WSRT data.

\subsubsection{GBT Mapping Sensitivity}

Reaching $21 \mathrm{~cm}$ sensitivities of $\sim 10^{17} \mathrm{~cm}^{-2}$ with no spatial smoothing using the GBT is feasible. The radiometer equation tells us

$$
\sigma_{\mathrm{T}_{\mathrm{B}}}=\frac{\mathrm{K} 1 \cdot \mathrm{T}_{\mathrm{sys}}}{\sqrt{\mathrm{K} 2 \cdot \mathrm{N}_{\mathrm{pol}} \cdot \Delta \nu \cdot \mathrm{t}_{\mathrm{eff}}}}
$$

where $\sigma_{\mathrm{T}_{\mathrm{B}}}$ is the predicted root-mean-square (RMS) of the brightness temperature, or antenna temperature of a typical spectrum over one GBT beam. $\mathrm{T}_{\text {sys }}$ is the system

temperature, around $18-20 \mathrm{~K} . \mathrm{N}_{\text {pol }}$ is the number of polarizations, usually $2 . \Delta \nu$ 


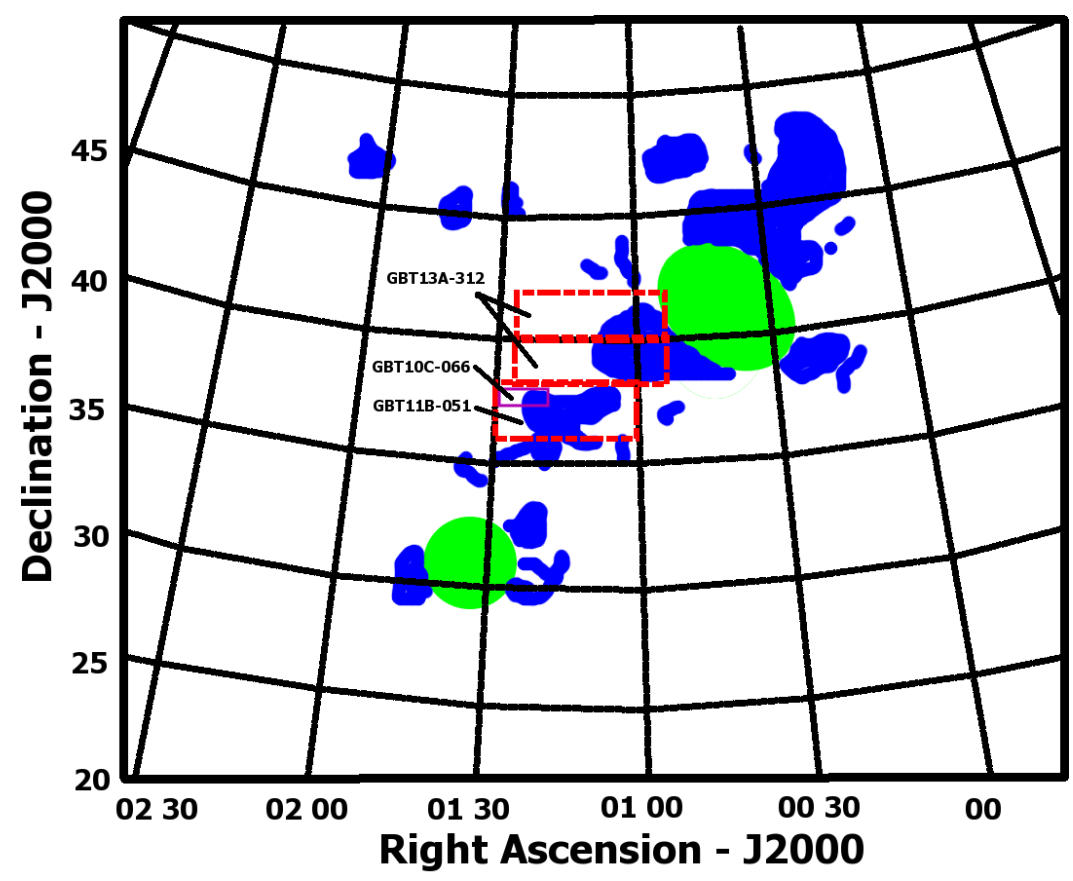

Figure 2.1: An approximate figure representing an area of the Local Group that was observed by the WSRT (Braun \& Thilker, 2004). The green areas roughly represent the primary HI emission from the galaxies M31 and M33, while the blue is the extended emission seen about both galaxies. The dashed boxes are areas we have mapped with the GBT (GBT11B-051, GBT13A-312). The small purple outline is the area we mapped as an exploratory project (GBT10C-066).

is the bandwidth per unit spectral frequency channel in $\mathrm{Hz}$ and $\mathrm{t}_{\text {eff }}$ is the effective integration time of the observation in seconds. K1 and K2 are correction factors specific to the GBT Spectrometer; specifically, $\mathrm{K} 1=1.032$ and $\mathrm{K} 2=1.21$. So, the radiometer equation becomes, using $\mathrm{T}_{\mathrm{sys}}=18 \mathrm{~K}$ and $\Delta \nu=1.52 \mathrm{kHz}$

$$
\sigma_{\mathrm{T}_{\mathrm{B}}}=\frac{306 \mathrm{mK}}{\sqrt{\mathrm{t}_{\mathrm{eff}}}}
$$

H I column density is determined from the brightness temperature integrated over the spectral line in frequency (or velocity). Assuming that the $\mathrm{H}$ i is optically thin (see Appendix B) 


$$
\mathrm{N}_{\mathrm{HI}}=1.822 \times 10^{18} \int_{\text {line }}\left(\frac{\mathrm{T}_{\mathrm{B}}(\mathrm{v})}{\mathrm{K}}\right)\left(\frac{\mathrm{dv}}{\mathrm{km} \mathrm{s}^{-1}}\right) \mathrm{cm}^{-2}
$$

If the spectral noise is gaussian in nature, we can write the error in column density as

$$
\sigma_{\mathrm{N}_{\mathrm{HI}}}=1.822 \times 10^{18} \cdot \sigma_{\mathrm{T}_{\mathrm{B}}} \cdot \sqrt{\mathrm{W} \cdot \Delta \mathrm{v}} \mathrm{cm}^{-2}
$$

where $\mathrm{W}$ is the linewidth of the $\mathrm{H}$ i spectrum and $\Delta \mathrm{v}$ is the width of a spectral channel. Both are in units of $\mathrm{km} \mathrm{s}^{-1}$. A $1.52 \mathrm{kHz}$ channel width converts to $0.32 \mathrm{~km} \mathrm{~s}^{-1}$. Assuming a typical $\mathrm{H}_{\mathrm{I}}$ linewidth of $25 \mathrm{~km} \mathrm{~s}^{-1}$, a $3 \sigma \mathrm{H}$ I column density detection limit of $10^{17} \mathrm{~cm}^{-2}$ would require, inverting Eq. 2.4, $\sigma_{\mathrm{T}_{\mathrm{B}}}=6.5 \mathrm{mK}$. Inserting this result into Eq. 2.2 and solving gives an effective integration time of $37.2 \mathrm{~min}$ per GBT beam.

Given that the main GBT beam is about $9.1^{\prime}$ across, there are roughly 44 beams (43.5) per square degree. Thus, to map $21 \mathrm{~cm}$ radiation down to $10^{17} \mathrm{~cm}^{-2}$ requires an effective time of about 27 hours per square degree. While this may seem like a significant amount of time, with careful planning of the observations, one can still map rather sizeable regions.

Furthermore, the unblocked aperture design (Figure 2.2) significantly reduces the amount of contamination by stray radiation, with the side-lobes of the beam being roughly $30 \mathrm{~dB}$ below that of the main beam. This, plus the stability of the receivers and low system temperature also ensure that the telescope can consistently map faint H I emission. 


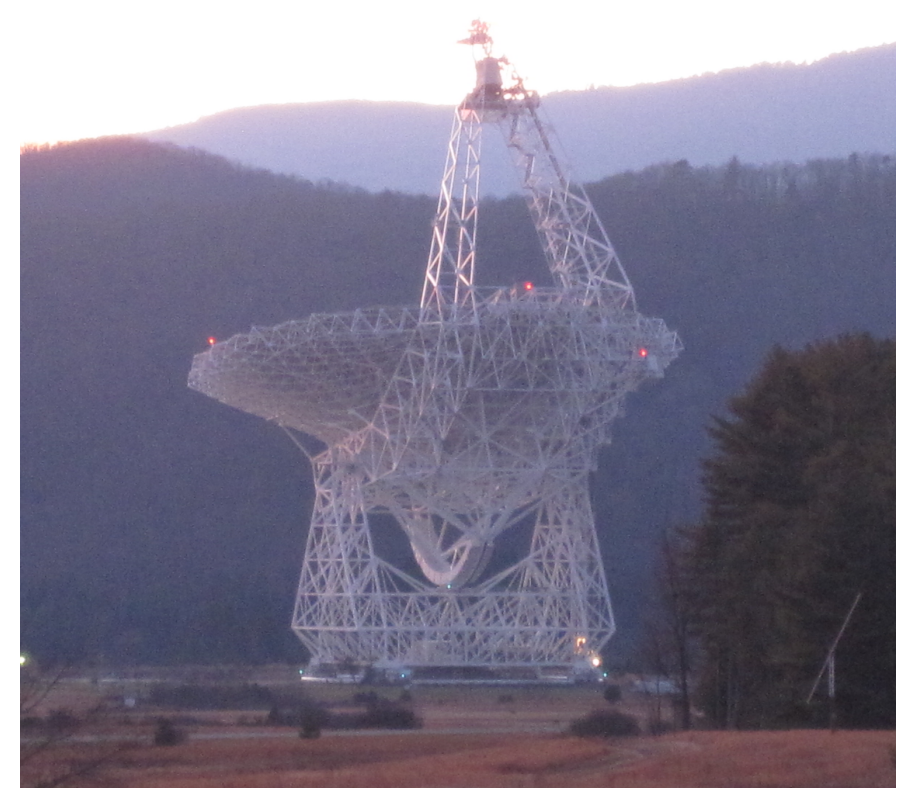

Figure 2.2: The GBT. Image taken by the author.

\subsection{Observing Method}

\subsubsection{Concept}

A major issue with observing extremely faint emission is ensuring that there is little to no baseline structure in the spectral data. Frequency switching can produce baseline artifacts that could be potentially confused with real emission features. Position switching can eliminate many of these structures by providing more stable baselines, but the telescope must generally slew to a different part of the sky to obtain off-source spectra for reference. See Appendix C. For a project requiring hundreds of hours of observations, the accumulated time slewing the telescope can greatly increase the amount of observing time needed. 


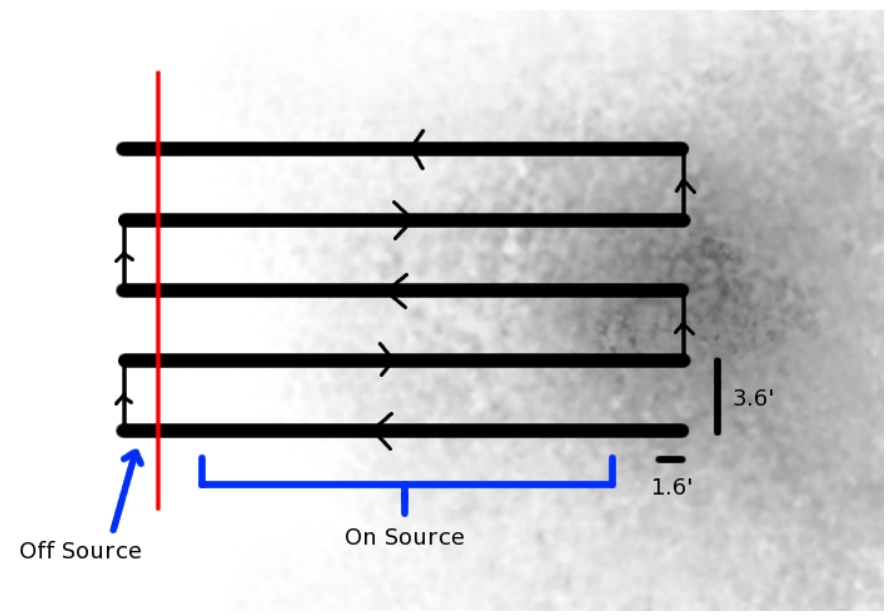

Figure 2.3: An illustration of the observing method. We scan in right ascension, recording data at $1.6^{\prime}$ intervals. We then jump in declination to the next row by $3.6^{\prime}$. Our map is extended to the east to include areas believed to be free of emission.

For our projects (GBT10C-066, GBT11B-051, GBT13A-312), we decided to choose a region of our proposed mapping area to use as our reference positions. Specifically, we used the WSRT map and determined that the eastern edge of our observing areas would be suitable to use for reference positions (Figure 2.1). This gave us the advantages of having a reference for each scan, allowing us to choose the total number of integrations that can be used as a reference and allowed us to devote the majority of our observing time to actually mapping the region without needing to slew the telescope off source. See Figure 2.3 for a simple illustration. One must take careful note, however, that the area of our scans chosen for the references must contain no emission (at least down to the desired column density sensitivity).

\subsubsection{Front End and Back End Configuration}

The data were collected in total power mode, which simply collects photons as the telescope is scanned, since the actual reference selection and subtraction would 
be done during the reduction. We used the L-band receiver $(1.10-1.75 \mathrm{GHz})$ and GBT Spectrometer with a $12.5 \mathrm{MHz}$ bandwidth using 8192 channels centered near the $1420 \mathrm{MHz}$ line, giving $\Delta \nu=1.52 \mathrm{kHz}$. We also tried using a $50 \mathrm{MHz}$ bandwidth and four different frequency windows (IFs), with the other three centered on the $\mathrm{OH}$ lines $(1612,1665,1667,1720 \mathrm{MHz})$. We found, however, that strong radio frequency interference (RFI) in the $1612 \mathrm{MHz}$ window caused large swings in the power levels of the other IFs. Thus, we focused solely on the $1420 \mathrm{MHz}$ window and shrunk the bandwidth to $12.5 \mathrm{MHz}$.

\subsubsection{Observing the Calibrator 3C48}

To ensure the spectra were properly calibrated, that is, converted from raw

counts to antenna temperature, we first observed a nearby flux calibrator 3C48 before the $\mathrm{H}$ I mapping. We conducted pointing scans on 3C48, which tests the main beam response of the telescope. An example of the pointing is shown in Figure 2.4. Then we conducted an "OffOn" position switching with each position lasting 60 seconds. An example of the raw spectra is shown in Figure 2.5.

\subsubsection{Mapping the $\mathrm{H}_{\mathrm{I}}$}

Our maps are $6^{\circ} \times 2^{\circ}$ in size. We chose to perform a raster scan in right ascension, sampling for three seconds every $1.6^{\prime}$. We then jumped in declination to the next scan row by $3.6^{\prime}$. This ensures that our area is Nyquist sampled so that no spatial information in our maps is lost. There are 34 rows in all, with each row taking 

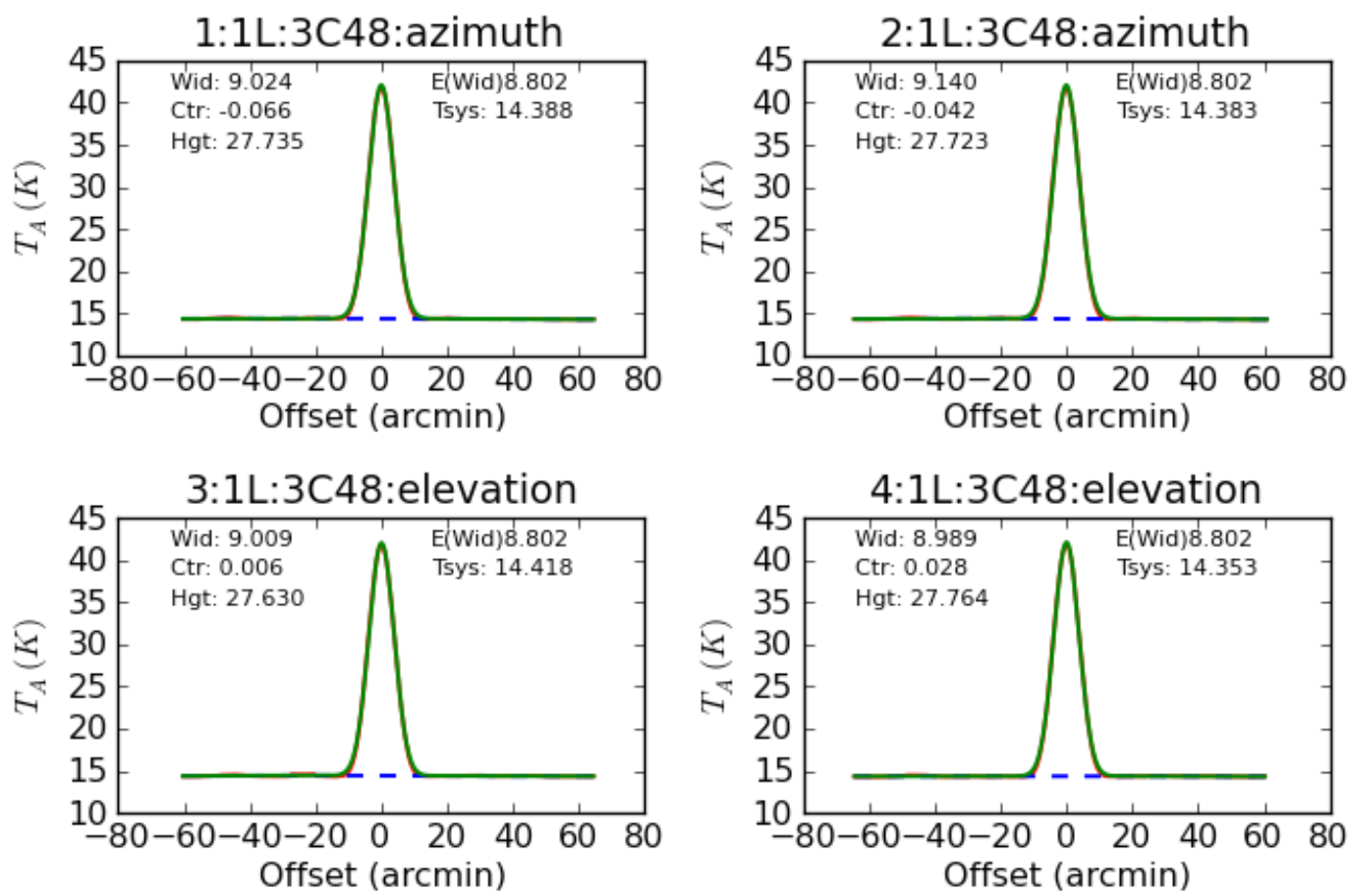

Figure 2.4: An example of a GBT pointing on 3C48, as seen in ASTRID, to examine the main beam response of the telescope. Here, the beamwidth is correct $\left(\sim 9^{\prime}\right)$ and the gaussian height corresponds to the known brightness of $3 \mathrm{C} 48$ (in K).

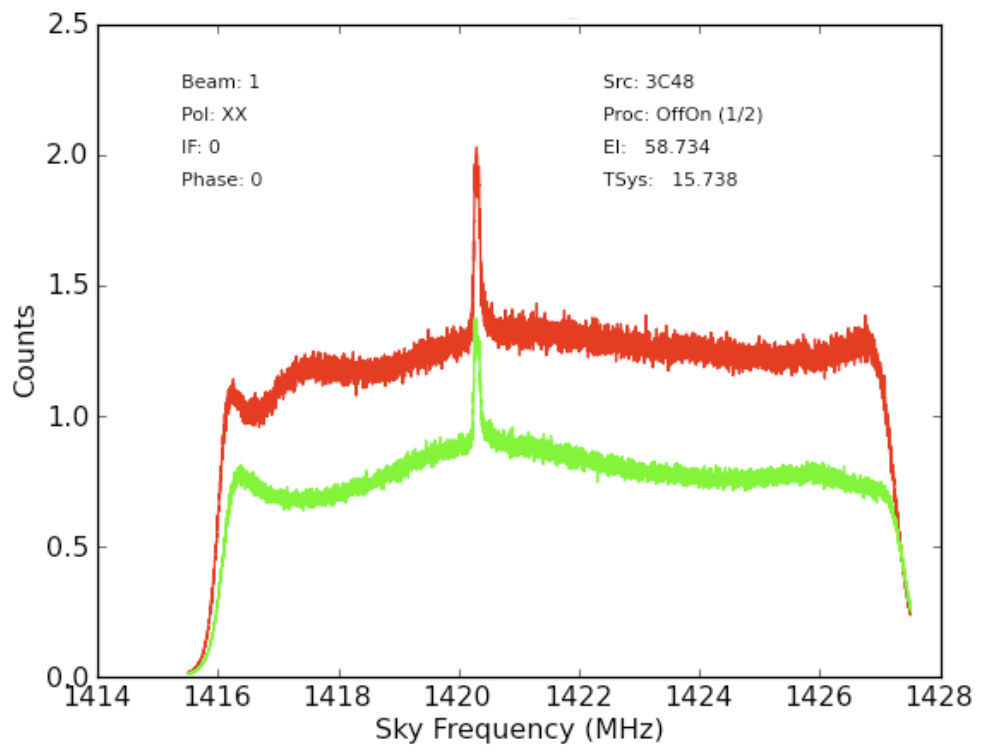

Figure 2.5: Reference spectra from $3 \mathrm{C} 48$ as seen in ASTRID. The red spectrum is for the $\mathrm{X}$ polarization and green is for the Y polarization. 


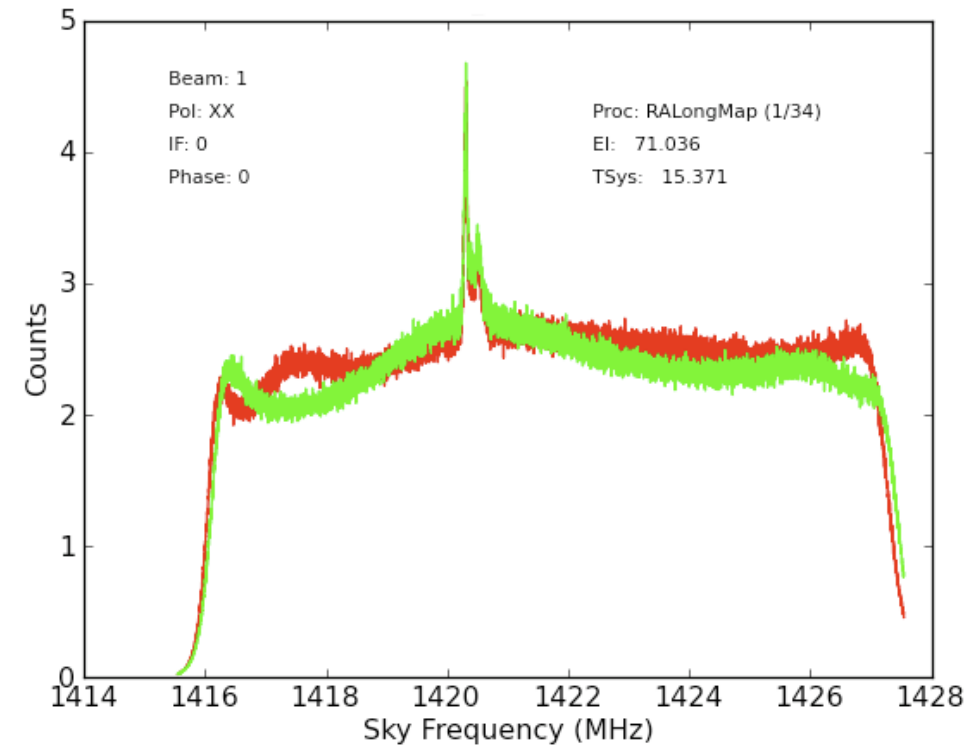

Figure 2.6: The spectra in both polarizations, here $\mathrm{X}$ and $\mathrm{Y}$, from one three second integration of the H I mapping scans.

11.25 minutes to complete. We observed the same area repeatedly until we reached the desired sensitivity, which took 400 hours of time. An example spectrum from one three-second integration is shown in Figure 2.6.

\subsection{Data Reduction}

\subsubsection{Concept}

To remove the bandpass structure in spectral data, one must subtract the reference source spectra from the on source spectra, which we will call the signal, so that only the spectra of the desired source remains. In terms of temperature

$$
\mathrm{T}_{\mathrm{src}}=\mathrm{T}_{\mathrm{CAL}} \cdot \frac{\mathrm{P}_{\mathrm{sig}}-<\mathrm{P}_{\mathrm{ref}}>}{<\mathrm{P}_{\mathrm{CALon}}-\mathrm{P}_{\mathrm{CALoff}}>_{\mathrm{ref}}} .
$$


See Appendix C for a more detailed discussion.

$\mathrm{T}_{\mathrm{CAL}}$ can be determined from observations of a flux calibrator as described in the following section. $\mathrm{P}_{\text {sig }}$ and $\mathrm{P}_{\text {ref }}$ are the input power levels, in counts, and $\mathrm{P}_{\mathrm{CALon}}$ and $\mathrm{P}_{\text {CALoff }}$ are the power levels when the front end noise diode is on and off respectively. Since we use the eastern most integrations of our map as the reference, we average them for Equation 2.5. The reduction of the raw data was done in GBTIDL ${ }^{2}$.

\subsection{2 $\mathrm{T}_{\mathrm{CAL}}$ from $3 \mathrm{C} 48$}

To determine the $\mathrm{T}_{\mathrm{CAL}}$ values for a given observation, we used the "OffOn" observations of the flux calibrator 3C48. We first loaded the data from a particular observing session into a working directory by using the sdfits command.

We then took the $3 \mathrm{C} 48$ OffOn scans and calculated the $\mathrm{T}_{\mathrm{CAL}}$ values using scal.pro, contributed by Ron Maddalena ${ }^{3}$. The inputs of relevance here are for the opacity and aperture efficiency, namely tau $=[0.01]$ and ap_eff $=[0.657543]$. See Appendix $\mathrm{C}$ for more information.

The $\mathrm{T}_{\mathrm{CAL}}$ values obtained from our observations were remarkably stable, even over a period of several months, with values of $1.56 \pm 0.05(1 \sigma)$ for both polarizations. A histogram of the $\mathrm{T}_{\mathrm{CAL}}$ values over 103 observing sessions spanning 11 months is shown in Figure 2.7.

The $\mathrm{T}_{\mathrm{CAL}}$ values for each polarization were then applied to the reduced spectra (Equation 2.5) for that particular observing session. The stability of $\mathrm{T}_{\mathrm{CAL}}$ over such

\footnotetext{
${ }^{2}$ http://gbtidl.nrao.edu/

${ }^{3}$ http://www.gb.nrao.edu/ rmaddale/
} 


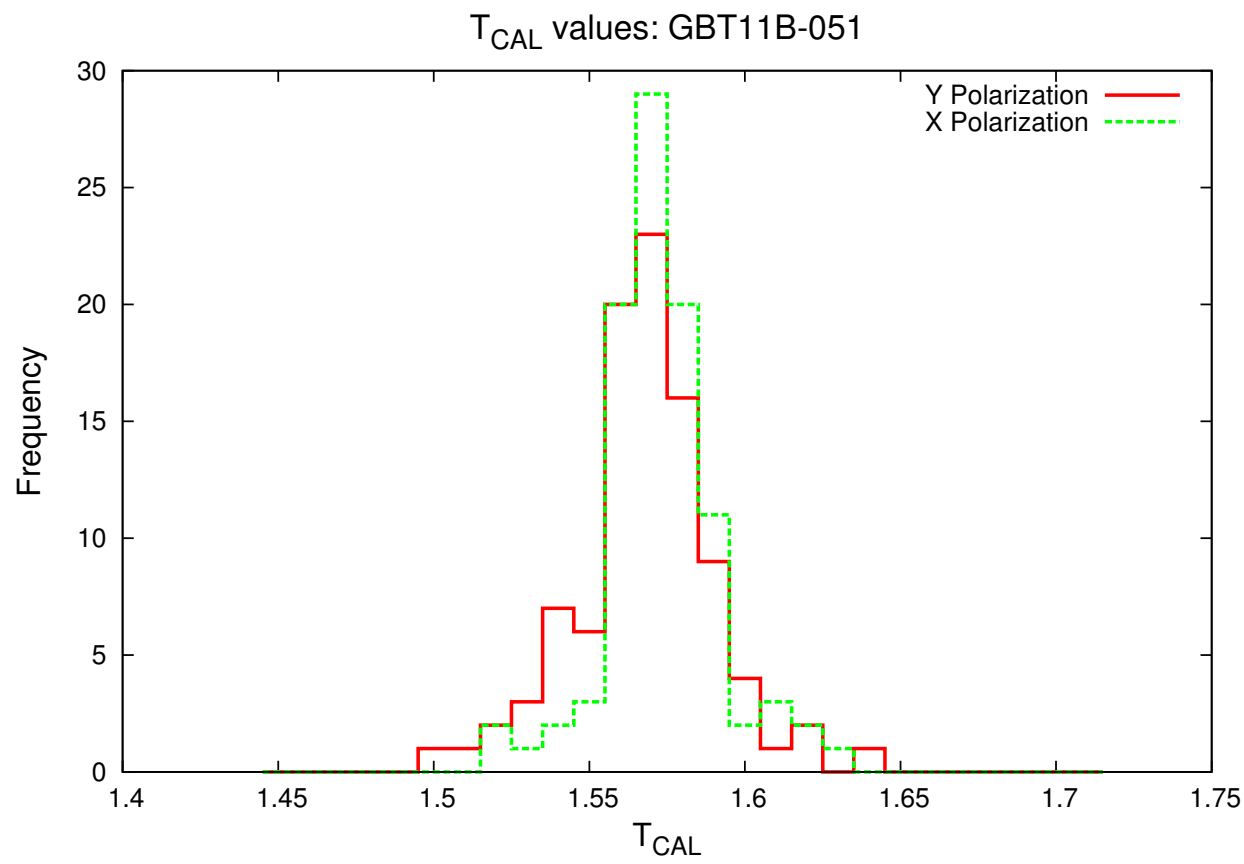

Figure 2.7: A histogram of calculated $\mathrm{T}_{\mathrm{CAL}}$ values, 206 in all, for both polarizations, over a period of 11 months.

an extended period is quite significant. For instance, if the observation of a flux calibrator is not possible for a particular observing session, one can use the inferred value of $\mathrm{T}_{\mathrm{CAL}}$ from recent observations with a likely error of only a few percent.

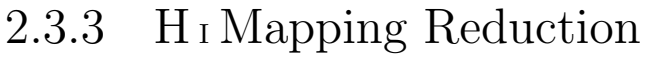

Each telescope scan in right ascension is defined by a sequence number, in our case, ranging from 1 (the first row) to the 34 (the last row). For our observations, odd sequences scanned from west to east and even numbers east to west. Thus, to select the eastern most integrations per scan, we first called a scan with gettp to extract the sequence number and used a modulo 2 argument to determine whether to use the first few or last few integrations to average for the reference. 
Once we determined which integrations to use for the reference, we then extracted the CALon and CALoff phases separately to take the difference. We did this with the get command. We took the difference between the on and off phase and averaged over all 10 reference integrations to get $\left.<\mathrm{P}_{\text {CALon }}-\mathrm{P}_{\text {CALoff }}\right\rangle_{\text {ref }}$. We then collected the reference integrations with select and averaged to get $\left.<\mathrm{P}_{\text {ref }}\right\rangle$. Then we used gettp to collect each signal integration $\left(\mathrm{P}_{\text {sig }}\right)$ to compute the ratio in Equation 2.5 and then multiplied by $\mathrm{T}_{\mathrm{CAL}}$.

We also corrected for atmospheric effects. We did this by multiplying a factor that is dependent upon the telescope's elevation at the time the spectra were taken. One can retrieve the telescope elevation, in degrees, with the !g structure, scaling the reduced spectra by $\mathrm{e}^{\frac{\tau}{\sin (\mathrm{el})}}$ where $\tau$ is the opacity of the atmosphere at zenith and el is the elevation of the integration.

We then fit a second order baseline to the spectra to ensure any remaining large scale baseline structure was removed. The region chosen for fitting did not include frequency ranges believed to contain any $\mathrm{H}$ i emission.

After the baseline was subtracted, we then computed the RMS of the spectra over an emission free region. If the RMS was less than three times the expected value from the radiometer equation, we continued, otherwise we discarded the spectrum. This ensured that most spectra with baseline structure, mainly due to RFI and instrumental effects, were removed. Only a small fraction of the reduced spectra $(\sim 4 \%)$ had significant enough baseline structure to be discarded. The remaining spectra were then truncated to the desired frequency range using dcextract. Finally, the data were smoothed from $0.3 \mathrm{~km} \mathrm{~s}^{-1}$ to $5 \mathrm{~km} \mathrm{~s}^{-1}$. We did this using a 16 channel boxcar 

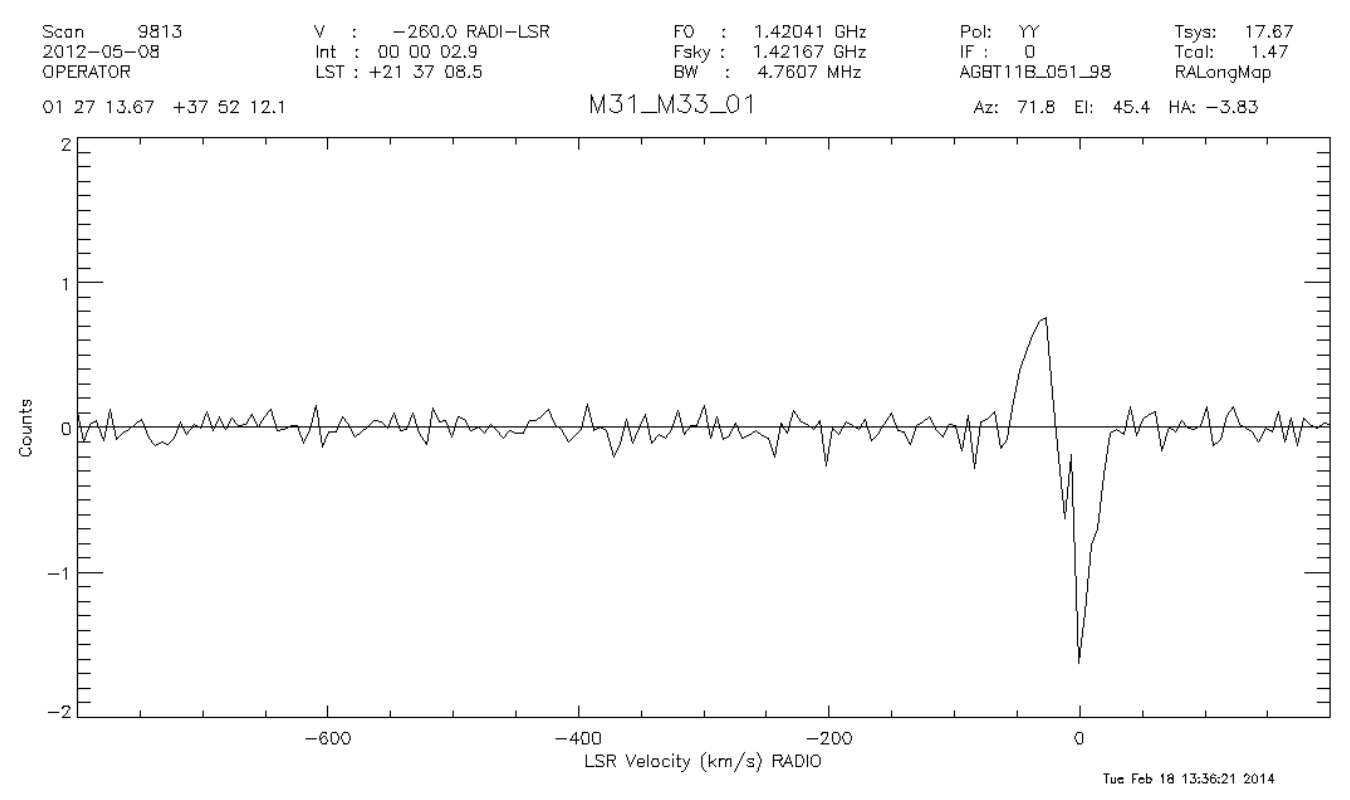

Figure 2.8: A reduced H I spectrum. The emission of interest lies at or near $-260 \mathrm{~km} \mathrm{~s}^{-1}$, but one integration is not enough for any spectrum to be visible. The large swings near $0 \mathrm{~km} \mathrm{~s}^{-1}$ are due to incomplete subtraction of the Milky Way H I.

smoothing and removed the redundant channels. We then saved the spectrum. This process was carried out for each polarization. An example of the reduced spectra is shown in Figure 2.8.

We measured the RMS noise of the reduced spectra, from -800 to $-260 \mathrm{~km} \mathrm{~s}^{-1}$ and also computed the expected RMS noise, using the radiometer equation. The effective integration time, using 10 integrations for the reference, and 2.9s for the signal time, we have

$$
\mathrm{t}_{\text {effective }}=\frac{\mathrm{t}_{\mathrm{sig}} \cdot \mathrm{t}_{\mathrm{ref}}}{\mathrm{t}_{\mathrm{sig}}+\mathrm{t}_{\mathrm{ref}}}=\frac{10}{11} \cdot \mathrm{t}_{\mathrm{sig}}=\frac{10}{11} \cdot 2.9 \mathrm{~s}=2.6 \mathrm{~s}
$$

Smoothing the original resolution, $1.52 \mathrm{kHz}$ by a factor of 16 gives

$$
\Delta \nu=\frac{12.5 \mathrm{MHz}}{8192} \cdot 16=24.4 \mathrm{kHz}
$$




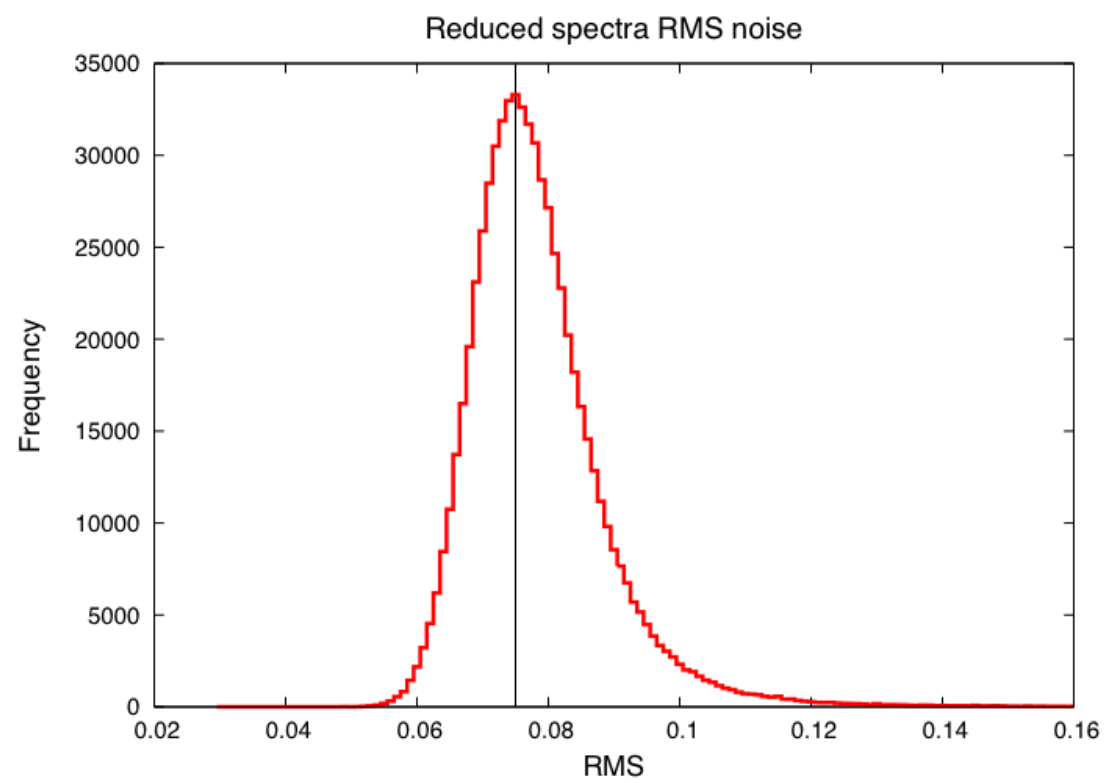

Figure 2.9: A histogram of the RMS noise of the reduced H I spectra over all observing sessions. The vertical line represents the expected RMS noise value of $74 \mathrm{mK}$ from Eq. 2.9.

which is equivalent to $5.2 \mathrm{~km} \mathrm{~s}^{-1}$. We also measured the values of $\mathrm{T}_{\text {sys }}$ over several scans using

$$
\mathrm{T}_{\mathrm{sys}}=\mathrm{T}_{\mathrm{CAL}} \cdot \frac{<\mathrm{P}_{\text {ref }}>}{<\mathrm{P}_{\mathrm{CALon}}-\mathrm{P}_{\mathrm{CALoff}}>_{\mathrm{ref}}} \approx 20 \mathrm{~K}
$$

Using this for $\mathrm{T}_{\text {sys }}$, along with $\mathrm{K} 1=1.032$ and $\mathrm{K} 2=1.21$ gives

$$
\sigma_{\mathrm{T}}=\frac{1.032 \cdot 20 \mathrm{~K}}{\sqrt{1.21\left(\frac{12.5 \mathrm{MHz}}{8192} \cdot 16\right)\left(2.9 \mathrm{~s} \cdot \frac{10}{11}\right)}}=74 \mathrm{mK}
$$

From the RMS noise measurements, we found that $\sigma_{\mathrm{T}}=(75 \pm 10) \mathrm{mK}$, which is in very good agreement with the predicted value (Figure 2.9). The $10 \mathrm{mK}$ spread is primarily due to observations at low elevation, which increase the system temperature and thus the noise. 


\subsection{Blanking Issues with the Spectrometer}

Upon inspection of our exploratory data (GBT10C-066), we discovered that the system temperatures were increasing as we scanned across our map. This was always an increase, regardless of the scan direction, indicating that this was not an effect due to the sky. The drifts were severe enough, around $2 \mathrm{~K}$, to make the initial data unusable.

This effect was primarily due to Spectrometer blanking. There are two types of blanking. When the noise diode switches phases, from CALoff to CALon and vice versa, the data are blanked during the transition. This is "internal blanking". "External blanking", or Doppler blanking, occurs when the reference frequency is shifted to account for Doppler shifts due to tracking across the sky and occurs at indefinite times. In our case, the external blanking was occurring during every CALoff phase and was slowly drifting into the CALon phase across each scan. As a result, the exposure times, the total integration duration minus blanking, were changing across the scan and thus causing the system temperatures to appear to drift. See Figure 2.10 for an example of this.

If we define $t_{\text {duration }}$ as the total time of an integration, and $t_{\text {internal }}$ and $t_{\text {external }}$ as the amount of time the data were blanked by internal and external blanking respectively, then the Spectrometer should, in principle, correct blanking by scaling the power levels by

$$
\mathrm{P}_{\text {scaled }}=\mathrm{P} \cdot \frac{1}{\mathrm{t}_{\text {duration }}-\left(\mathrm{t}_{\text {internal }} \cup \mathrm{t}_{\text {external }}\right)}
$$




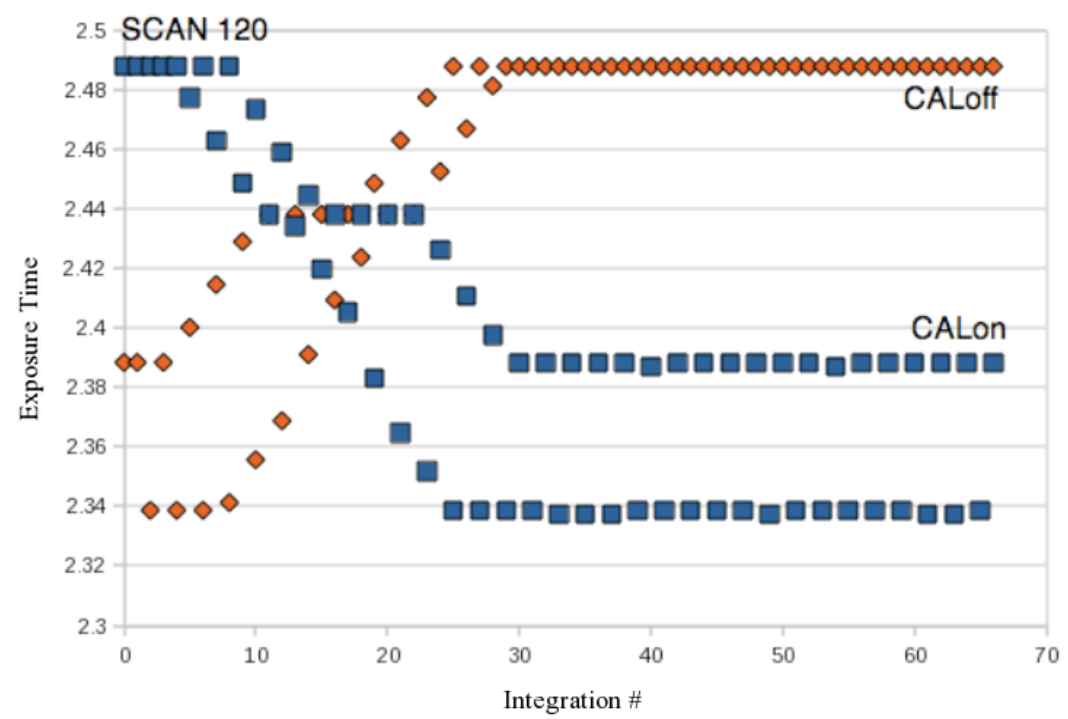

Figure 2.10: The effect of Doppler blanking on the exposure times of both the CALon and CALoff phases. The rapid oscillations are because the blanking happens every other CALoff phase and then drifts to the CALon phase across the scan.

The data however, are actually scaled by

$$
\mathrm{P}_{\text {scaled }}=\mathrm{P} \cdot \frac{1}{\mathrm{t}_{\text {duration }}-\max \left(\mathrm{t}_{\text {internal }} \text { OR } \mathrm{t}_{\text {external }}\right)}
$$

depending upon whether $t_{\text {internal }}$ or $t_{\text {external }}$ is larger.

This caused the apparent drift in system temperature. It should be noted that this drift is not an issue with frequency switched data, given that blanking occurs automatically before each integration, preventing the drift. Tracking a source for position switching also reduces the effect since one is not scanning on the sky. Furthermore, the closer one observes on the sky to the vernal equinox point, the more corrections that are required, enhancing the effect. We have confirmed that the effect is present in other data sets, as shown in Figure 2.11. 


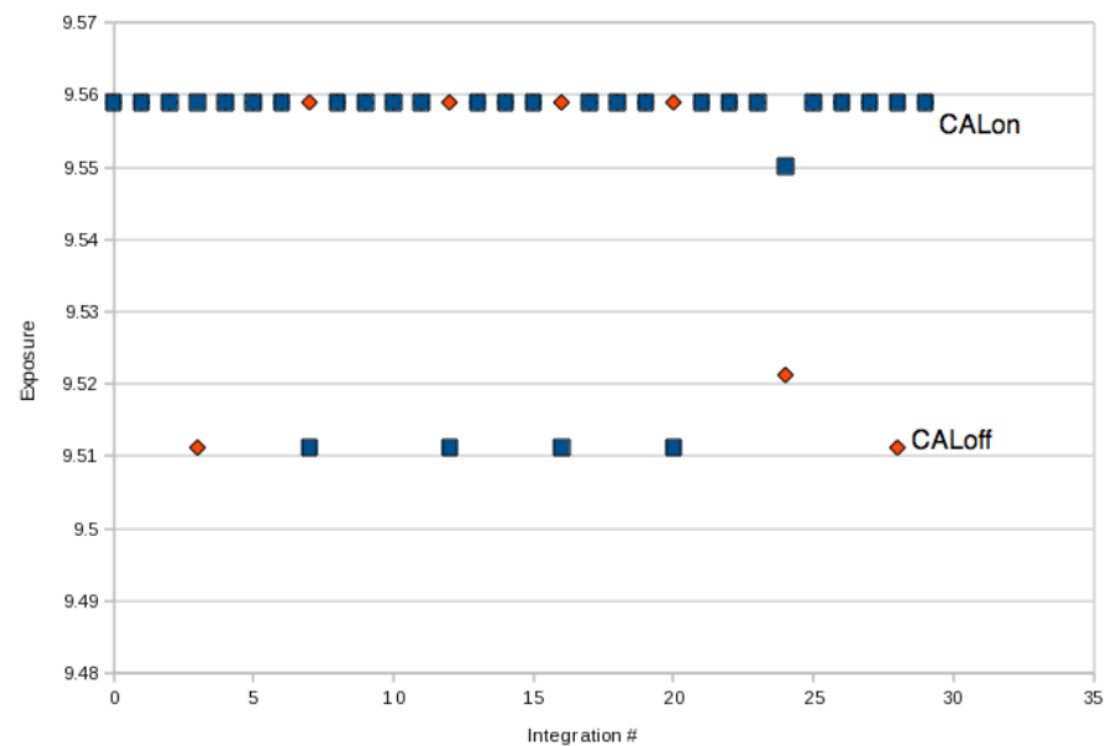

Figure 2.11: Exposure times taken from a tracking scan of GBT10A-043. The effect of drift is present, although not as severe. Data provided by Jay Lockman.

To prevent this issue from occurring, we added to our observing scripts the following, after configuration and before the telescope slew

SetValues( "LO1",\{ "tolerance":1000000\})

SetValues("LO1",\{ "state": "prepare" \}),

This increases the amount of allowed, yet still small, drift in frequency before Doppler tracking occurs and corrected the problem of system temperature drift in our scans.

\subsection{Gridding the Spectral Line Data}

To make maps of the emission, one must grid the spectral line data into a data cube. We used the Astronomical Image Processing System, or AIPS ${ }^{4}$. First, the data were converted from their IDL format to an sdfits format that AIPS could utilize. This was accomplished by using idlToSdfits, written by Glen Langston. It is

\footnotetext{
${ }^{4}$ http://www.aips.nrao.edu/index.shtml
} 
important to note that data blocks with repeating scan numbers cannot be used in idlToSdfits, or else the data with repeated scan numbers will not be converted. The repeating scans must be loaded separately.

After the data were converted into sdfits, each block of data was then loaded into AIPS using the task UVLOD. Each reduced data block had to be concatenated together for the gridding. The task DBCON accomplished this.

Once the data were concatenated together, we used the task SDGRD for the gridding. Here, one can use inputs that are typical for H I mapping with the GBT (Mangum et al., 2007), such as:

CELLSIZE 210

BCHAN 0

ECHAN 0

XTYPE -16

YTYPE -16

XPARM 5502754622

YPARM 5502754622

which convolves the data with an exponentially truncated, circular Bessel function. Other types of functions can be used via the XTYPE, YTYPE inputs and the corresponding XPARM, YPARM parameters, depending upon the users preference. One can review the AIPS SDGRD help page ${ }^{5}$ for further information.

Once the data are gridded into a cube, the standard analyses can be performed, such as creating moment maps with the TRANS and MOMNT tasks. It is also advis-

\footnotetext{
${ }^{5}$ http://www.aips.nrao.edu/cgi-bin/ZXHLP2.PL?SDGRD
} 
able that one subtract out any remaining baseline structure using the task XBASL.

An example moment-zero map of our current survey data (GBT11B-051, GBT13A-

312) is shown in Figure 2.12.

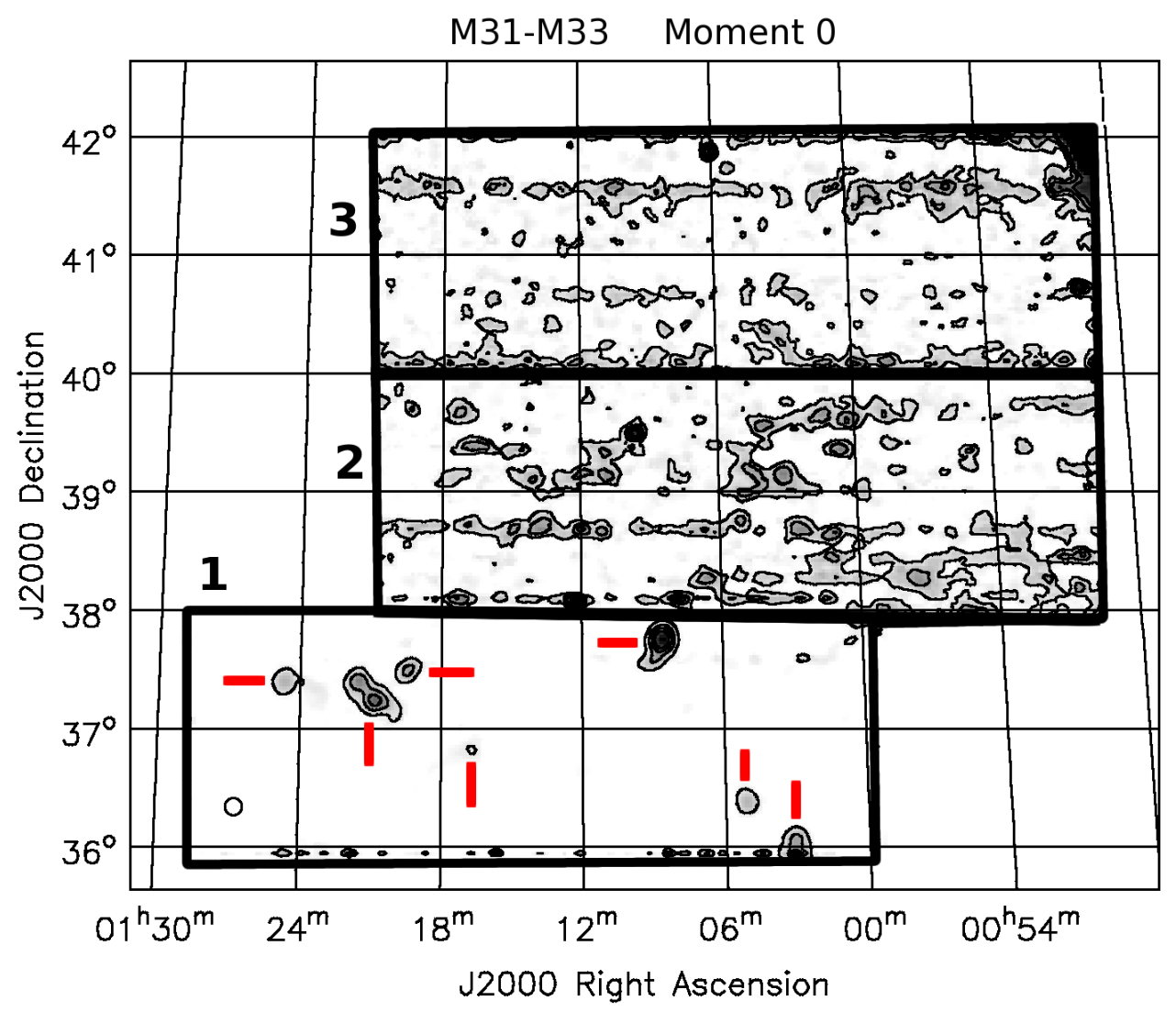

Figure 2.12: A moment zero map of our H I survey data. Region 1 is for 400 hours of observations (GBT11B-051), while regions 2 and 3 are for $\sim 70$ hours and $\sim 30$ hours respectively (GBT13A-312). In region 1, we reach a $3 \sigma$ sensitivity of $\mathrm{N}_{\mathrm{HI}} \sim 10^{17.2} \mathrm{~cm}^{-2}$. We reach sensitivities of $\sim 10^{17.6} \mathrm{~cm}^{-2}$ and $\sim 10^{17.8} \mathrm{~cm}^{-2}$ in regions 2 and 3 respectively. The red lines are currently detected $21 \mathrm{~cm}$ sources. 


\section{Chapter 3}

\section{Discrete Clouds of Neutral Gas between the Galaxies M31 and M33 ${ }^{1}$}

\subsection{Introduction}

Spiral galaxies must acquire gas to maintain their observed level of star formation beyond the next few billion years (Leroy et al., 2008). A source of this material may be the gas that resides between galaxies, but our understanding of the state and distribution of this gas is incomplete (Shull et al., 2012). Radio observations (Braun \& Thilker, 2004) of the Local Group of galaxies have revealed hydrogen gas extending from the disk of the galaxy M31 at least halfway to M33. This feature has been interpreted to be the neutral component of a condensing intergalactic filament (Davé et al., 2001) which would be able to fuel star formation in M31 and M33, but simulations suggest that such a feature could also result from an interaction between both galaxies within the past few billion years (Bekki, 2008). Here we report radio observations showing that about 50 percent of this gas is composed of clouds, while the rest is distributed in an extended, diffuse component. The clouds have velocities comparable to those of M31 and M33, and have properties suggesting they are unrelated to other Local Group objects. We conclude that the clouds are likely to be transient condensations of gas embedded in an intergalactic filament and are therefore a potential source of fuel for future star formation in M31 and M33.

\footnotetext{
${ }^{1}$ This chapter has been reproduced from Wolfe et al. (2013).
} 
A recent study (Lockman et al., 2012) of the region between M31 and M33 using the Robert C. Byrd Green Bank Telescope (GBT) at 9' angular resolution has confirmed the presence of neutral hydrogen at levels of a few times $10^{17} \mathrm{~cm}^{-2}$. The study also showed that this neutral gas has a velocity similar to the systemic velocities of M31 and M33, and is thus not the product of confusion with foreground emission from the Milky Way. The sensitivities of the observations were, however, insufficient to provide a detailed understanding of the structure and extent of this gas.

Observations were needed with high angular resolution, high velocity resolution and high sensitivity to distinguish clouds from diffuse gas, and an intergalactic filament from tidal debris. Determining the origin of the gas is important, for if it is from an intergalactic filament, it would represent a new source of material to fuel future star formation in M31 and M33.

\subsection{Preliminary Results}

We have mapped a portion of the hydrogen gas feature between M31 and M33 with improved sensitivity and higher angular and velocity resolution using the GBT. Here we present channel maps of our GBT data (Figure 3.1). The $9^{\prime}$ spectral line data were gridded into a data-cube with the two spatial axes being right ascension and declination, while the third axis is the Local Standard of Rest (LSR) velocity in kilometers-per-second (seen in the upper left of each panel). The rms noise in the map $(1 \sigma)$ is $\sim 4 \mathrm{mK}$ for a $5 \mathrm{~km} \mathrm{~s}^{-1}$ channel. The dashed contour and first solid contour are for $\pm 2 \sigma$ respectively. The other solid contours are for $4 \sigma, 6 \sigma, 8 \sigma$ and $10 \sigma$. There 
is some low level striping evident in the cube. This is primarily due to certain areas of the map having a lower number of scans than others. This effect is only significant at the $2 \sigma$ level. A cloud is visible from -197 to $-223 \mathrm{~km} \mathrm{~s}^{-1}$. Two clouds become visible from -212 to $-254 \mathrm{~km} \mathrm{~s}^{-1}$. Striping in the noise at $-207 \mathrm{~km} \mathrm{~s}^{-1}$ makes it difficult to determine if there is emission there as well. One of these clouds is obviously extended in the northeast-southwest direction. A cloud is visible from -254 to $-310 \mathrm{~km} \mathrm{~s}^{-1}$ and shows a possible extension to the southeast. A cloud is seen from -269 to $-295 \mathrm{~km} \mathrm{~s}^{-1}$. Another is visible from $-285 \mathrm{~km} \mathrm{~s}^{-1}$ to $-310 \mathrm{~km} \mathrm{~s}^{-1}$. A faint cloud can be seen most clearly at -310 and $-316 \mathrm{~km} \mathrm{~s}^{-1}$, but only slightly above $2 \sigma$. This feature is unlikely due to striping since the emission persists over 5 channels, but the striping features only persist (locally) over one or two channels.

We detect seven clouds (Figure 3.2); Table 3.1 lists their properties. The positions and velocities of the brightest clouds are consistent with previous GBT results (Lockman et al., 2012). The primary difference between the results from the Westerbork Synthesis Radio Telescope (WSRT) survey (Braun \& Thilker, 2004), which used that array as a collection of single dishes, and our high resolution data is that here the $\mathrm{H}$ I emission is resolved into features approximately the size of a GBT beamwidth or smaller, and at much higher column densities. The GBT data convolved to $49^{\prime}$ angular resolution reproduce the same basic morphology as the WSRT data. The total $\mathrm{H}_{\mathrm{I}}$ mass detected is $(1.2 \pm 0.1) \times 10^{6} \mathrm{M}_{\odot}$ above a level of $3.0 \times 10^{17} \mathrm{~cm}^{-2},(1.3 \pm$ $0.1) \times 10^{6} \mathrm{M}_{\odot}$ above $1.0 \times 10^{17} \mathrm{~cm}^{-2}$ and $(1.9 \pm 0.1) \times 10^{6} \mathrm{M}_{\odot}$ above $1.0 \times 10^{16} \mathrm{~cm}^{-2}$ (here $\mathrm{M}_{\odot}$ is the solar mass). These values do not appear to be consistent with the relationship between the mass of neutral atomic hydrogen $\left(\mathrm{M}_{\mathrm{HI}}\right)$ and its column 


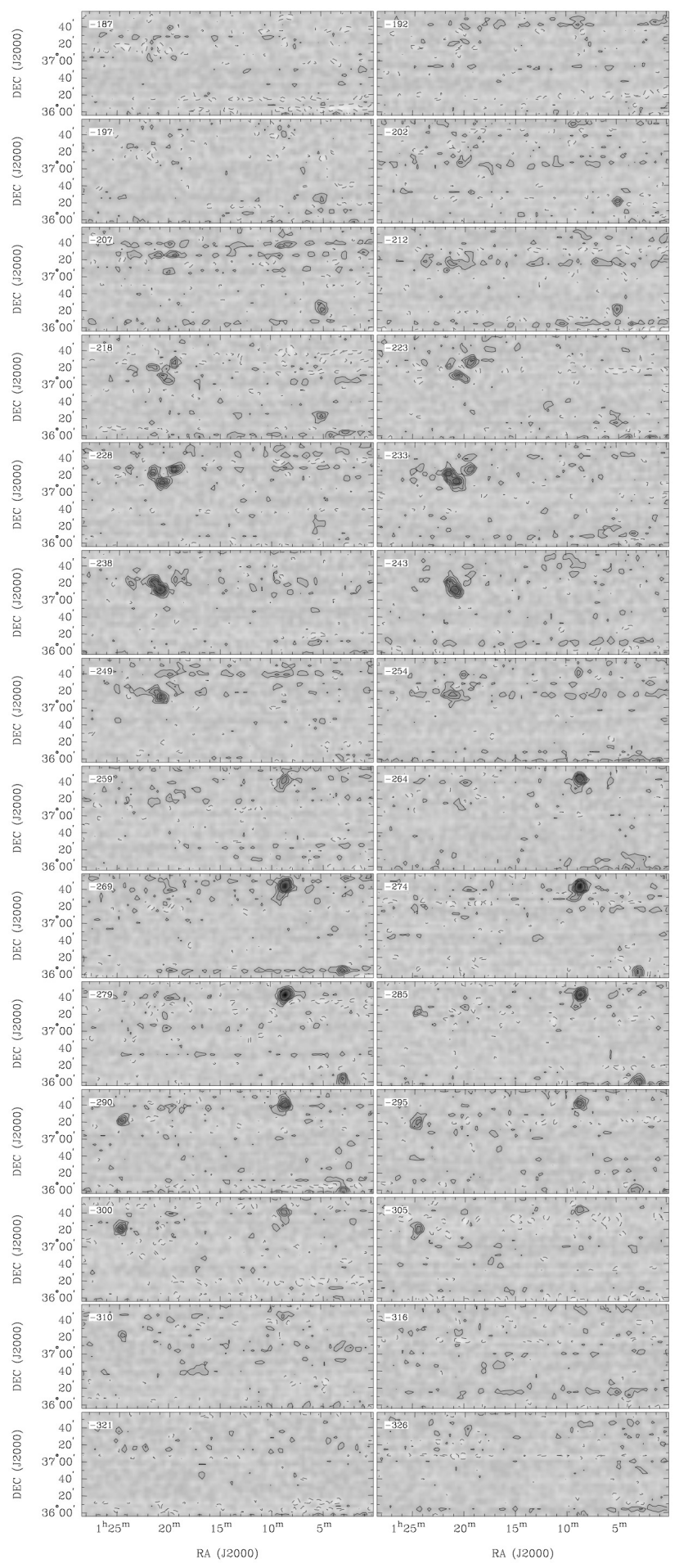

Figure 3.1: Channel maps of the GBT data. The dashed contour and first solid contour are for $\pm 2 \sigma$ $( \pm 8 \mathrm{mK})$ respectively. The other solid contours are for $4 \sigma, 6 \sigma, 8 \sigma$ and $10 \sigma$. 

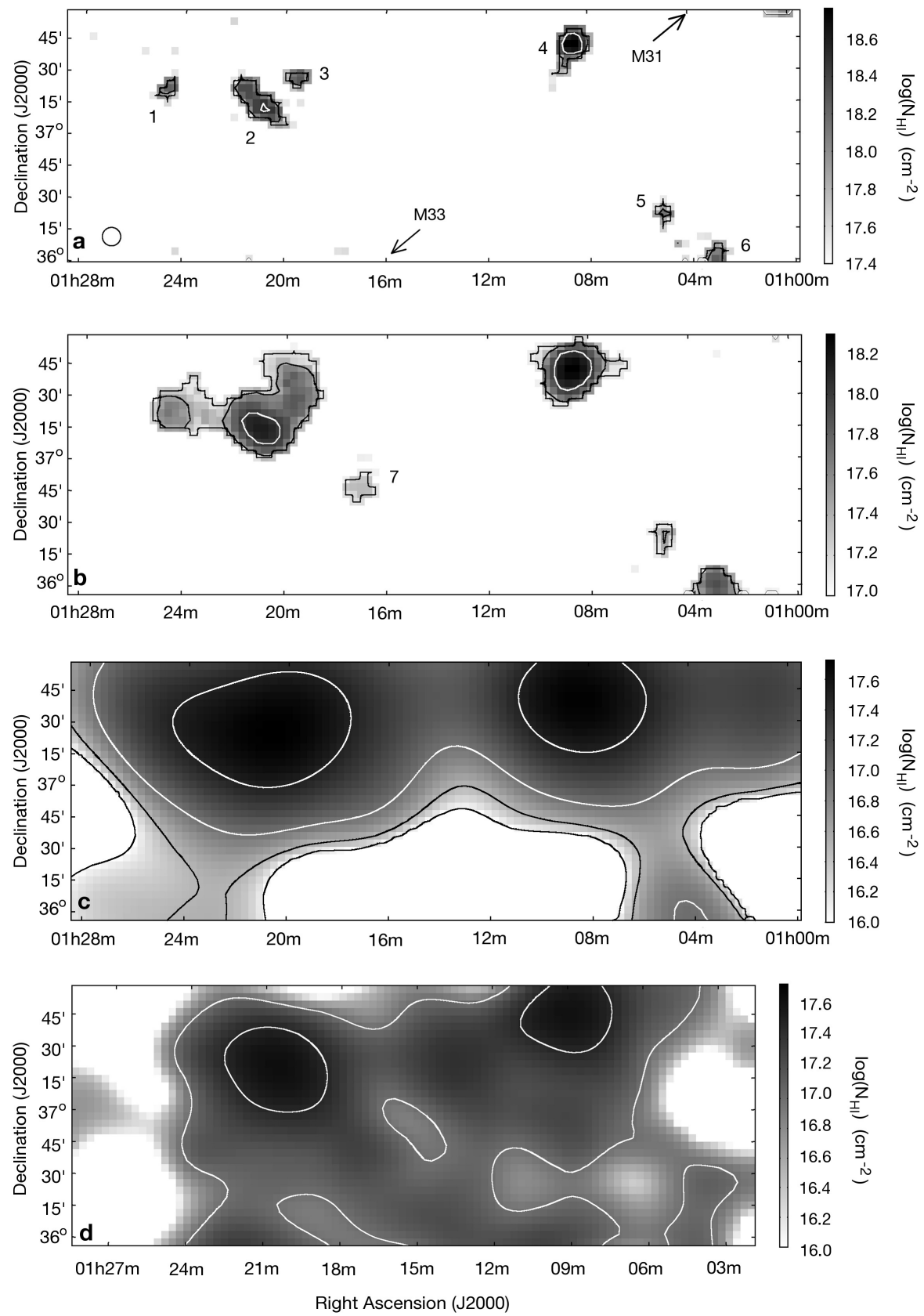

Figure 3.2: Column density maps, in units of $\mathrm{cm}^{-2}$, of $21 \mathrm{~cm}$ emission over a 12 square-degree region between M31 and M33. a: From nearly 250 hours of integration. There are six confirmed $\mathrm{H}$ I clouds, labeled in order of decreasing right ascension. M31 is to the northwest and M33 to the southeast, as indicated by the arrows. b: Smoothed to a $15^{\prime}$ resolution to match the sensitivity of the WSRT data $\left(1 \times 10^{17} \mathrm{~cm}^{-2}\right)$. A seventh cloud is now visible, labeled 7 . The contours are for $\log \left(\mathrm{N}_{\mathrm{HI}}\right)=17.0,17.5,18.0$. c: Smoothed to a $49^{\prime}$ resolution to match the angular resolution of the WSRT data. The contours are for $\log \left(\mathrm{N}_{\mathrm{HI}}\right)=16.0,16.5,17.0,17.5$. d: Part of the WSRT H I survey (Braun \& Thilker, 2004) that first detected neutral gas between M31 and M33, at an angular resolution of $49^{\prime}$ and a velocity resolution of $18 \mathrm{~km} \mathrm{~s}^{-1}$. The contours are for $\log \left(\mathrm{N}_{\mathrm{HI}}\right)=17.0,17.5$. 


\begin{tabular}{|c|c|c|c|c|c|c|c|c|}
\hline $\begin{array}{c}\text { Cloud } \\
\ldots\end{array}$ & \begin{tabular}{|l} 
R.A. \\
H:M:S
\end{tabular} & $\begin{array}{c}\text { DEC } \\
\text { D:M:S }\end{array}$ & $\begin{array}{c}\text { LSR Velocity } \\
\mathrm{km} \mathrm{s}^{-1}\end{array}$ & $\begin{array}{r}\mathrm{FWHM} \\
\mathrm{km} \mathrm{s}^{-1}\end{array}$ & $\begin{array}{c}\mathrm{T}_{\mathrm{B}, \text { Peak }} \\
\mathrm{mK}\end{array}$ & $\begin{array}{c}\log \left(\mathrm{N}_{\mathrm{HI}}\right)_{\text {Peak }} \\
\mathrm{cm}^{-2}\end{array}$ & $\begin{array}{l}\mathrm{M}_{\mathrm{HI}} \\
\mathrm{M}_{\odot}\end{array}$ & $\begin{array}{l}\text { Diameter } \\
( \pm 1) \mathrm{kpc}\end{array}$ \\
\hline 1 & 01:24:42 & $+37: 23: 02$ & $-297 \pm 1$ & $23 \pm 3$ & $47 \pm 4$ & $17.6 \pm 0.04$ & $(1.2 \pm 0.2) \times 10^{5}$ & $\leq 2.8$ \\
\hline 2 & 01:20:52 & $+37: 16: 58$ & $-236 \pm 1$ & $28 \pm 2$ & $69 \pm 4$ & $17.8 \pm 0.03$ & $(3.5 \pm 0.3) \times 10^{5}$ & 6.4 \\
\hline 3 & 01:19:25 & $+37: 31: 12$ & $-226 \pm 2$ & $28 \pm 4$ & $41 \pm 4$ & $17.6 \pm 0.04$ & $(1.1 \pm 0.2) \times 10^{5}$ & $\leq 2.4$ \\
\hline 4 & 01:08:30 & $+37: 44: 51$ & $-277 \pm 1$ & $34 \pm 3$ & $91 \pm 4$ & $17.9 \pm 0.02$ & $(4.0 \pm 0.3) \times 10^{5}$ & 4.8 \\
\hline 5 & 01:05:09 & $+36: 23: 28$ & $-209 \pm 4$ & $26 \pm 3$ & $34 \pm 4$ & $17.5 \pm 0.05$ & $(4.2 \pm 1.0) \times 10^{4}$ & $\leq 2.4$ \\
\hline 6 & 01:03:10 & $+36: 01: 46$ & $-282 \pm 4$ & $29 \pm 3$ & $39 \pm 4$ & $17.6 \pm 0.05$ & $(1.4 \pm 0.2) \times 10^{5}$ & $\leq 3.2$ \\
\hline 7 & 01:17:02 & $+36: 49: 34$ & $-309 \pm 2$ & $24 \pm 4$ & $10 \pm 2$ & $17.0 \pm 0.1$ & $(4.0 \pm 1.0) \times 10^{4}$ & $\leq 3.4$ \\
\hline
\end{tabular}

Table 3.1: A listing of the H I cloud properties. R.A. and DEC are respectively the right ascension and declination of each cloud; LSR, Local Standard of Rest; FWHM, full-width at half-maximum of the $\mathrm{H}$ I line; $\mathrm{T}_{\mathrm{B} \text {,Peak }}$, peak brightness temperature; $\mathrm{N}_{\mathrm{HI} \text {,Peak }}=1.82 \times 10^{18} \mathrm{~T}_{\mathrm{B} \text {,Peak }} \Delta \mathrm{v}$ is the peak column density, where $\Delta \mathrm{v} \approx 5 \mathrm{~km} \mathrm{~s}^{-1} ; \mathrm{M}_{\mathrm{HI}}$ is the $\mathrm{HI}$ mass of the cloud, in solar masses, measured to the $3 \sigma$ column density contour in the maps; diameter is measured to the same contour along the major axis of the cloud, if one is apparent. The $\pm 1 \mathrm{kpc}$ error in the diameter is due to the pixel size of our maps projected to a distance of $\sim 800 \mathrm{kpc}$. The values for cloud 7 were obtained from the $15^{\prime}$ map. Quantities were calculated assuming a distance to the clouds of $800 \mathrm{kpc}$ (Lockman et al., 2012).

density $\left(\mathrm{N}_{\mathrm{HI}}\right)$ predicted by simulations of intergalactic $\mathrm{H}$ I at low redshift (Popping et al., 2009). The total H mass measured across the entire map is $(2.6 \pm 0.2) \times 10^{6}$ $\mathrm{M}_{\odot}$, identical to the total H i mass from the WSRT data over the same area. There is probably additional mass present in the form of ionized gas, as at such low column densities photoionization of the $\mathrm{H}_{\mathrm{I}}$ from the extragalactic radiation field becomes significant (Maloney, 1993).

About $50 \%$ of the neutral hydrogen is in clouds, whose diameters range from $\leq 2.4 \mathrm{kpc}$ to $6.4 \mathrm{kpc}$. This is the size range of dwarf galaxies; however, although stellar surveys (Ibata et al., 2007; McConnachie et al., 2009) have detected dwarf galaxies around M31 as well as potential dwarf galaxy remnants, there are no reports of stellar over-densities at the positions of these clouds (McConnachie et al., 2009), though there is no lack of stellar streams in the general vicinity (Lewis et al., 2013). The clouds lie much farther from M31 and M33 than each galaxy's respective high velocity cloud (HVC) population and also lie closer to the systemic velocities of M31 


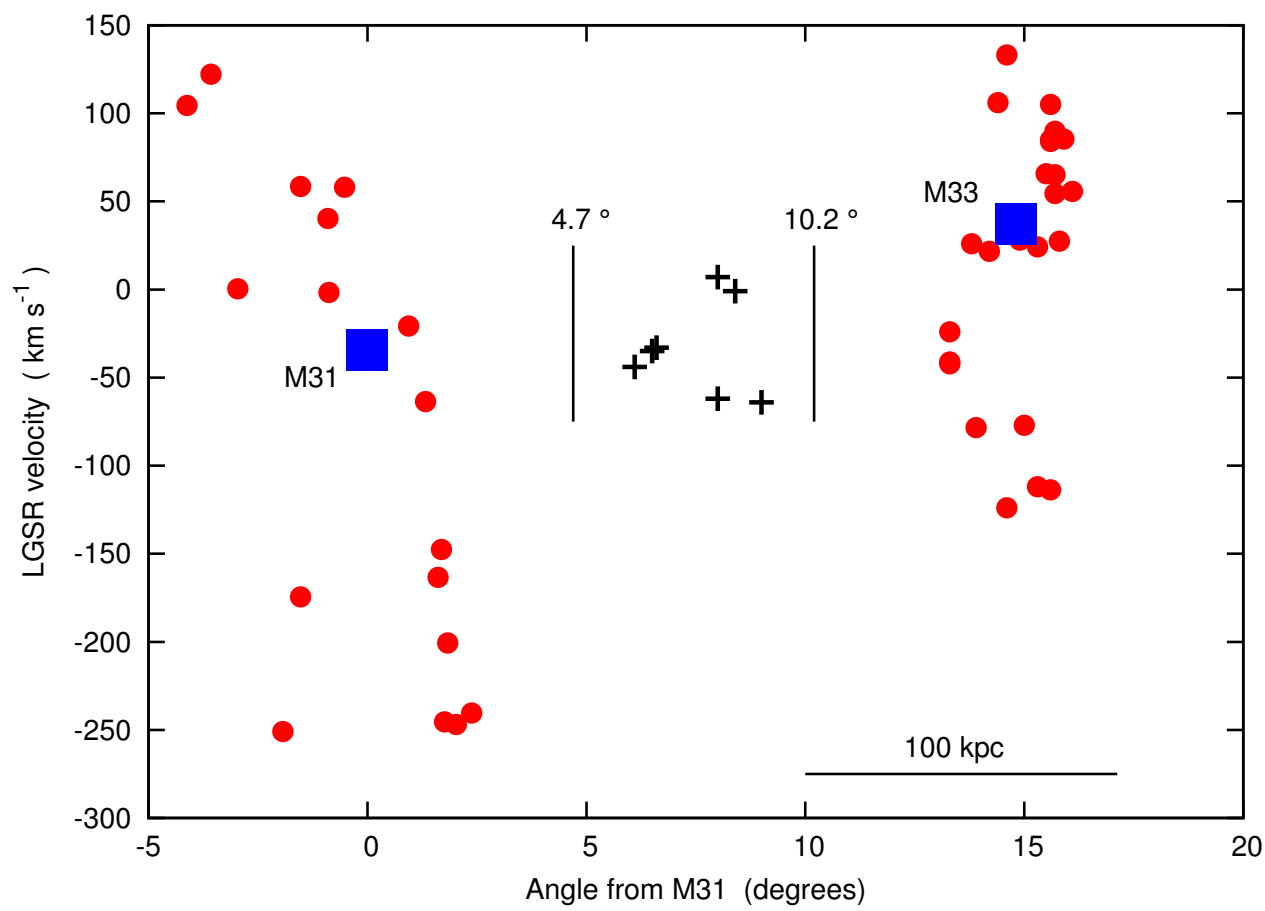

Figure 3.3: A position-velocity plot of the H I clouds and selected Local Group objects. The local group standard of rest (LGSR) velocities were calculated using the NASA Extragalactic Database. Large blue squares indicate M31 and M33, both labeled. Filled red circles are the high velocity clouds of each galaxy Thilker et al. (2004); Grossi et al. (2008); Westmeier et al. (2008); Putman et al. (2009). Crosses show clouds detected in the GBT maps; the vertical bars mark the GBT map boundaries, in terms of angle from M31. The HVCs lie within $\sim 50 \mathrm{kpc}$ of each galaxy, whereas new clouds are found $\gtrsim 100 \mathrm{kpc}$ from both galaxies. The clouds also have an inter-cloud velocity dispersion of $25 \mathrm{~km} \mathrm{~s}^{-1}$, compared to the inter-cloud velocity dispersion of $70-130 \mathrm{~km} \mathrm{~s}^{-1}$ for the HVCs.

and M33 than the HVCs (Figure 3.3). The clouds are also kinematically cooler as a population than the HVCs, with a lower inter-cloud velocity dispersion by a factor of two to five.

We estimate the baryonic mass and circular rotation speed about the cloud center for the clouds as well as for the HVCs and dwarf galaxies of M31 and M33, and plot them with the baryonic Tully-Fisher relation (Tully \& Fisher, 1977; McGaugh et al., 2000) (Figure 3.4). This allows us to compare the clouds to other members of the Local Group. The clouds are offset from the Tully-Fisher relation for rotating 
disk galaxies; they occupy a similar region in the Tully-Fisher plane as the HVCs and dwarfs, but cluster more tightly.

\subsection{Virial Mass and Survival Times}

If the linewidths of the clouds are interpreted as an equilibrium velocity representative of the gravitational potential, then the clouds would be highly dark matter dominated. The virial mass of every cloud is two to three orders of magnitude larger than its H I mass. The presence of an ionized component, however, would decrease the amount of dark matter necessary to match the virial mass. The free-fall time for these clouds, or time it would take for these clouds to freely collapse under their own gravity, is $\sim 400$ Myr and the crossing time, the time it would take for a parcel of gas to cross the length of the cloud, is $\sim 100$ Myr. These times are quite short to have resulted from the interaction between M31 and M33 proposed to have occurred a few billion years ago (Bekki, 2008; McConnachie et al., 2009) unless confined by an external pressure. The warm-hot intergalactic medium (WHIM) (Davé et al., 2001) at a temperature $\sim 10^{6} \mathrm{~K}$ and density $\gtrsim 10^{-7} \mathrm{~cm}^{-3}$ would supply the pressure if the clouds have a temperature of $\sim 10^{4} \mathrm{~K}$, consistent with the values inferred from the cloud linewidths. The WHIM densities needed are comparable to those predicted (Fukugita \& Peebles, 2004) beyond the virialized region of galaxies. Therefore, these clouds could be in complete pressure equilibrium with the WHIM. 


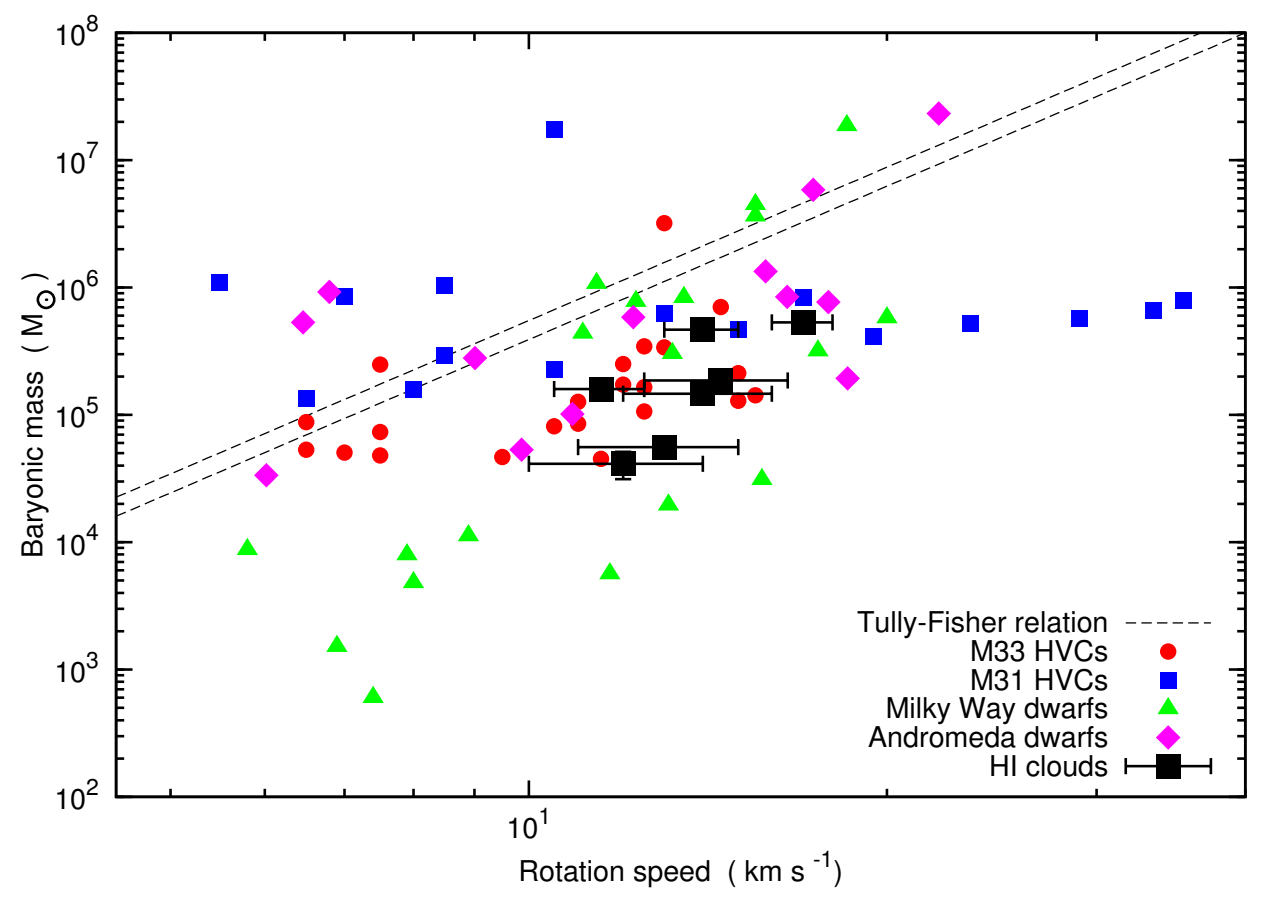

Figure 3.4: A plot of baryonic mass versus equivalent circular rotation speed. This figure compares the newly detected clouds (black filled squares with $1 \sigma$ errorbars), the HVCs of M31 and M33 (squares, circles), Milky Way dwarf satellite galaxies (McGaugh \& Wolf, 2010) (triangles) and the Andromeda dwarfs (Tollerud et al., 2012) (diamonds) to the baryonic Tully-Fisher relation (McGaugh, 2012) with $\pm 1 \sigma$ uncertainty (dashed lines). We assume that all of the mass is in the neutral atomic gas and that $\mathrm{M}_{\text {baryonic }}=1.33 \times \mathrm{M}_{\mathrm{HI}}$ to account for helium. The masses are plotted in units of solar masses. The presence of an ionized component will increase the baryonic mass and move the clouds closer to the relation, but we have no way at present to estimate its magnitude. The effective circular rotation speed is $\mathrm{v}_{\text {rot }}=\mathrm{FWHM} / 2$ where FWHM is the full width at half-maximum of the $\mathrm{HI}$ line. This assumption holds in the case where the gas may be in rotation or is a turbulently supported, spherical system with an assumed harmonic radius of $\mathrm{R} / 2$, where $\mathrm{R}$ is the cloud radius, measured to same column density contour as the diameter in Table 1. The scatter in mass-rotation velocity is 0.5 dex for the clouds, 0.8 dex for the Andromeda dwarfs and M33's HVCs, 1.2 dex for M31's HVCs, and 1.8 dex for the Milky Way dwarfs. The average errors (in dex) for the rotation velocities $\left\langle\sigma_{v}\right\rangle$ and the masses $\left\langle\sigma_{M}\right\rangle$ are respectively 0.05 dex and 0.1 for M33's HVCs, 0.05 and 0.06 for M31's HVCs, 0.1 and 0.2 for the Milky Way dwarfs, 0.1 and 0.7 for the Andromeda dwarfs and 0.06 and 0.08 for the H I clouds. 


\subsection{Cloud Origins}

These clouds have several possible origins. First, they may be primordial, gasrich objects similar to the dwarf spheroidal or dwarf irregular galaxies in the Local Group, but this does not explain why the gas seems to lie along a connecting structure, even when resolved into clouds. Second, the clouds may be gas that is accreting onto sub-halos. This might occur naturally if the gas is part of a cosmic filament. If instead the gas is tidal in origin, it is likely to be moving at relative speeds that exceed the escape velocities of sub-halos, making accretion unlikely. Third, the clouds may be analogous to tidal dwarf galaxies (Gentile et al., 2007). This would account for their spatial and kinematical distribution with respect to M31 and M33. If so, the clouds would need to be in pressure equilibrium with the WHIM, so that they could persist for a few billion years following the interaction between M31 and M33. It's also possible that there may have been a more recent interaction that produced these clouds, such as M31 with IC10 or some other object. These clouds contain no evidence of stars, however, and do not fall on the Tully-Fisher relation, as one system of tidal dwarfs is known to do (Gentile et al., 2007). Last, the clouds could be transient objects condensing from an intergalactic filament, as originally proposed (Braun \& Thilker, 2004; Maller \& Bullock, 2004). This could explain their spatial distribution, as well as the lack of stellar overdensities. A recent simulation (Fernández et al., 2012) of a Milky Way sized galaxy has produced analogous material out to $\sim 100 \mathrm{kpc}$ with H I masses $\sim 10^{5} \mathrm{M}_{\odot}$, suggesting that the clouds are most probably condensations from an intergalactic filament. 


\subsection{Conclusions}

We conclude that the H i clouds between M31 and M33 are a unique population, lacking a clear analog in other members of the Local Group, such as the dwarf galaxies or HVCs. Although our current results suggest that the clouds may arise in an intergalactic filament, and thus are potential fuel to feed star formation in M31 and/or M33, the question of their origin needs further consideration. The new clouds we report here were discovered in only about half of our survey time and thus are not at the full sensitivity that we intend to achieve. The region we have studied is only a fraction of the area around M31 reported (Braun \& Thilker, 2004) to have diffuse H I, so the clouds measured here may be the first representatives of a much larger population. 


\section{Chapter 4}

\section{The Diffuse $\mathrm{H}_{\text {I }}$ around M31}

Here we discuss the results of the full 400 hour GBT survey between M31 and M33. We also discuss results from a set of deep, pointed observations to the northwest of M31 to search for similar material as seen in the Braun \& Thilker (2004) maps. We use this new information to try and develop a better understanding of the origins of the diffuse $\mathrm{H}$ I and how it may be related to the other Local Group objects.

\subsection{Observations}

First, to complete our 400 hour survey, the last 150 hours of GBT data were reduced and added to our previous data cube. Since these data were part of the same set of observations for the previous data cube, we refer the reader to Chapter 2 for the discussion of the telescope and backend configuration, as well as the reduction scheme.

In short, we observed at $1.4 \mathrm{GHz}$ using the GBT Spectrometer with a bandwidth of 12.5 $\mathrm{MHz}$ and a velocity resolution of $0.3 \mathrm{~km} \mathrm{~s}^{-1}$ that was later smoothed to $5 \mathrm{~km} \mathrm{~s}^{-1}$.

Second, we were also awarded 100 hours of GBT time (GBT13A-312) to map two 12 square degree regions to the north of our original area (Fig. 2.1). Unfortunately, this was not enough time to map the area to the desired sensitivity. In the upper map, we reach a sensitivity limit in $\mathrm{H}_{\mathrm{I}}$ of $\mathrm{N}_{\mathrm{HI}}=7.0 \times 10^{17} \mathrm{~cm}^{-2}$ and $\mathrm{N}_{\mathrm{HI}}=4.4 \times$

$10^{17} \mathrm{~cm}^{-2}$ in the lower map. These limits assume a $25 \mathrm{~km} \mathrm{~s}^{-1}$ linewidth. No features 
of significance appear in these areas. Observations of the region are ongoing, so we will postpone a discussion until better sensitivities can be reached.

Lastly, we were awarded 50 hours of GBT time to conduct deep pointings to the northwest of M31. While some telescope time was devoted to finishing the mapping of the region between M31 and M33, we nevertheless conducted 19 pointings as indicated in Figure 4.1. The observations were done at $1.4 \mathrm{GHz}$ and frequency switched with a bandwidth of $12.5 \mathrm{MHz}$ using a frequency throw of $4 \mathrm{MHz}$. The average total integration time for each pointing is 76 minutes. The data reduction was carried out with the standard GBTIDL routines. After the calibration of the data, a second order baseline was fit over the spectrum to remove any large scale baseline structure. The fit was done over velocities believed not to contain any emission. The data were then boxcar smoothed from $0.3 \mathrm{~km} \mathrm{~s}^{-1}$ resolution to $1.3 \mathrm{~km} \mathrm{~s}^{-1}$ to further increase sensitivity. Each pointing was 10 minutes in length and multiple pointings were averaged to produce the final spectra.

\subsection{Results}

\subsubsection{The 400 Hour Survey}

Upon inclusion of the final 150 hours of data from our survey, the 12 square degree region between M31 and M33 reached a spectral noise of approximately 2.5 mK over a $5 \mathrm{~km} \mathrm{~s}^{-1}$ channel, which corresponds to a $3 \sigma$ column density sensitivity in $\mathrm{H}$ I of $\mathrm{N}_{\mathrm{HI}}=1.6 \times 10^{17} \mathrm{~cm}^{-2}$ over a $25 \mathrm{~km} \mathrm{~s}^{-1}$ linewidth. An integrated intensity 


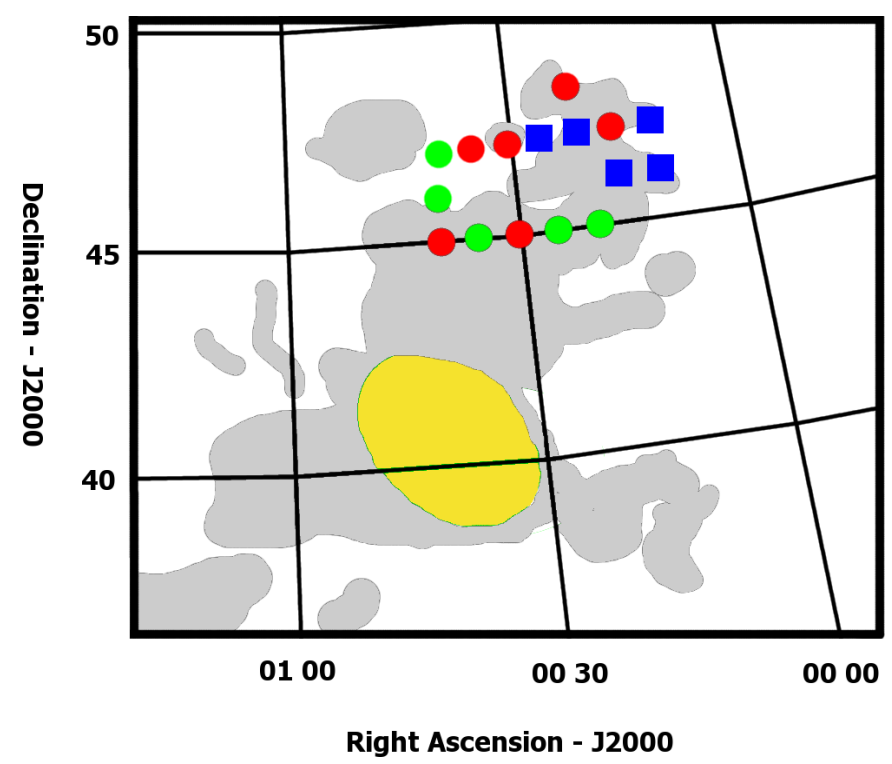

Figure 4.1: A simple figure showing the approximate locations of our GBT pointings from GBT12A266. Yellow is the primary emission from M31, while the grey is the extended emission as seen in the Braun \& Thilker (2004) map. Blue squares indicate detections of faint H I emission. Red circles are non detections and the green circles are Milky Way emission with velocities similar to those of the detections.

map is presented in Figure 4.2. We label the clouds here as we do in Chapter 3. A table of the cloud properties is shown in Table 4.1.

As seen in the total integrated intensity map, more diffuse emission appears in the clouds. The total $\mathrm{H}_{\mathrm{I}}$ mass of all seven clouds is $(2.3 \pm 0.05) \times 10^{6} \mathrm{M}_{\odot}$, which is similar to the total H I mass as seen in the Braun \& Thilker (2004) map when measured over the same area and roughly the same sensitivity (Figure 3.1). This means that all the emission seen in the Braun \& Thilker (2004) map is in the form of clouds, as opposed to only $50 \%$ from our earlier results.

Clouds 1 and 7 show extended features. Cloud 4 clearly shows head-tail structure. A small extension is seen to the southwest also, but is at low significance. Clouds 2 and 3 also show a connection at the $3 \sigma$ level. The concentration in the upper right corner is a gridding artifact. 


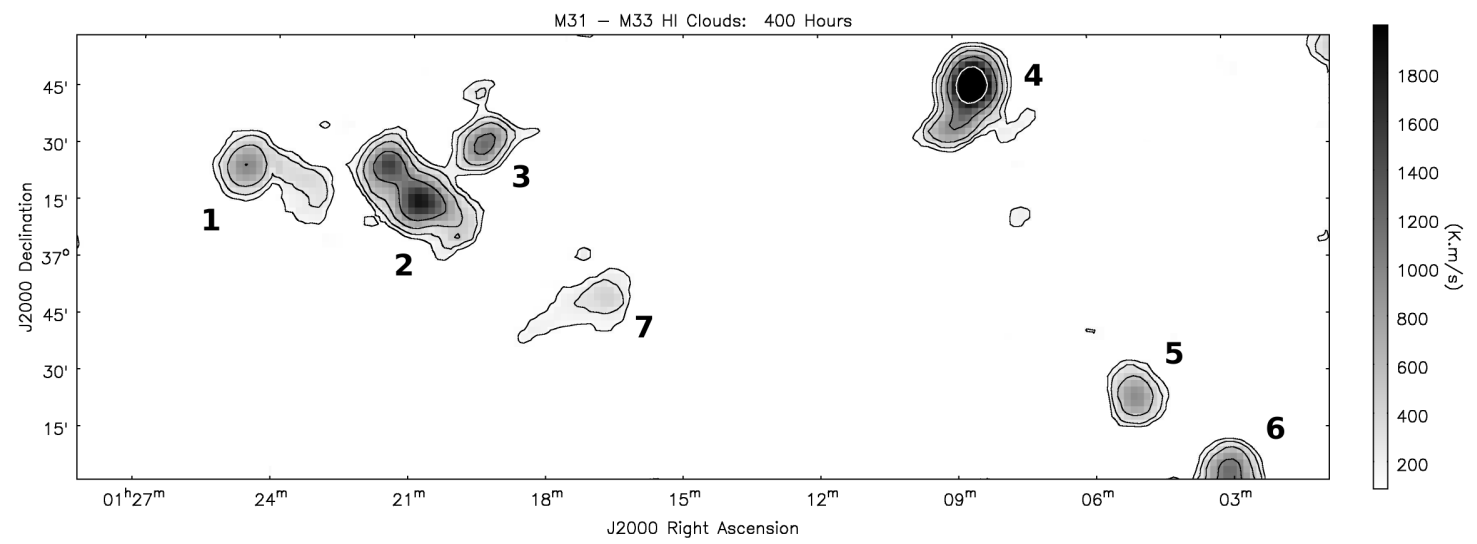

Figure 4.2: Total intensity, or moment-0, map of the M31-M33 H I clouds. The map was created by blanking the cube below the $3 \sigma$ noise limit, then adding up all emission over the relevant velocity range (-320 to $-180 \mathrm{~km} \mathrm{~s}^{-1}$ in the LSRK frame). The contours are for 3, 6, 12 and $24 \sigma$.

\subsubsection{Velocities and Linewidths}

We also present the intensity weighted kinematic local standard of rest (LSRK) velocity fields, or moment-1 maps, of the clouds to search for any structures in the kinematics of the clouds. These maps were made by blanking the data cubes to only include emission above the $3 \sigma$ noise limit. The LSRK velocity accounts for the peculiar motion of the Sun with respect to the rotation of the Galaxy. These maps were created using the MIRIAD task moment. See Figure 4.3 for a velocity field of the whole region. We also present the velocity fields of clouds one through five (Figures 4.4-4.7).

The extension for cloud 1 is offset in velocity from the main core of the cloud by $65 \mathrm{~km} \mathrm{~s}^{-1}$ and is more closely related, kinematically, to clouds 2 and 3 . The extension could be a separate cloud that is spatially coincident with cloud 1 or may be material that has been stripped from cloud 1 , or perhaps even from clouds 2 or 3 . There is a small gradient across this extension, but it is only a few channels wide. Clouds 2 


\begin{tabular}{c|c|c|c|c|c|c|}
$\begin{array}{c}\text { Cloud } \\
\ldots\end{array}$ & $\begin{array}{c}\text { R.A. } \\
\text { H:M:S }\end{array}$ & $\begin{array}{c}\text { DEC } \\
\text { D:M:S }\end{array}$ & $\begin{array}{c}\text { FWHM } \\
\mathrm{km} \mathrm{s}^{-1}\end{array}$ & $\begin{array}{c}\mathrm{N}_{\mathrm{HI}} \\
\times 10^{18} \mathrm{~cm}^{-2}\end{array}$ & $\begin{array}{c}\mathrm{M}_{\mathrm{HI}} \\
\times 10^{5} \mathrm{M}_{\odot}\end{array}$ & $\begin{array}{c}\text { Diameter } \\
\pm 1 \mathrm{kpc}\end{array}$ \\
\hline 1 & $01: 24: 42$ & $+37: 23: 02$ & $24 \pm 3$ & $1.8 \pm 0.2$ & $2.8 \pm 0.2$ & 7.5 \\
2 & $01: 20: 52$ & $+37: 16: 58$ & $28 \pm 3$ & $3.4 \pm 0.1$ & $13.7 \pm 0.2$ & 8.8 \\
3 & $01: 19: 25$ & $+37: 31: 12$ & $28 \pm 3$ & $2.2 \pm 0.2$ & - & 4.3 \\
4 & $01: 08: 30$ & $+37: 44: 51$ & $34 \pm 3$ & $5.5 \pm 0.1$ & $11.6 \pm 0.1$ & 7.7 \\
5 & $01: 05: 09$ & $+36: 23: 28$ & $27 \pm 3$ & $1.7 \pm 0.1$ & $1.8 \pm 0.1$ & 4.4 \\
6 & $01: 03: 10$ & $+36: 01: 46$ & $29 \pm 3$ & $2.2 \pm 0.2$ & $2.6 \pm 0.2$ & 4.0 \\
7 & $01: 17: 02$ & $+36: 49: 34$ & $24 \pm 3$ & $1.2 \pm 0.1$ & $0.97 \pm 0.1$ & 7.4
\end{tabular}

Table 4.1: The H I cloud properties for the entire 400 hour, 12 square degree survey. FWHM is the full width at half maximum of the $\mathrm{H}$ I line measured at the peak brightness temperature in the data cube. R.A. and DEC are the right ascension and declination of the cloud respectively. $\mathrm{N}_{\mathrm{HI}}$ is the column density of the cloud, integrated over the spectral line where the brightness temperature of the cloud peaks. $\mathrm{M}_{\mathrm{HI}}$ is the H I mass of the cloud, in solar masses, measured to the $3 \sigma$ column density contour in the maps. Quantities were calculated assuming a distance to the clouds of 800 kpc (Lockman et al., 2012). The diameter is measured across the major axis of the cloud, if one is apparent, down to the $3 \sigma$ contour. Since clouds 2 and 3 now show a connection, their Hi masses are added under the entry for cloud 2 .

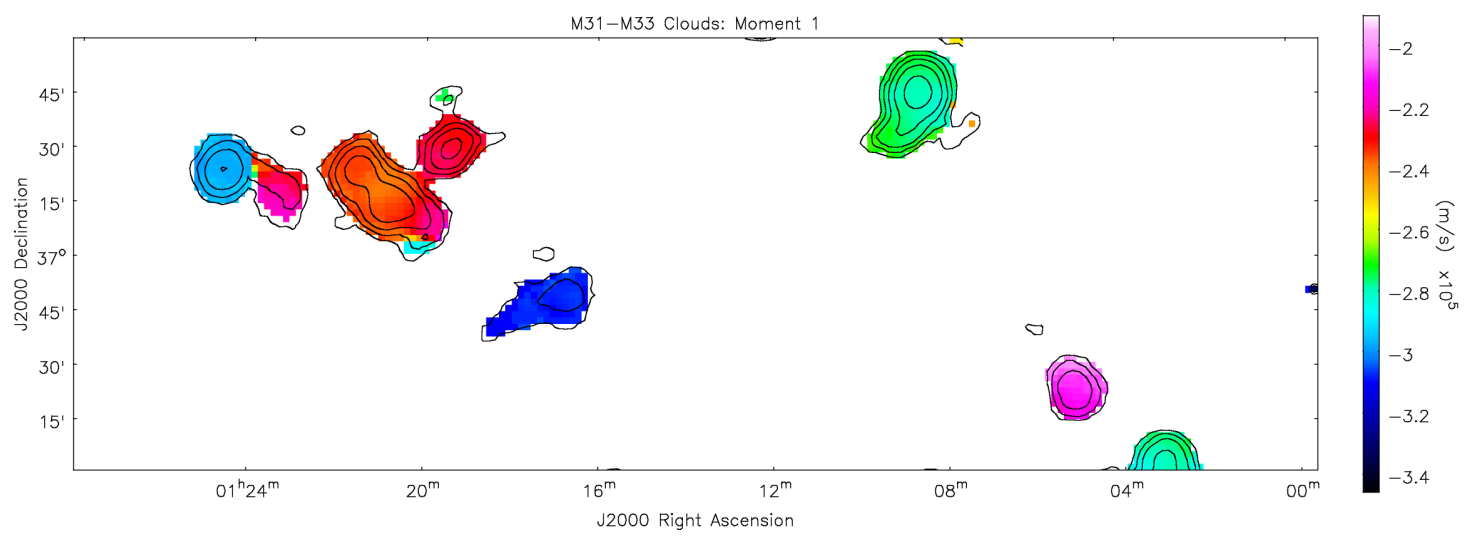

Figure 4.3: Velocity field for the H I clouds. The contours are from the intensity map (Fig. 4.2).

and 3 appear to be kinematically related, at similar velocities, and there are small gradients across clouds 3 and 5 and in the southwest extension of cloud 2. Again, however, these gradients are only a few velocity channels wide. Cloud 4 shows a small gradient across its tail as well, but clouds 6 and 7 show no signs of any structure.

We also searched for line broadening across the clouds. Line broadening is when the linewidth of the emission increases across the cloud, and occurs when the 


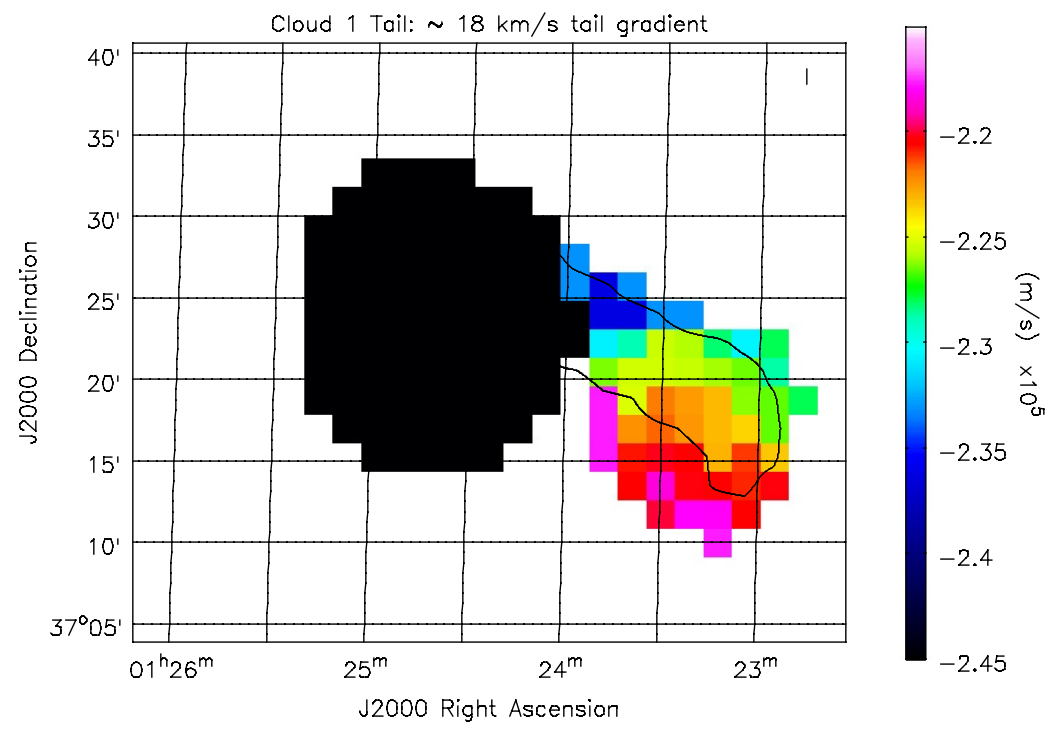

Figure 4.4: Velocity field for cloud 1 . The contour is at $0.3 \mathrm{~K} \mathrm{~km} \mathrm{~s}^{-1}$. The main body of the cloud is offset from the extension in velocity and shows no structure.

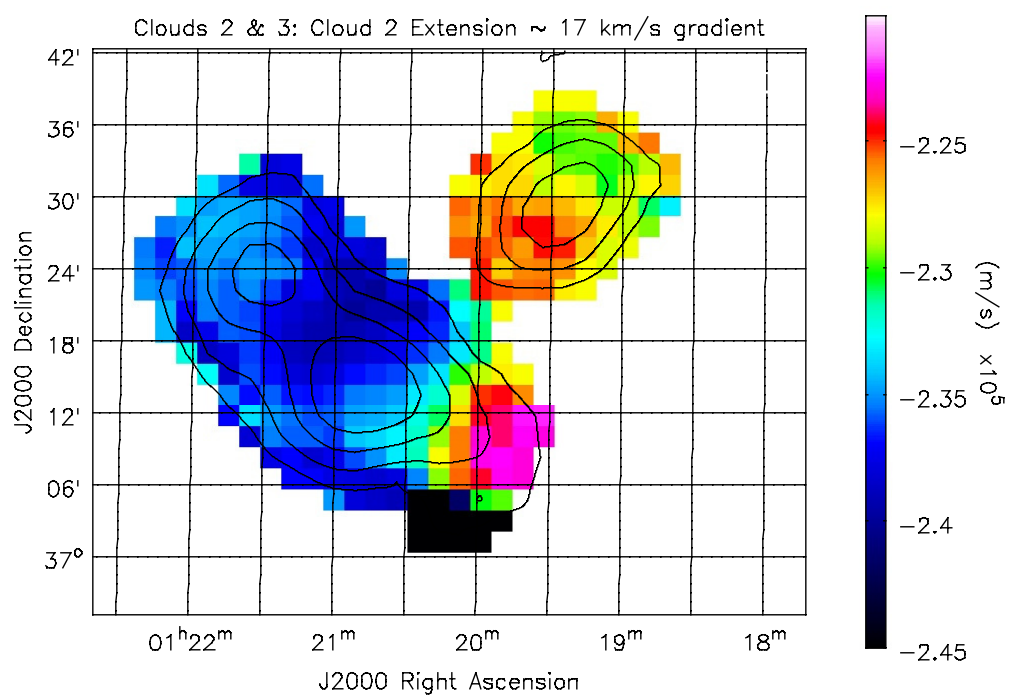

Figure 4.5: Velocity field for clouds $2 \& 3$. The contours are at $0.4,0.7,1.0,1.3,1.8$ and 2.7 $\mathrm{K} \mathrm{km} \mathrm{s}^{-1}$.

gas is heated. This could be due to some type of interaction between the cloud and surrounding environment. If so, one would expect an increase in the line width of the spectra as one moves away from the center of the cloud and toward the tail, or 


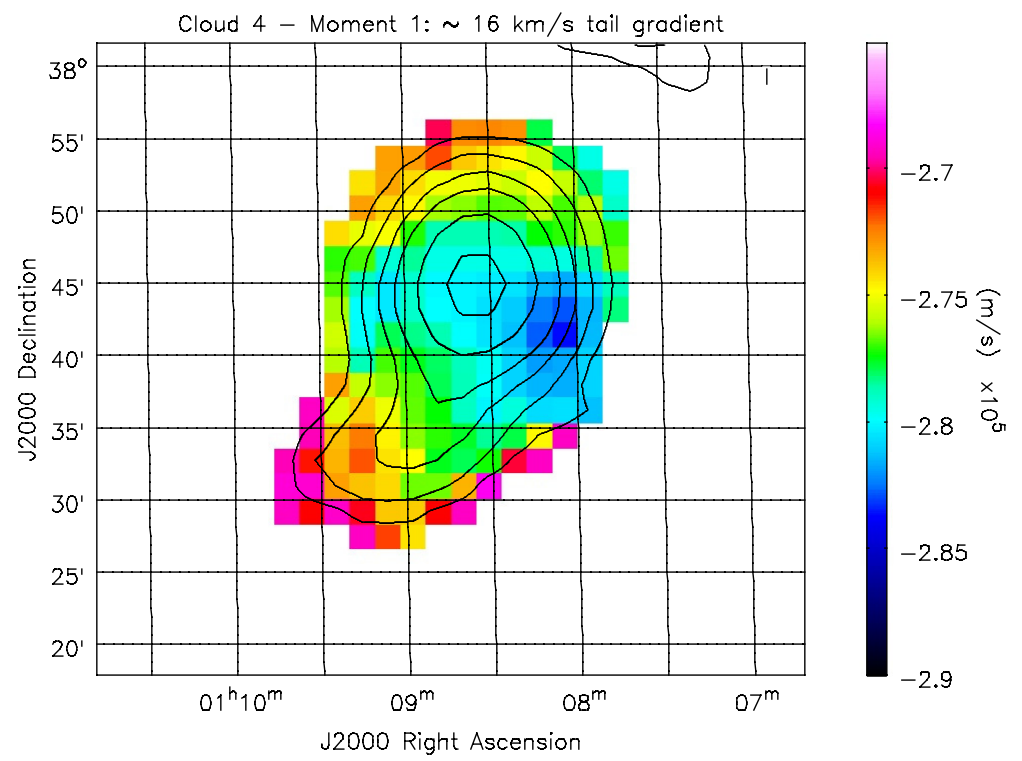

Figure 4.6: Velocity field for cloud 4. The contours are at $0.4,0.7,1.0,1.3,1.8$ and $2.7 \mathrm{~K} \mathrm{~km} \mathrm{~s}^{-1}$.

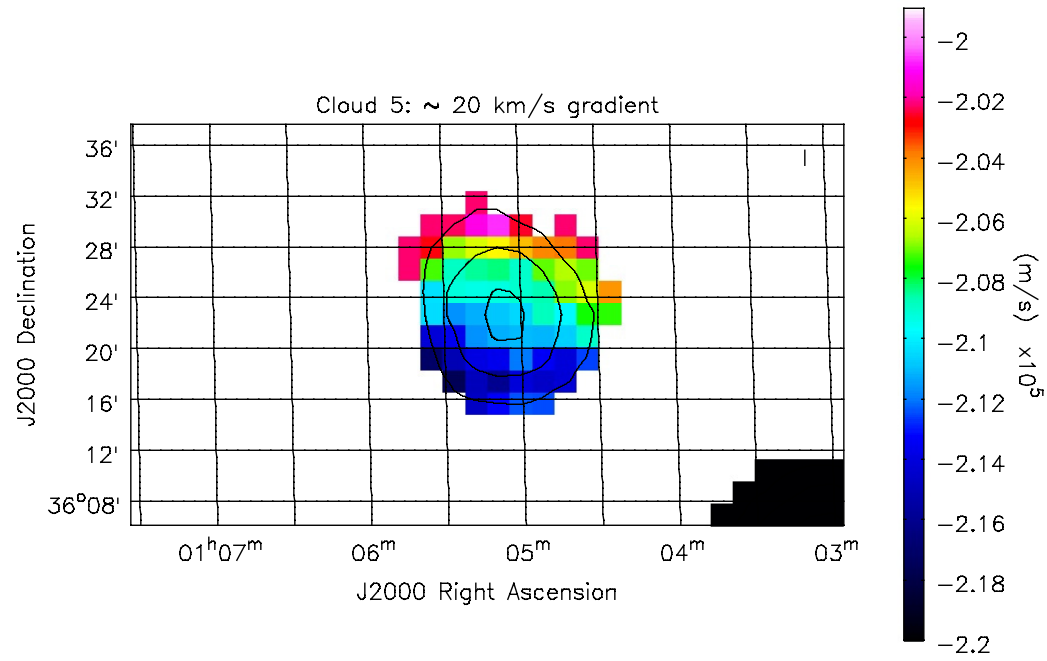

Figure 4.7: Velocity field for cloud 5. The contours are at $0.4,0.7$ and $0.8 \mathrm{~K} \mathrm{~km} \mathrm{~s}^{-1}$.

other extensions (e.g. Brüns \& Mebold, 2004; Westmeier et al., 2005). After a close inspection of the linewidths across each cloud using the AIPS task PLCUB as well as visual inspection of the spectra, no discernible line broadening is present in any of these clouds. Spectra from PLCUB for each cloud are presented in Figures 4.8-4.13. 


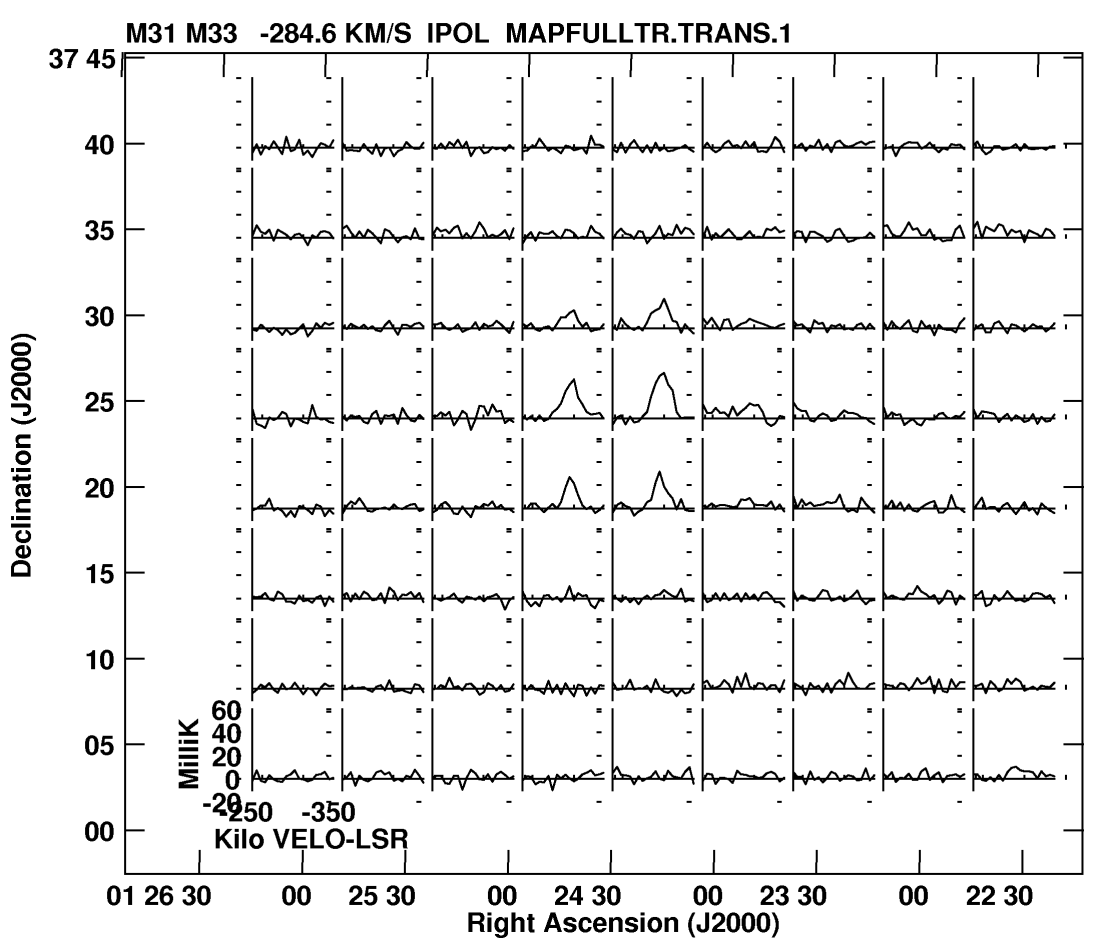

Figure 4.8: H I spectra across cloud 1 using the AIPS task PLCUB. Each spectrum is taken every $5^{\prime}$ in both right ascension and declination.

It may be the case that our velocity resolution, $\sim 5 \mathrm{~km} \mathrm{~s}^{-1}$ is simply to coarse to detect line broadening. Based on this data, there is no conclusive evidence of interaction between the clouds and surrounding medium. Spectra with higher velocity resolution and maps with higher spatial resolution are needed.

\subsubsection{The Pointings to the Northwest of M31}

The result from our pointings to the northwest of M31 are shown in Table 4.2. The spectra from the pointings are shown in Figures 4.14-4.19. Given the amount of time awarded for these observations, we first picked locations further apart and then began to fill in a grid of pointings. Roughly $30 \%$ of the detections are from the Milky

Way, where the Galactic spectra extend to $\mathrm{V}_{\mathrm{LSRK}}$ of $\sim-150 \mathrm{~km} \mathrm{~s}^{-1}$ in the form of 


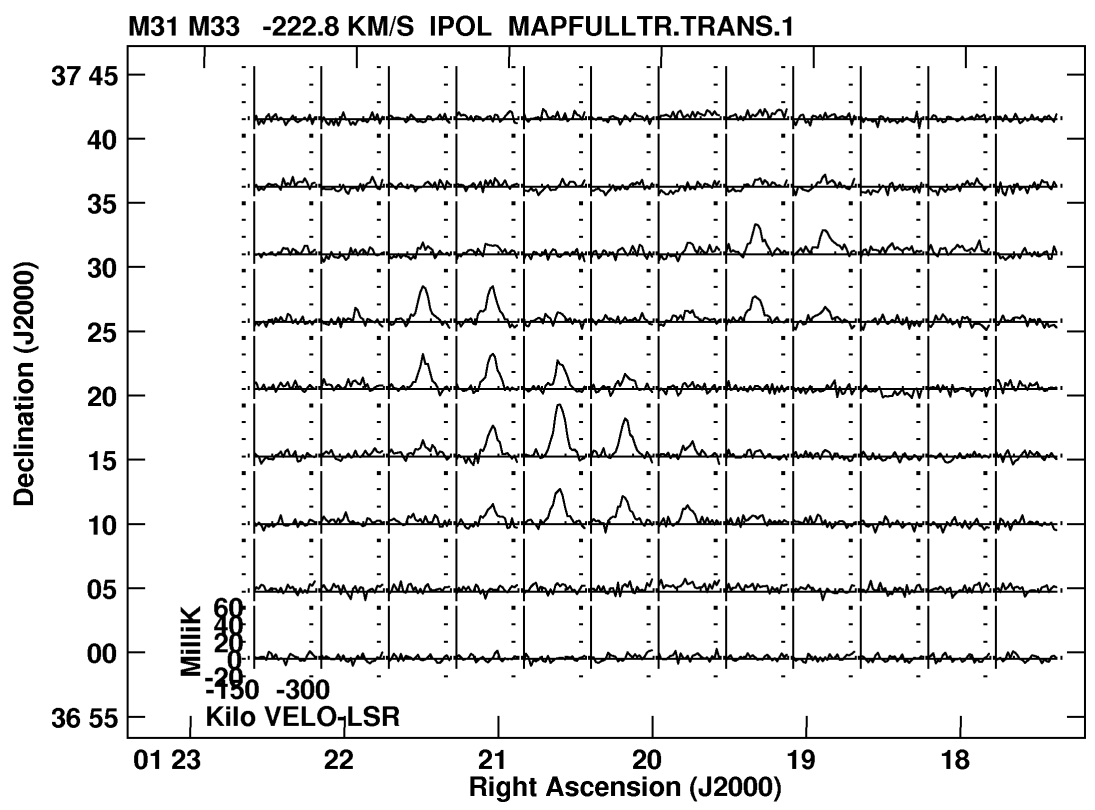

Figure 4.9: H I spectra across clouds 2 and 3 using the AIPS task PLCUB. Each spectrum is taken every $5^{\prime}$ in both right ascension and declination.

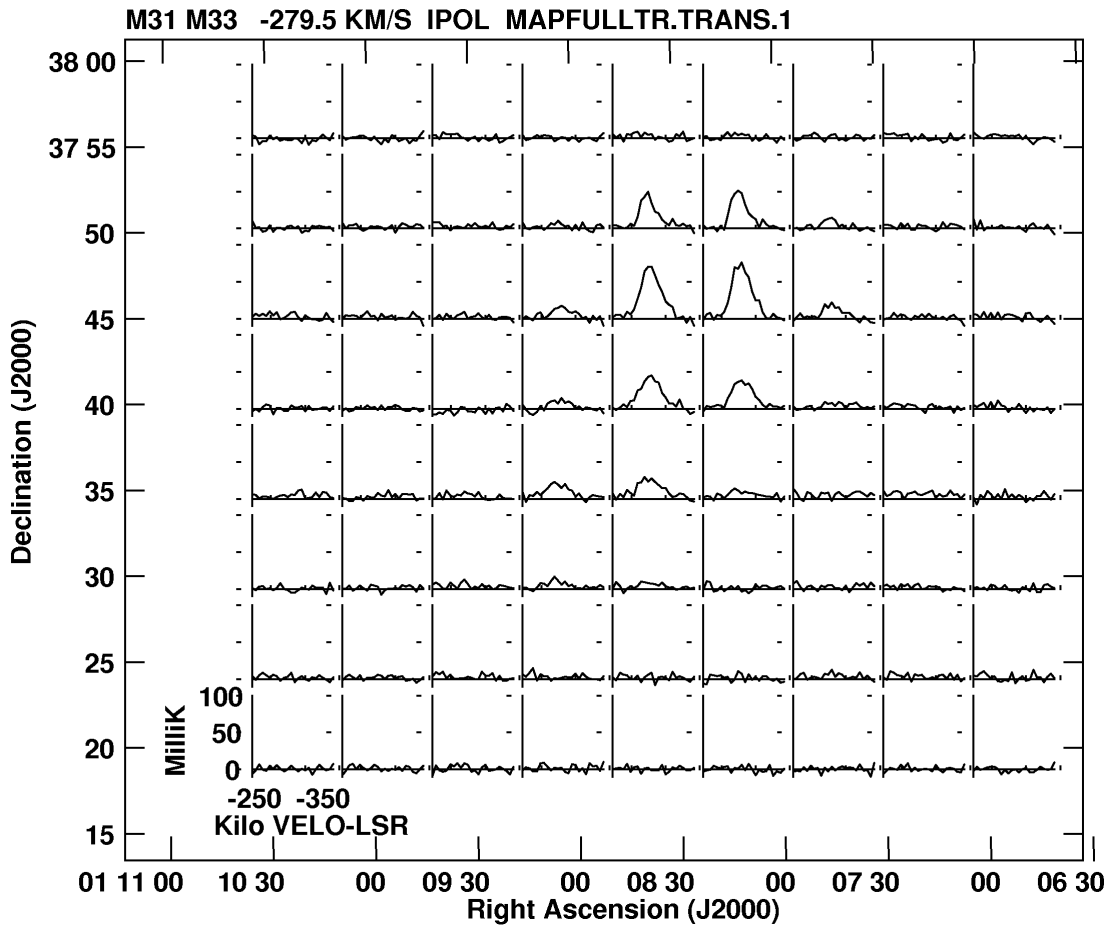

Figure 4.10: H I spectra across cloud 4 using the AIPS task PLCUB. Each spectrum is taken every $5^{\prime}$ in both right ascension and declination. Each spectrum is taken every $5^{\prime}$ in both right ascension and declination. 


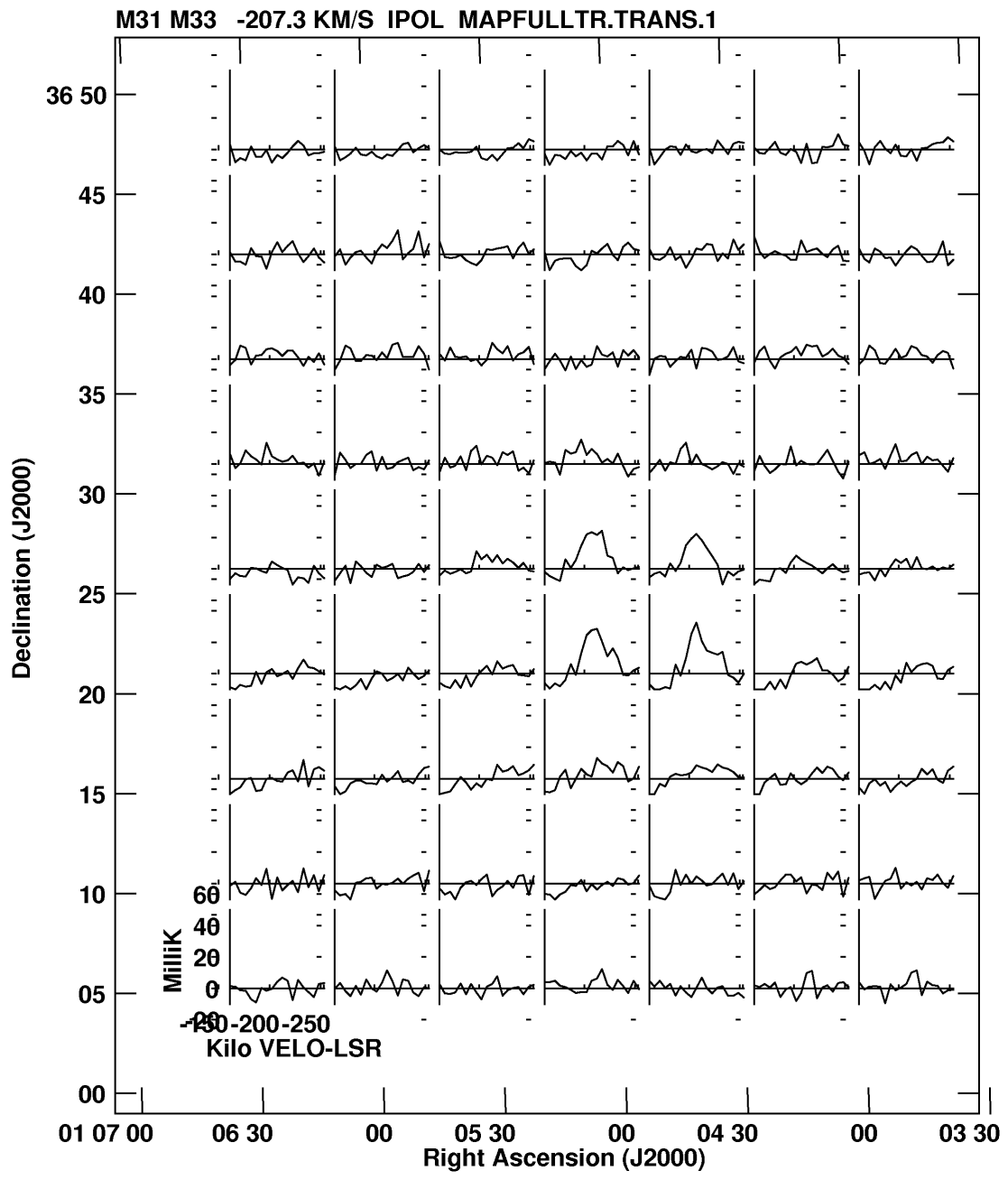

Figure 4.11: H I spectra across cloud 5 using the AIPS task PLCUB. Each spectrum is taken every $5^{\prime}$ in both right ascension and declination. Each spectrum is taken every $5^{\prime}$ in both right ascension and declination. 


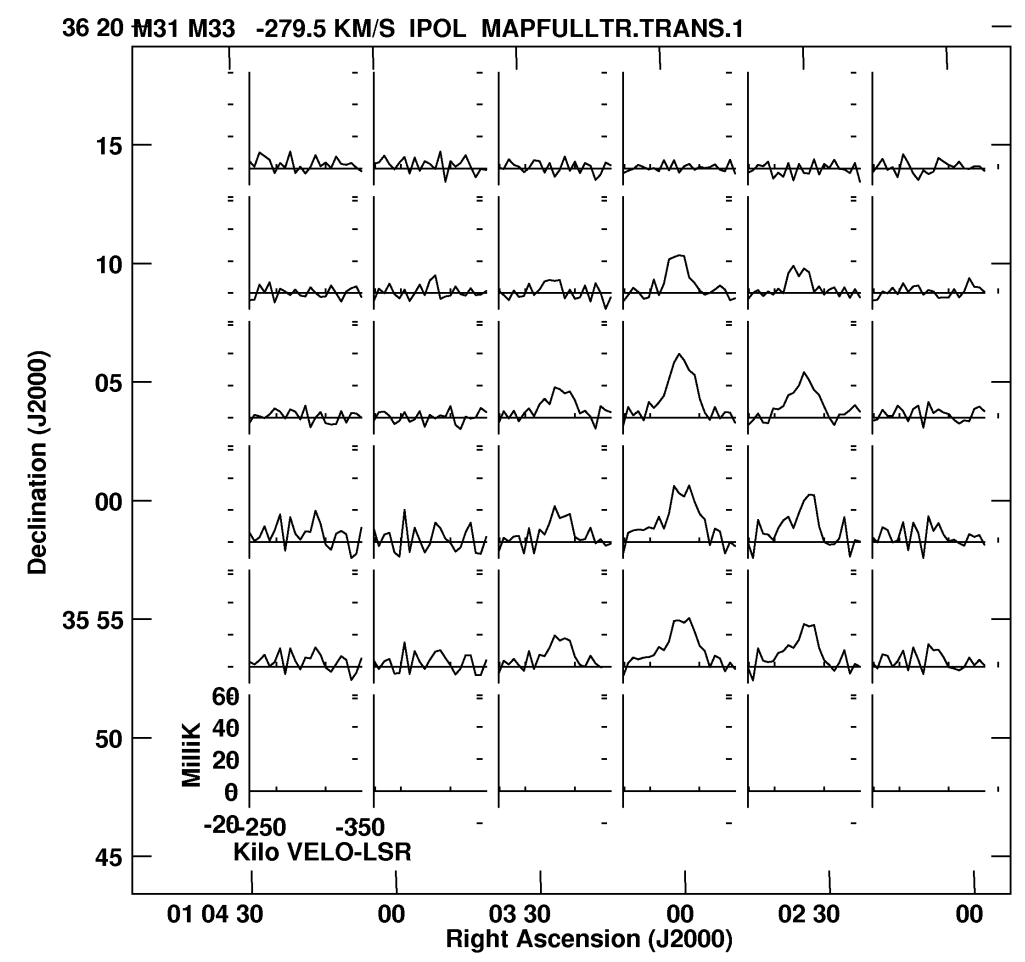

Figure 4.12: H I spectra across cloud 6 using the AIPS task PLCUB. Each spectrum is taken every $5^{\prime}$ in both right ascension and declination.

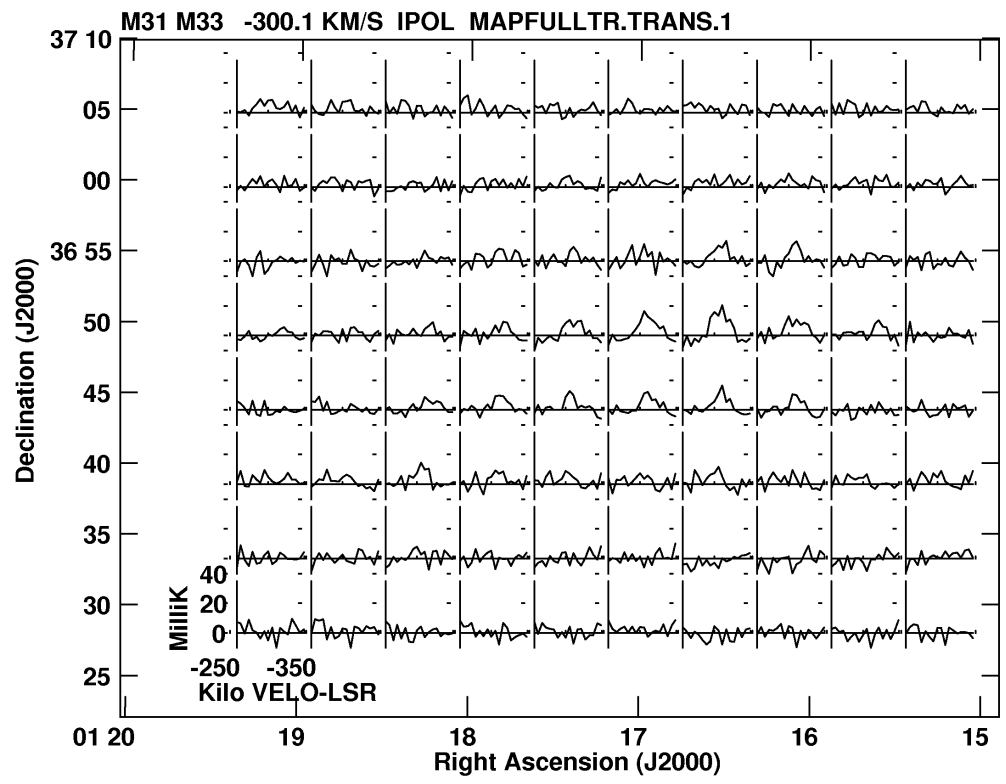

Figure 4.13: H I spectra across cloud 7 using the AIPS task PLCUB. Each spectrum is taken every $5^{\prime}$ in both right ascension and declination. 
large wings instead of isolated spectral lines. All detections except the one at $\mathrm{v}_{\mathrm{LSRK}}$ of $-238 \mathrm{~km} \mathrm{~s}^{-1}$ were usually no more than $40 \mathrm{~km} \mathrm{~s}^{-1}$ away from Milky Way emission. Thus, it is likely that some fraction of the Braun \& Thilker (2004) emission seen to the northwest of M31 might very well be confused with foreground Milky Way gas. Figure 4.1 shows our pointings. It should be noted that this emission has not been corrected for stray radiation, where emission from the side lobes of the telescope can create spectral features. While the contribution of the GBT's side lobes are small, they are extended from the main beam by several degrees (Boothroyd et al., 2011) and thus as one moves closer to a bright source, such as M31, the need to correct for stray emission becomes more important. The stray correction will be done in future work.

\begin{tabular}{c|c|c|c|c|c|c|c|c|c} 
& r.a. & $\delta$ & Detection & Peak & $\begin{array}{c}\text { VLSR } \\
\text { LK }\end{array}$ & $\begin{array}{c}\text { FWHM } \\
\mathrm{km} \mathrm{s}^{-1}\end{array}$ & $\begin{array}{c}\text { RMS } \\
\mathrm{mK}\end{array}$ & $\begin{array}{c}\mathrm{N}_{\text {HI }} \times 10^{17} \mathrm{~cm}^{-2} \\
\text { integrated }( \pm 1 \sigma)\end{array}$ & $\begin{array}{c}\text { Time } \\
\text { minutes }\end{array}$ \\
\hline 1 & $00: 10: 00$ & $+46: 00: 00$ & Yes & 7.3 & -190 & $23 \pm 3.2$ & 3.4 & $3.2 \pm 0.7$ & 84 \\
2 & $00: 10: 00$ & $+47: 00: 00$ & Yes & 25 & -181 & $25.0 \pm 1.5$ & 3.1 & $12 \pm 0.7$ & 80 \\
3 & $00: 30: 00$ & $+45: 00: 00$ & No & - & - & - & 3.3 & - & 80 \\
4 & $00: 15: 00$ & $+46: 00: 00$ & Yes & 13 & -238 & $12.8 \pm 2.0$ & 3.6 & $3.2 \pm 0.7$ & 80 \\
5 & $00: 15: 00$ & $+47: 00: 00$ & No & - & - & - & 3.2 & - & 84 \\
6 & $00: 20: 00$ & $+45: 00: 00$ & MW & - & - & - & 4.0 & - & 56 \\
7 & $00: 20: 00$ & $+47: 00: 00$ & Yes & 22 & -167 & $19.6 \pm 1.2$ & 3.0 & $8.3 \pm 0.6$ & 84 \\
8 & $00: 20: 00$ & $+48: 00: 00$ & No & - & - & - & 3.1 & - & 84 \\
9 & $00: 25: 00$ & $+45: 00: 00$ & MW & - & - & - & 3.6 & - & 70 \\
10 & $00: 25: 00$ & $+47: 00: 00$ & Yes & 8.0 & -193 & $30.0 \pm 2.7$ & 3.1 & $4.6 \pm 0.6$ & 84 \\
11 & $00: 30: 00$ & $+45: 00: 00$ & No & - & - & - & 3.4 & - & 80 \\
12 & $00: 30: 00$ & $+47: 00: 00$ & No & - & - & - & 4.5 & - & 56 \\
13 & $00: 40: 00$ & $+46: 00: 00$ & MW & - & - & - & 3.2 & - & 80 \\
14 & $00: 35: 00$ & $+45: 00: 00$ & MW & - & - & - & 3.2 & - & 84 \\
15 & $00: 35: 00$ & $+47: 00: 00$ & No & - & - & - & 4.4 & - & 56 \\
16 & $00: 40: 00$ & $+45: 00: 00$ & No & - & - & - & 3.6 & - & 80 \\
17 & $00: 40: 00$ & $+46: 00: 00$ & MW & - & - & - & 4.1 & - & 56 \\
18 & $00: 40: 00$ & $+47: 00: 00$ & MW & - & - & - & 3.3 & - & 80 \\
19 & $00: 45: 00$ & $+47: 00: 00$ & Yes & 10 & -182 & $24.5 \pm 3.0$ & 3.1 & $4.7 \pm 0.6$ & 84
\end{tabular}

Table 4.2: Results from our GBT pointings to the northwest of M31. The column density was measured by summing the area under the spectra. 


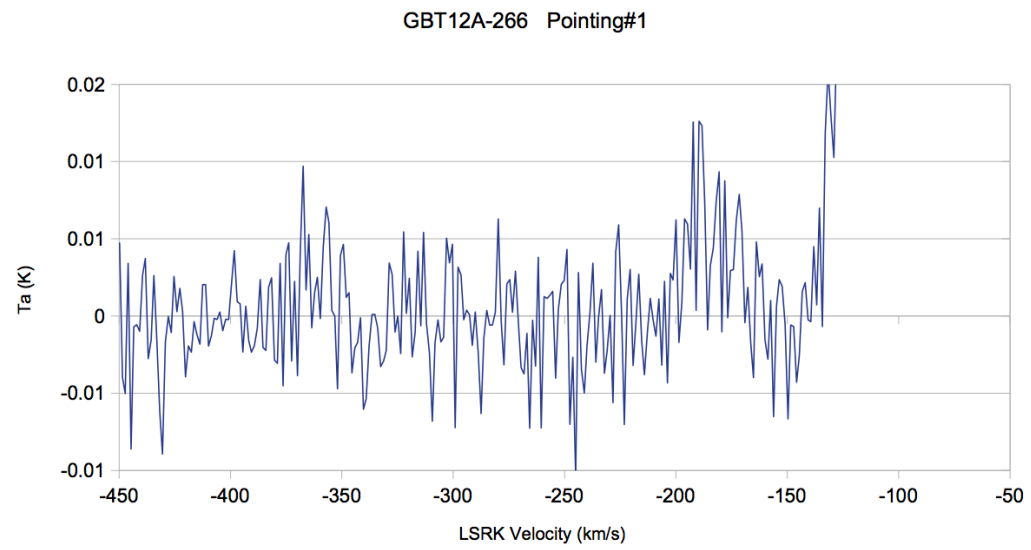

Figure 4.14: $21 \mathrm{~cm}$ spectrum from pointing 1. The $\mathrm{x}$ axis is the LSR velocity in $\mathrm{km} \mathrm{s}^{-1}$. The $\mathrm{y}$ axis is the antenna temperature in $\mathrm{K}$.

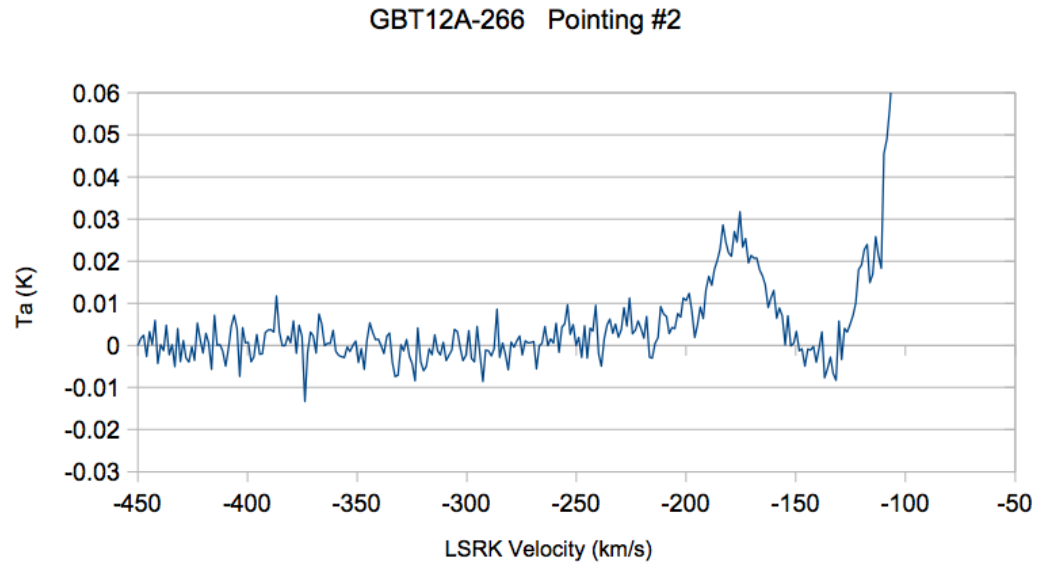

Figure 4.15: $21 \mathrm{~cm}$ spectrum from pointing 2. The $\mathrm{x}$ axis is the LSR velocity in $\mathrm{km} \mathrm{s}^{-1}$. The $\mathrm{y}$ axis is the antenna temperature in $\mathrm{K}$.

The average column densities of our detections are $10^{17.7} \mathrm{~cm}^{-2}$, which is similar to the column densities measured for the M31 clouds. These column densities may not be at the peak of the emission however, given that they come from pointings. Further, the average linewidth (FWHM) of our detections $\sim 23 \mathrm{~km} \mathrm{~s}^{-1}$, which is also similar to the average line width of the M31 clouds $\left(\sim 27 \mathrm{~km} \mathrm{~s}^{-1}\right)$, which suggests that the H I emission may be part of a similar population of objects. 
GBT12A-266 Pointing \#4

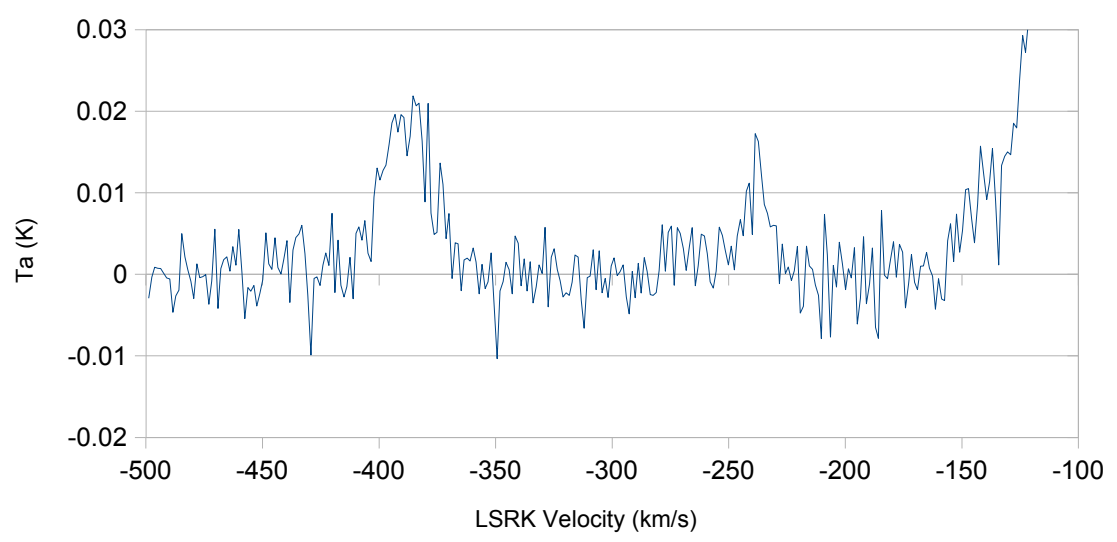

Figure 4.16: $21 \mathrm{~cm}$ spectrum from pointing 4. The $\mathrm{x}$ axis is the LSR velocity in $\mathrm{km} \mathrm{s}^{-1}$. The $\mathrm{y}$ axis is the antenna temperature in $\mathrm{K}$.

GBT12A-266 Pointing \#7

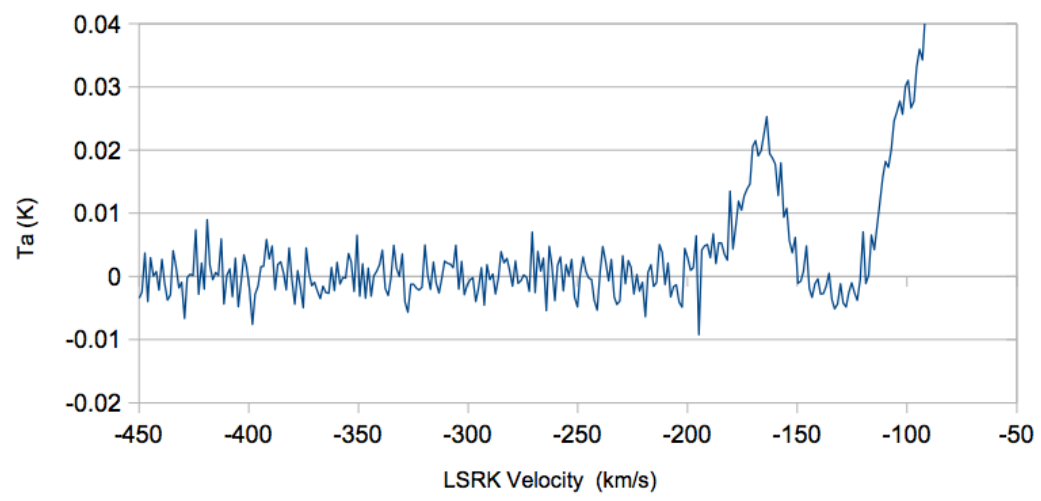

Figure 4.17: $21 \mathrm{~cm}$ spectrum from pointing 7. The $\mathrm{x}$ axis is the LSR velocity in $\mathrm{km} \mathrm{s}^{-1}$. The $\mathrm{y}$ axis is the antenna temperature in $\mathrm{K}$.

\subsection{Discussion}

The M31-M33 emission first detected by Braun \& Thilker (2004) is still in the form of $\mathrm{H}$ i clouds when mapped with the $9^{\prime}$ GBT beam. Extensions appear on some of the clouds. The extension for cloud 1 is either a spatially coincident cloud or is material that has been stripped from it. Cloud 4 shows head-tail structure, but there 


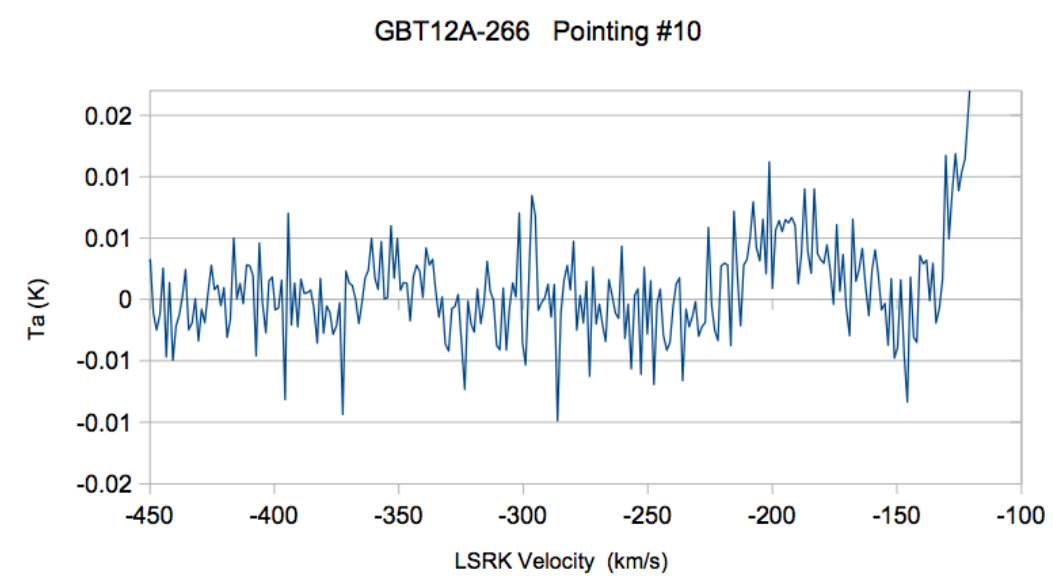

Figure 4.18: $21 \mathrm{~cm}$ spectrum from pointing 10. The $\mathrm{x}$ axis is the LSR velocity in $\mathrm{km} \mathrm{s}^{-1}$. The $\mathrm{y}$ axis is the antenna temperature in $\mathrm{K}$.

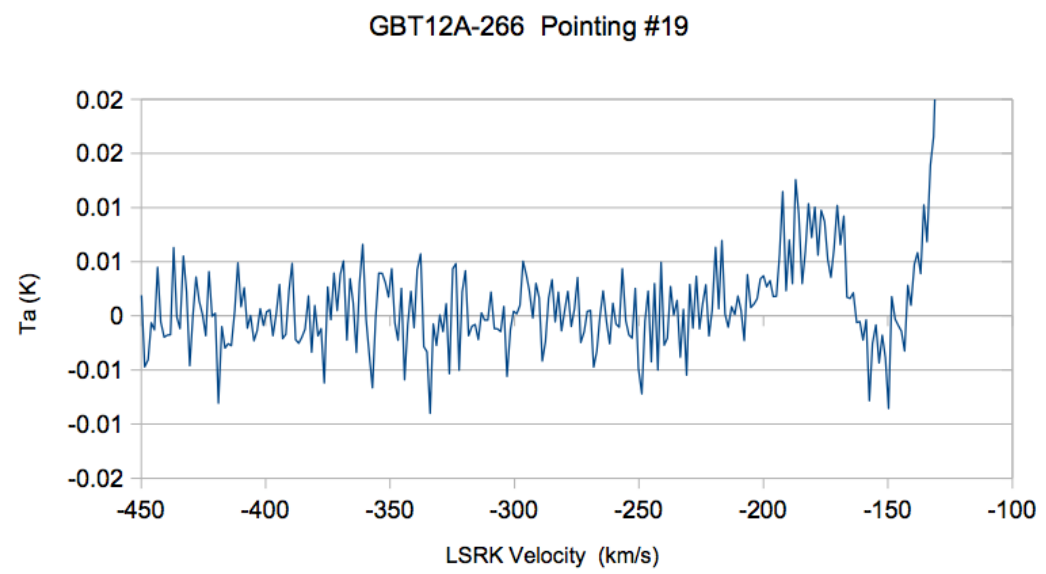

Figure 4.19: $21 \mathrm{~cm}$ spectrum from pointing 19. The $\mathrm{x}$ axis is the LSR velocity in $\mathrm{km} \mathrm{s}^{-1}$. The $\mathrm{y}$ axis is the antenna temperature in $\mathrm{K}$.

is a small velocity gradient along the tail, as with all the other cloud gradients in velocity. The gradients are only a few channels wide and while they are likely real features, we can't draw any firm conclusions from them. The lack of any evidence of line broadening and small velocity gradients lead us to conclude that there is no evidence of an interaction between the clouds and a surrounding medium. Higher velocity resolution observations are needed. 
The basic morphology of the Smith Cloud (Smith, 1963), a high velocity cloud roughly $12 \mathrm{kpc}$ distant (Lockman et al., 2008), is roughly similar to cloud 4 when smoothed to the same linear resolution as our GBT data (Figure 4.20). We specifically used the Smith Cloud data taken with the GBT by Lockman et al. (2008) for the spatial smoothing. We convolved the data cube by an appropriately sized Gaussian that represents the GBT beam at approximately $750 \mathrm{kpc}$. One can see similar velocity structure with gradient of around $20 \mathrm{~km} \mathrm{~s}^{-1}$, similar to cloud 4. Smith's cloud may be part of a larger stream of material that is interacting with the surrounding medium (Lockman, 2012). Given these similarities, cloud 4 may be interacting with a surrounding medium, although this is not conclusive.

Recent advancements to our understanding of the proper motion of M31 (Sohn et al., 2012) predict that the Milky Way, M31 and M33 may undergo several interactions within the next few billion years (van der Marel et al., 2012). Least action based modeling (e.g. Peebles, 1995) using the revised measurements show that M31 and M33 may have had an interaction in the past, as well as with IC10 (Figure 4.21). The crossing times of the clouds, however, are on the order of $\sim 100 \mathrm{Myr}$ and are too short for the clouds to be due to an interaction thought to have occurred a few Gyr ago (Bekki, 2008; McConnachie et al., 2009). The warm-hot intergalactic medium may supply the necessary pressure to allow the clouds to persist for longer periods of time if the WHIM's densities were at least $10^{-7} \mathrm{~cm}^{-3}$, which is consistent with predictions by Fukugita \& Peebles (2004).

We also have updated the Tully-Fisher plot from Chapter 3 with the revised cloud masses. While the clouds move closer to the relation, they still lie below it. 

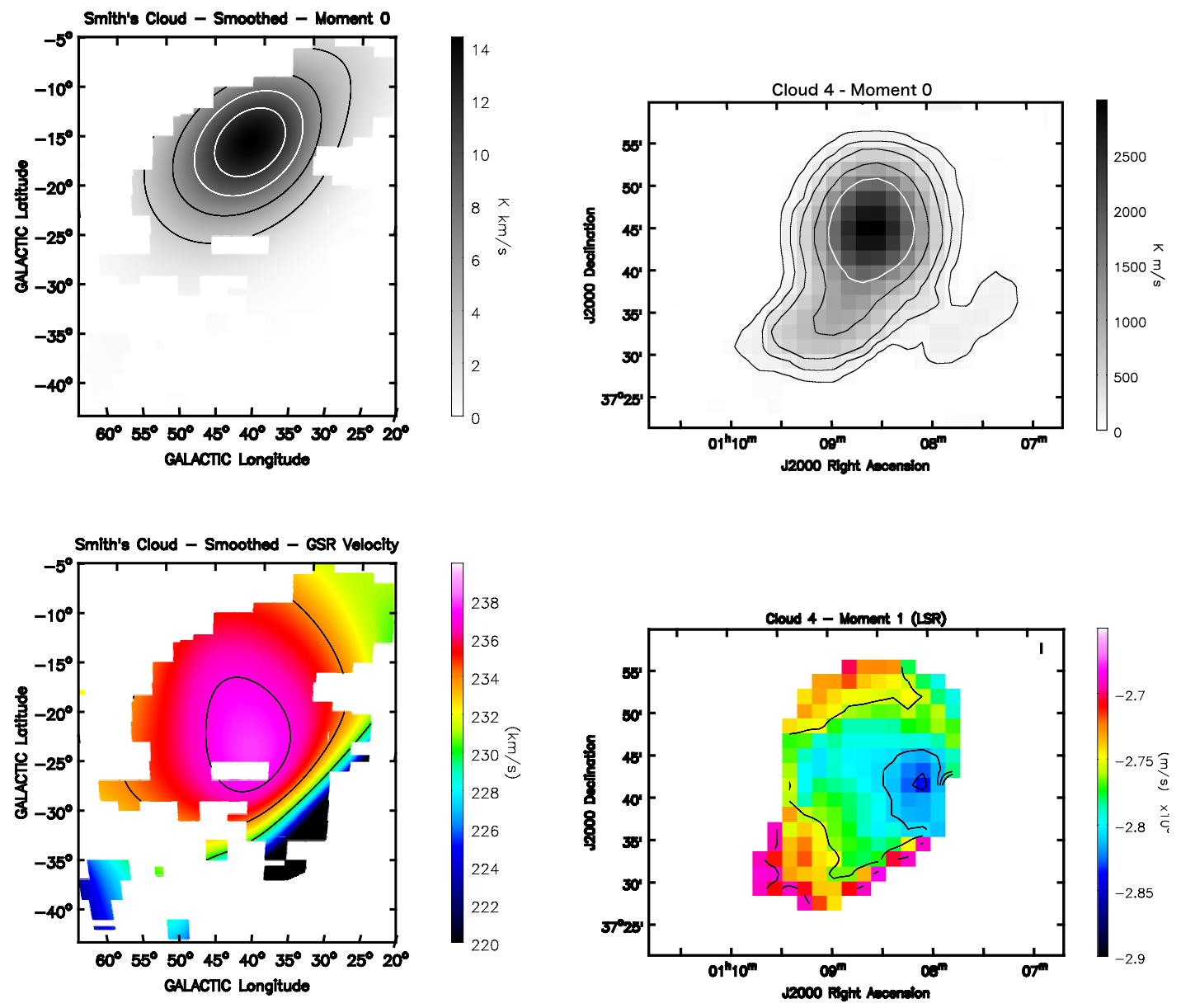

Figure 4.20: The Smith cloud smoothed to the same linear resolution as the M31 clouds. Upper left: The moment-0 map for Smith's cloud, with contours drawn at 3, 6, 9 and $12 \mathrm{~K} \mathrm{~km} \mathrm{~s}^{-1}$. Lower left: Smith's cloud velocity field in the galactic standard of rest (GSR) frame, which corrects for the rotation of the Milky Way. The contours are for GSR $=230,234,237 \mathrm{~km} \mathrm{~s}^{-1}$. Upper right: Cloud 4, with the same contours from Fig. 4.2. Lower right: Cloud 4 velocity field in the LSRK frame. The contours are for $-285,-280,-275$ and $-270 \mathrm{~km} \mathrm{~s}^{-1}$.

They still remain more highly clustered than the other Local Group objects with no clear connection to any of the dwarf galaxies.

Given the results from our analysis of the 400 hour survey, when compared to the 250 hours of data (Chapter 3), we conclude that the M31-M33 H i clouds are still likely to be condensations from an intergalactic filament, although a tidal feature can not be ruled out. The major difference between the new results and the preliminary 


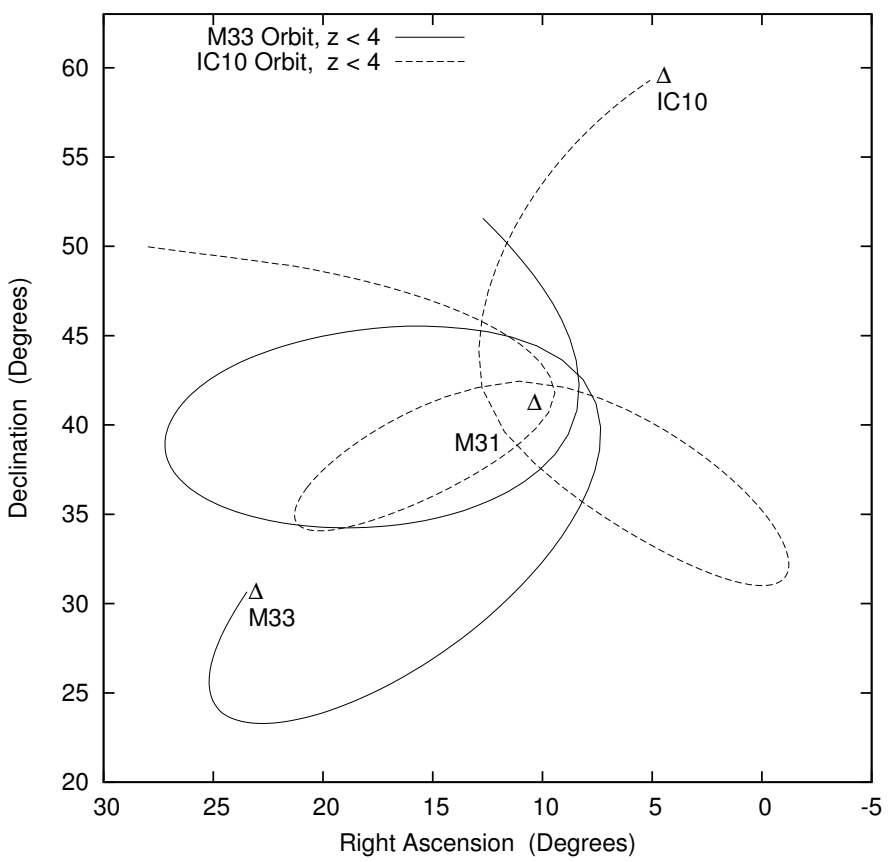

Figure 4.21: Modeling of the M31-M33-IC10 system using the revised proper motion measurement of M31 and numerical action modeling. Potential interactions between M31, M33 and IC 10 can clearly be seen. Data for the figure provided by Edward Shaya.

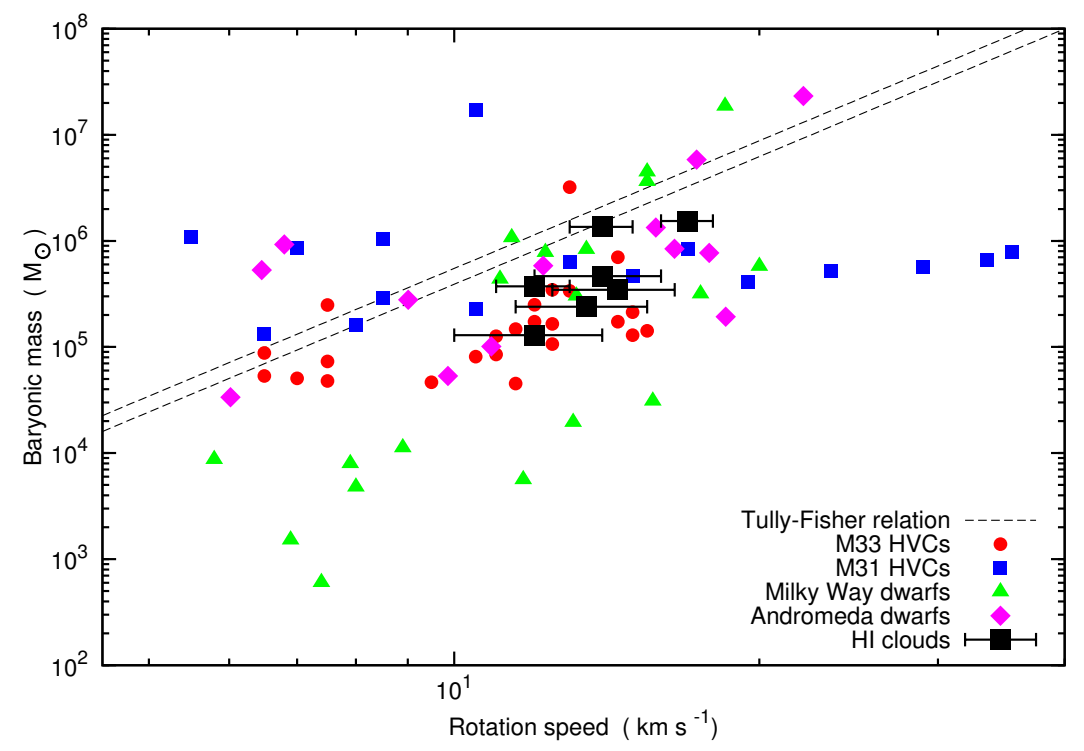

Figure 4.22: An updated plot of the H I clouds and other Local Group objects against the TullyFisher relation. The procedure for producing the plot is the same as for Chapter 3.

ones are that all of the $\mathrm{H}$ i discovered in the Westerbork survey appears to be in the form of H i clouds. 
Roughly a third of our pointings to the northwest of M31 have Milky Way emission at velocities similar to our detections. Thus, it is likely that some of the emission seen in Braun \& Thilker (2004) is foreground emission from the Galaxy. Nevertheless, we can still attempt to draw some conclusions of the diffuse H i we did detect.

First, the average linewidth of the spectra are nearly the same as the average linewidth of M31's HVCs seen to the northwest ( $21 \mathrm{~km} \mathrm{~s}^{-1}$; Westmeier et al., 2008), suggesting that the emission might be part of the HVC population.

Second, we plot the local group standard of rest (LGSR) velocity of the detections versus angular distance from M31, which accounts for the Milky Way's motion with respect to the Local Group barycenter, as described and conducted in Chapter 3. The new detections, along with our original clouds and other Local Group objects are shown in Figure 4.23. Our detections are at about the same velocities and only lie slightly further from M31 than some of its HVCs. Thus, it is very likely that these detections are either confusion with foreground Milky Way H I, or are associated with the HVC population of M31.

\subsection{Conclusions}

To summarize, we find that the conclusions from the 250 hour survey still hold. The M31-M33 clouds are likely condensations from an intergalactic filament. The external pressure of the WHIM could allow the clouds to persist for longer periods after a tidal interaction, but there is no direct evidence of line broadening or conclusive 


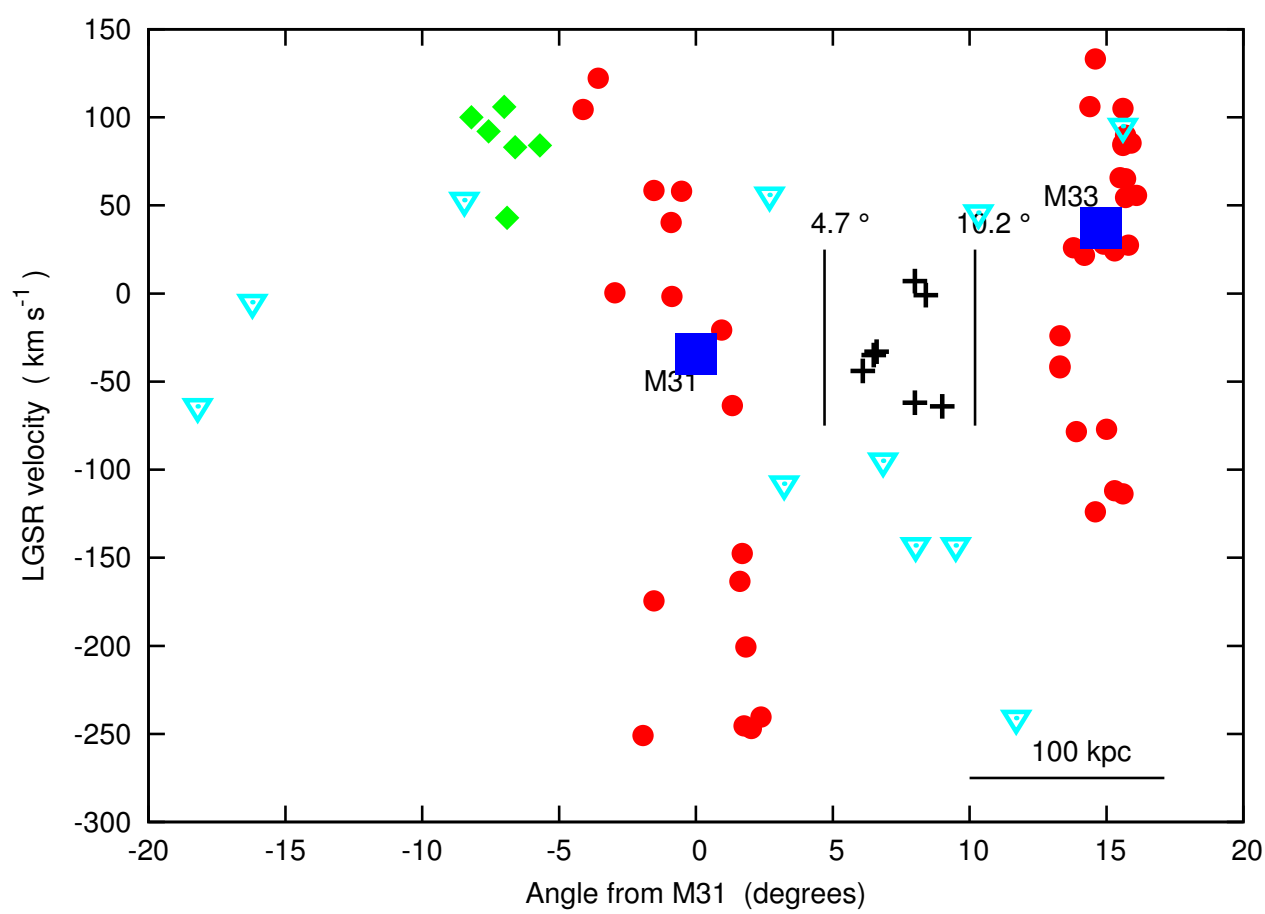

Figure 4.23: The LGSR velocity versus angular distance from M31 for both M31's and M33's high velocity clouds (red circles), the clouds we detected from our 400 hour survey (black crosses), our new detections to the northwest of M31 (green diamonds) and the Andromeda dwarfs (aqua triangles). The left most triangle is IC 10. LGSR velocities were taken from NED.

evidence of velocity gradients for the clouds. Cloud 4 appears similar to Smith's cloud which suggests that it may be on a similar evolutionary path, but given the resolution of the data, it is not conclusive evidence of an interaction with the surrounding medium. Higher resolution observations are needed.

Our pointings to the northwest of M31 did detect faint $\mathrm{H}$ i emission there, but it is likely the result of foreground emission from the Milky Way, or is part of the HVC population of M31. Mapping of this area has begun and will hopefully shed further light on what the origins of this gas are. We are also planning on re-reducing all of our survey data but at a higher velocity resolution. While we will lose sensitivity in the process, given that our clouds are on the order of 10 times brighter than we expected 
the emission to be, we should still be able see emission and do a more thorough study of the kinematics. 


\section{Chapter 5}

\section{High and Intermediate Velocity $\mathrm{H}_{\mathrm{I}}$ in the Southern Sky}

We present southern sky maps of anomalous velocity H I from the Galactic AllSky Survey. This survey is one of the most sensitive Hi surveys of the southern sky to date, with high spatial and angular resolution. We examine the basic properties of this material at both high and intermediate velocities and produce the most detailed maps of the high velocity and intermediate velocity gas to date, the latter of which has never before been published for the southern sky. We investigate the sky covering fraction and column density distribution of this gas. We also examine and discuss several distinct features seen in the maps. Some of this gas, the Smith Cloud in particular, may be in the final stages of accretion onto the Galaxy, representing one possible outcome of the fate of the M31 - M33 H i clouds (Wolfe et al., 2013).

\subsection{Background}

The existence of anomalous velocity gas around our Milky Way has been known for over 50 years. van Woerden et al. (1957) suggested that clouds of H i could condense from the hot Galactic halo (Spitzer, 1956) and fall toward the Milky Way at high velocities. Muller et al. (1963) discovered such clouds of $\mathrm{H}_{\mathrm{I}}$ with $\left|\mathrm{v}_{\mathrm{LSR}}\right| \geq 100 \mathrm{~km} \mathrm{~s}^{-1}$, the so-called High Velocity Clouds (HVCs). 
These clouds likely represent a variety of phenomena. A possible origin is that they are primordial gas clouds that are currently being accreted by the Milky Way (Oort, 1966). Alternatively, HVCs may be debris from interactions between dwarf galaxies and the Milky Way, such as the Magellanic Stream (Bland-Hawthorn et al., 1998; Bregman, 2004), which is a large tidal stream of gas over $200^{\circ}$ long on the sky that is a result of tidal stripping between the Magellanic clouds and the Milky Way. Another possibility is the so-called Galactic fountain model, in which gas clouds are formed when gas circulates out of the Galactic plane into the halo by supernovae, condenses and then falls back onto the disk (e.g. Bregman, 1980; Shapiro \& Field, 1976); intermediate velocity clouds (IVCs) are thought to fall primarily into the last category of objects. The metallicities of IVCs that have been measured are at or near solar values (Wakker, 2001); gas originating from the Galactic disk should have higher metallicities than gas from the intergalactic medium (IGM) or the Local Group due to enrichment from the interstellar medium. This with their low velocities suggests that IVCs most likely originate from the Galactic disk. Most large HVC complexes tend to have much lower metallicity, on the order of 10\% solar (Wakker et al., 1999; Collins et al., 2007). This, with their higher velocities, suggests that the large HVCs complexes likely originate outside the Galactic disk.

No large HVC complexes exist in the southern sky, aside from the Magellanic Clouds and Streams. Furthermore, no comprehensive map of IVC emission in the southern sky has been published. To help further our understanding of the HVC and IVC phenomena, a thorough exploration of their distribution across the southern sky is necessary. The Leiden/Argentine/Bonn Galactic H I Survey (LAB) covered 
the entire sky with an effective angular resolution of $\sim 1^{\circ}$ and velocity resolution of $\sim 1 \mathrm{~km} \mathrm{~s}^{-1}$ (Kalberla et al., 2005). It is known, however, that physical structure exists for HVCs on scales smaller than 30' (e.g. Brüns et al., 2005; Westmeier et al., 2005). The H I Parkes All-Sky Survey (Barnes et al., 2001; Putman et al., 2002, HIPASS) provides $\sim 15^{\prime}$ resolution and high sensitivity in the south, but with velocity channel spacings of $\sim 24 \mathrm{~km} \mathrm{~s}^{-1}$, which is too wide to study the internal kinematics of many HVCs and IVCs with can have linewidths as small as a few $\mathrm{km} \mathrm{s}^{-1}$ (e.g. Westmeier et al., 2005). A sensitive H I survey with the adequate spatial and velocity resolution is needed. The Parkes Galactic All-Sky Survey (GASS) was specifically designed to provide a high velocity resolution, high angular resolution survey of $\mathrm{H}$ I covering the entire southern sky. We therefore use the GASS data to study both the global properties of the HVC and IVC gas and generate southern sky maps of the emission.

Separating $21 \mathrm{~cm} \mathrm{HVC}$ and IVC emission from that of the Milky Way is not a straightforward process. Historically, to identify high velocity gas, astronomers have selected emission based on its velocity relative to the kinematic local standard of rest (LSRK), $\mathrm{v}_{\mathrm{LSR}}$. Using this, high-velocity clouds (HVCs) are typically defined with $\left|\mathrm{v}_{\mathrm{LSR}}\right| \gtrsim 90 \mathrm{~km} \mathrm{~s}^{-1}$ and intermediate velocity clouds (IVCs) have $40 \lesssim\left|\mathrm{v}_{\mathrm{LSR}}\right| \lesssim$ $90 \mathrm{~km} \mathrm{~s}^{-1}$ (e.g. Wakker, 2004). However, these limits are not rigorously defined. If one is to understand the anomalous velocity gas in detail, a different method of filtering out Milky Way emission is preferred. One can define a deviation velocity, $\mathrm{v}_{\text {dev }}$ (Wakker, 1990), which is the difference between the anomalous velocity emission and the maximum velocity allowed by a model of the $\mathrm{H}$ I distribution and Galactic rotation along the given sightline (van Woerden et al., 2004). This helps to remove 
Milky Way emission that at some positions, particularly near the Galactic plane, can have $\mathrm{v}_{\mathrm{LSR}}$ beyond the HVC limits. For this work, clouds with $\left|\mathrm{v}_{\mathrm{dev}}\right| \geq 90 \mathrm{~km} \mathrm{~s}^{-1}$ are considered to be HVCs, while clouds that have $\left|\mathrm{v}_{\mathrm{dev}}\right|=30-90 \mathrm{~km} \mathrm{~s}^{-1}$ are the IVCs.

\subsection{The Galactic All-Sky Survey}

The Galactic All-Sky Survey, or GASS, was designed to optimally study the interaction between the halo and the disk of the Milky Way, along with the structure of the Milky Way and the Magellanic system (McClure-Griffiths et al., 2009). The $21 \mathrm{~cm}$ data were collected with the multi-beam receiver on the Parkes $64 \mathrm{~m}$ radio telescope with a central beamwidth of $14.0^{\prime}$ and a channel width of $1.0 \mathrm{~km} \mathrm{~s}^{-1}$. The survey reached a $3 \sigma$ sensitivity limit of $\mathrm{N}_{\mathrm{HI}}=1.6 \times 10^{18} \mathrm{~cm}^{-2}$ over a $30 \mathrm{~km} \mathrm{~s}^{-1}$ linewidth. Detailed information about the survey, reduction and first data release can be found in McClure-Griffiths et al. (2009). The data used in this paper are from the second data release, which has better baselines and corrections for stray radiation (Kalberla et al., 2010). The GASS data on which this work is based was partitioned into 20 separate data cubes, each covering the southern sky and spanning roughly $50 \mathrm{~km} \mathrm{~s}^{-1}$ in velocity for $\left|\mathrm{v}_{\mathrm{LSR}}\right| \leq 500 \mathrm{~km} \mathrm{~s}^{-1}$.

It should be noted that Moss et al. (2013) have recently conducted an analysis of the GASS data to produce a catalogue of the detected, individual anomalous velocity clouds. While that work focuses on the specifics of the clouds, this work focuses primarily on the overall global properties and features seen in the GASS data. We will, however, compare our results with those of Moss et al. (2013) where appropriate. 


\subsection{Blanking Milky Way Emission}

To create synthetic spectra that represent emission from the Milky Way, we use the model by de Heij et al. (2002). The brightness temperature, from the equation of radiative transfer, assuming no contribution from background continuum is

$$
\mathrm{T}_{\mathrm{B}}(\mathrm{v})=\mathrm{T}_{\mathrm{K}} \cdot\left(1-\mathrm{e}^{-\tau_{\mathrm{v}}}\right) .
$$

$\mathrm{T}_{\mathrm{B}}$ is the brightness temperature in $\mathrm{K} . \mathrm{T}_{\mathrm{K}}$ is the kinetic temperature, assumed to be $100 \mathrm{~K}$ (de Heij et al., 2002). $\tau_{\mathrm{v}}$ is the optical depth of the gas and can be calculated (e.g. Spitzer, 1978) as a function of velocity using

$$
\mathrm{d} \tau\left(\frac{\mathrm{v}}{\mathrm{km} \mathrm{s}^{-1}}\right)=5.487 \times 10^{-19} \cdot \frac{\mathrm{n}_{\mathrm{HI}}\left(\mathrm{cm}^{-3}\right)}{\mathrm{T}_{\mathrm{K}}(\mathrm{K})} \cdot \Phi\left(\frac{\mathrm{v}}{\mathrm{km} \mathrm{s}^{-1}}\right) \cdot \mathrm{ds}(\mathrm{cm}) .
$$

ds is the path length, which is our chosen grid size of $50 \mathrm{pc} . \mathrm{n}_{\mathrm{HI}}$ is the number density of $\mathrm{H}$ r atoms and $\Phi$ is the spectral line shape. It is assumed the Milky Way has a flat rotation curve at all galactocentric radii $\mathrm{R}$ with $\mathrm{v}_{\mathrm{rot}}=220 \mathrm{~km} \mathrm{~s}^{-1}$ and $\sigma_{\mathrm{v}}=20 \mathrm{~km} \mathrm{~s}^{-1}$ (de Heij et al., 2002) so that $\Phi$ can be written as

$$
\Phi(\mathrm{v})=\frac{1}{\sigma_{\mathrm{v}} \sqrt{2 \pi}} \mathrm{e}^{-\frac{\left(\mathrm{v}-\mathrm{v}_{\mathrm{rot}}\right)^{2}}{2 \sigma_{\mathrm{v}}}} .
$$

Thus, for a single velocity channel, the optical depth becomes

$$
\tau_{\mathrm{v}}=\frac{33.52 \cdot \mathrm{n}_{\mathrm{HI}}}{\mathrm{T}_{\mathrm{K}} \cdot \sigma_{\mathrm{v}}} \cdot \mathrm{e}^{-\frac{\left(\mathrm{v}-\mathrm{v}_{\mathrm{rot}}\right)^{2}}{2 \sigma_{\mathrm{v}}^{2}}} .
$$


For $\mathrm{n}_{\mathrm{HI}}$, the de Heij et al. (2002) model treats the Milky Way H I as a thin disk with a constant central density, $\mathrm{n}_{\mathrm{HI}, \mathrm{o}}=0.35 \mathrm{~cm}^{-3}$ within $\mathrm{R}=11.5 \mathrm{kpc}$. Beyond $11.5 \mathrm{kpc}$, the midplane density exponentially decreases in the radial direction with a scale length of $3 \mathrm{kpc}$. The motivation for de Heij et al. (2002) to choose these parameters was so that their model better resembled the data from the Leiden/Dwingeloo Survey (Hartmann \& Burton, 1997)

$$
\mathrm{n}_{\mathrm{HI}}(\mathrm{R}>11.5 \mathrm{kpc})=\mathrm{n}_{\mathrm{HI}, \mathrm{o}} \cdot \mathrm{e}^{-\frac{\mathrm{R}-11.5 \mathrm{kpc}}{3 \mathrm{kpc}}} .
$$

In the vertical direction, the gas density follows a Gaussian distribution to account for the extended $\mathrm{H}$ I disk. For $\mathrm{R} \leq 11.5 \mathrm{kpc}$, we have

$$
\mathrm{n}_{\mathrm{HI}}=\mathrm{n}_{\mathrm{HI}, \mathrm{o}} \cdot \mathrm{e}^{-\frac{\left(\mathrm{z}-\mathrm{z}_{0}\right)^{2}}{2 \sigma_{\mathrm{z}}^{2}}},
$$

where $\sigma_{\mathrm{z}}=180 \mathrm{pc}$, and $\mathrm{z}_{0}=0 \mathrm{pc}$ (de Heij et al., 2002). For $\mathrm{R}>11.5 \mathrm{kpc}$, we have

$$
\begin{gathered}
\frac{\sigma_{\mathrm{z}}[\mathrm{R}>11.5 \mathrm{kpc}]}{\mathrm{pc}}=180+80 \cdot(\mathrm{R}-11.5 \mathrm{kpc}) \\
\frac{\mathrm{z}_{0}[\mathrm{R}>11.5 \mathrm{kpc}]}{\mathrm{pc}}=\frac{\mathrm{R}-11.5}{6} \cdot \sin \phi+\left(\frac{\mathrm{R}-11.5}{6}\right)^{2} \cdot(1-2 \cos \phi),
\end{gathered}
$$

to account for the warp in the Galactic disk (Voskes \& Burton, 1999; Binney \& Merrifield, 1998). $\phi$ is the cylindrical Galactic azimuth centered on the Galactic center, where $\phi=180^{\circ}$ is the direction toward the Sun. 
Using this model, we identify the most positive and negative $\mathrm{v}_{\mathrm{LSR}}$ for $\mathrm{H}_{\mathrm{I}}$ in the model with $\mathrm{T}_{\mathrm{B}} \geq 0.5 \mathrm{~K}$ at every position on the sky and generate maps of the maximum and minimum allowed velocities $\mathrm{v}_{\max }$ and $\mathrm{v}_{\min }$ that are due to Milky Way rotation. These maps were then re-gridded to a zenith-equal-area (ZEA) projection. This projection allows one to see the entire southern hemisphere with no large spatial gaps that would be present in other projections. Each pixel in this projection also has the same area, making a basic analysis of the maps simpler. Because the GASS data are for declinations $\delta \leq 1^{\circ}$, a blanking mask was applied to the $\mathrm{v}_{\max }$ and $\mathrm{v}_{\min }$ maps to blank regions above one degree declination (Figure 5.1). Deviation velocity is defined as: $\mathrm{v}_{\mathrm{dev}}=\mid \mathrm{v}_{\mathrm{LSR}}-\mathrm{v}_{\max }$ OR min $\mid$. Pixels in the cubes whose LSR values gave $\mathrm{v}_{\mathrm{dev}}$ outside of our defined ranges were blanked, resulting in cubes free of Milky Way emission, but still in the LSR velocity frame. This process of blanking in LSR velocity was done separately for the high velocity and intermediate velocity cases. An example of the blanking for the high velocity case is shown in Figure 5.2.

It should be noted that the maximum and minimum velocity limits are dependent upon the choice of the model one uses for the Milky Way. The Kalberla \& Dedes (2008) model for instance uses different scale heights for the disk and empirically fits their functions to survey data over several areas of the Galaxy. Thus, choice of a different model will likely include or exclude more $\mathrm{H}$ i emission. The most likely affected regions of the southern sky would be near the Galactic warp and flare, as well as near the center of the Magellanic Stream, where the H i emission lies at or near Galactic velocities. This should mostly affect IVCs as opposed to HVCs since they also lie closer to Galactic velocities. 

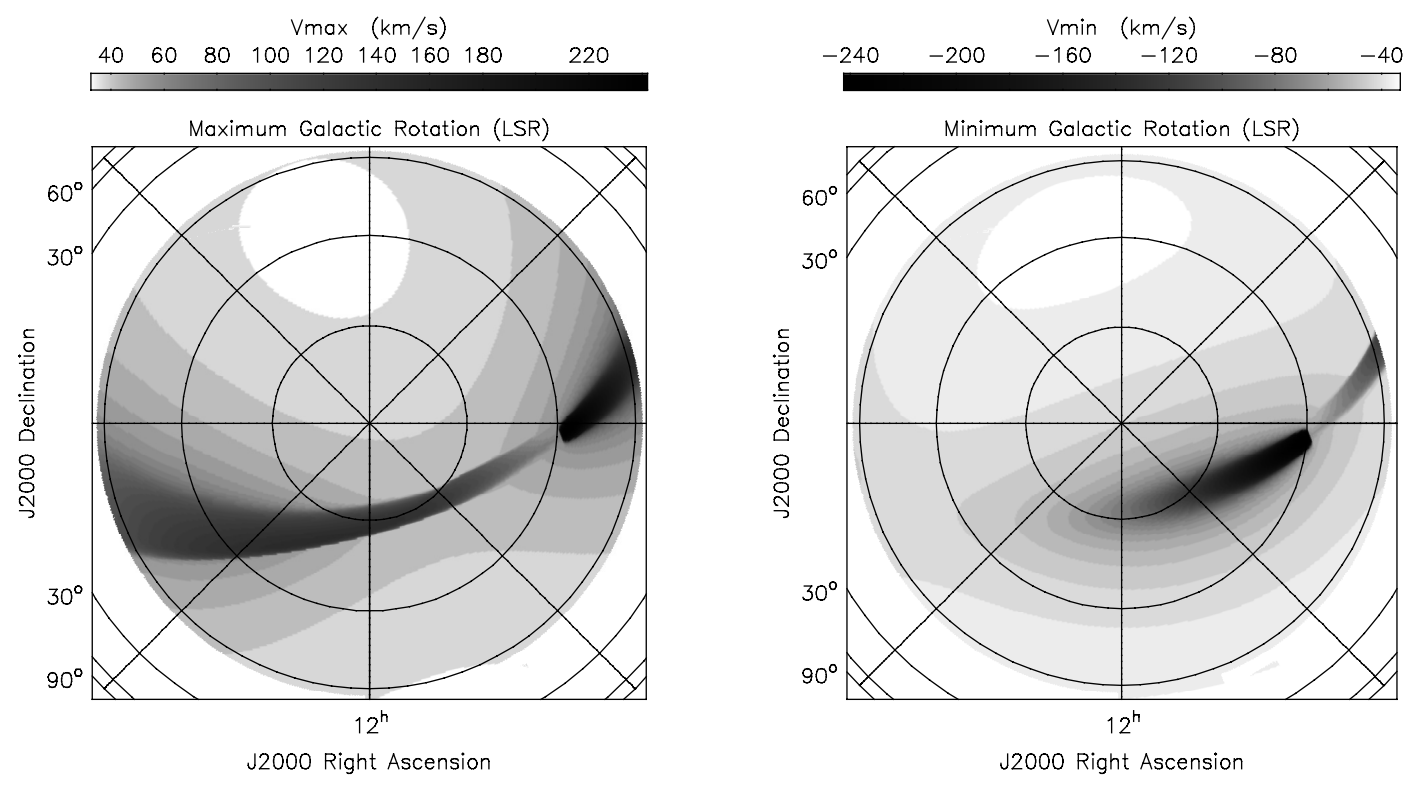

Figure 5.1: Maps of the maximum (left) and minimum (right) H I velocity due to Milky Way rotation in the LSR frame using the model by de Heij et al. (2002). The maps have been masked to match the sky coverage of GASS $\left(\delta \leq 1^{\circ}\right)$.

\subsection{Creating Southern Sky Maps}

\subsubsection{Calculating the Column Density}

To calculate the Hı column density, assuming optically thin Hi emission, one first needs the total $\mathrm{H}$ I intensity, or moment- 0 . The total intensity is simply the brightness temperature integrated over the velocity range of the emission

$$
\mathrm{M} \oslash=\sum_{\mathrm{v}_{\mathrm{i}}} \mathrm{T}_{\mathrm{B}}\left(\mathrm{v}_{\mathrm{i}}\right) \cdot \Delta \mathrm{v},
$$

where we sum over the velocity channels $\mathrm{v}_{\mathrm{i}}$. $\mathrm{T}_{\mathrm{B}}$ is the brightness temperature in $\mathrm{K}$ and $\Delta \mathrm{v}$ is the channel width in $\mathrm{km} \mathrm{s}^{-1}$. The intensity maps were calculated using only brightness temperatures at least $3 \sigma$ above the spectral noise. Because the spectral 

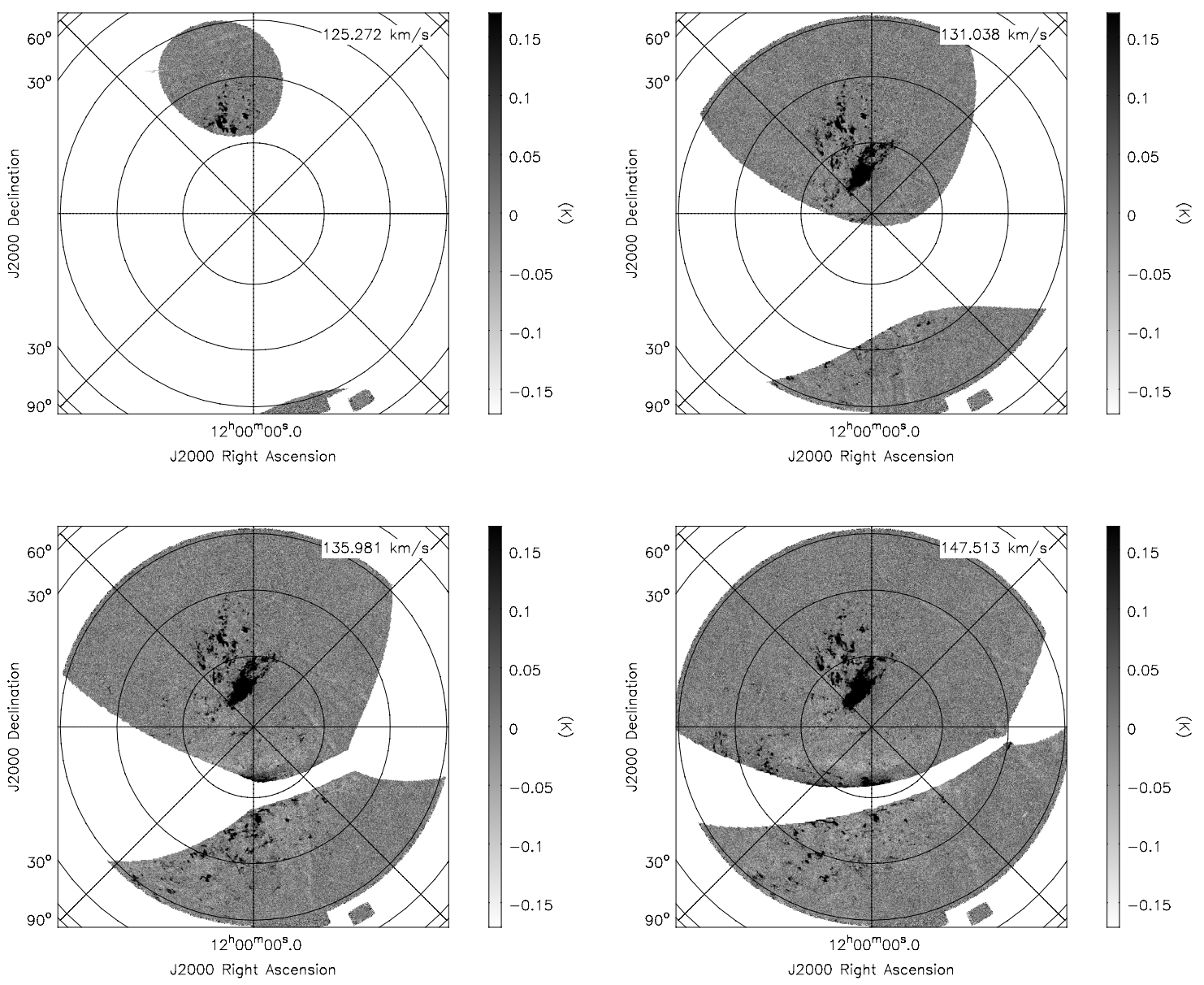

Figure 5.2: Channel maps illustrating the blanking of Milky Way emission in velocity. The blanking here is for the case of high velocity gas. Velocities are with respect to the Local Standard of Rest (LSRK).

noise varies spatially across the GASS data, we took the first several channels of the GASS data from the second data release, $\sim 30 \mathrm{~km} \mathrm{~s}^{-1}$ worth, that were free of emission and calculated the root-mean-square (RMS) value of the spectra across the survey area, allowing us to create an RMS map. This map was then used to blank emission below the $3 \sigma$ noise limit to account for the spatial variations in noise across the survey area. Since the total intensity is a sum over the velocity range of the emission, the intensity maps for each sub-cube $\mathrm{n}$ of the GASS data, $\mathrm{M} \oslash_{\mathrm{n}}$, can simply 
be added together to give the total $\mathrm{H}$ i intensity over all $\mathrm{N}$ cubes

$$
\mathrm{M} \oslash=\sum_{\mathrm{n}=1}^{\mathrm{N}} \mathrm{M} \oslash_{\mathrm{n}}
$$

To calculate the column density, we can use Equation B.21

$$
\mathrm{N}_{\mathrm{HI}}=1.822 \times 10^{18} \mathrm{~cm}^{-2} \int_{\text {line }} \mathrm{T}_{\mathrm{B}}(\mathrm{v}) \cdot \mathrm{dv} \Rightarrow 1.822 \times 10^{18} \mathrm{~cm}^{-2} \cdot \sum_{\mathrm{v}_{\mathrm{i}}} \mathrm{T}_{\mathrm{B}}\left(\mathrm{v}_{\mathrm{i}}\right) \cdot \Delta \mathrm{v}
$$

or

$$
\mathrm{N}_{\mathrm{HI}}=1.822 \times 10^{18} \mathrm{~cm}^{-2} \cdot \mathrm{M} \oslash
$$

where $\mathrm{N}_{\mathrm{HI}}$ is the $\mathrm{H}$ I column density in units of $\mathrm{cm}^{-2}$. The maps for the column density of the high velocity and intermediate velocity gas are shown in Figures 5.3 and 5.4 respectively. The combined data for the HVC and IVC case is shown in Figure 5.5. We note that the maps we generate and those developed by Moss et al. (2013), who used the same Milky Way model to blank Galactic emission, are very similar. We also show a two tone color map to distinguish HVC from IVC material where both populations overlap (Figure 5.6).

\subsubsection{Creating a Map of the Deviation Velocity}

Since the cubes were still in the LSR frame, we first needed to calculate the LSR velocity field from the blanked data cubes. A simple method would be to calculate the moment-1, or intensity weighted velocity for this. This is not suitable for producing all sky maps, given that two different sources of emission might overlap on the sky, 


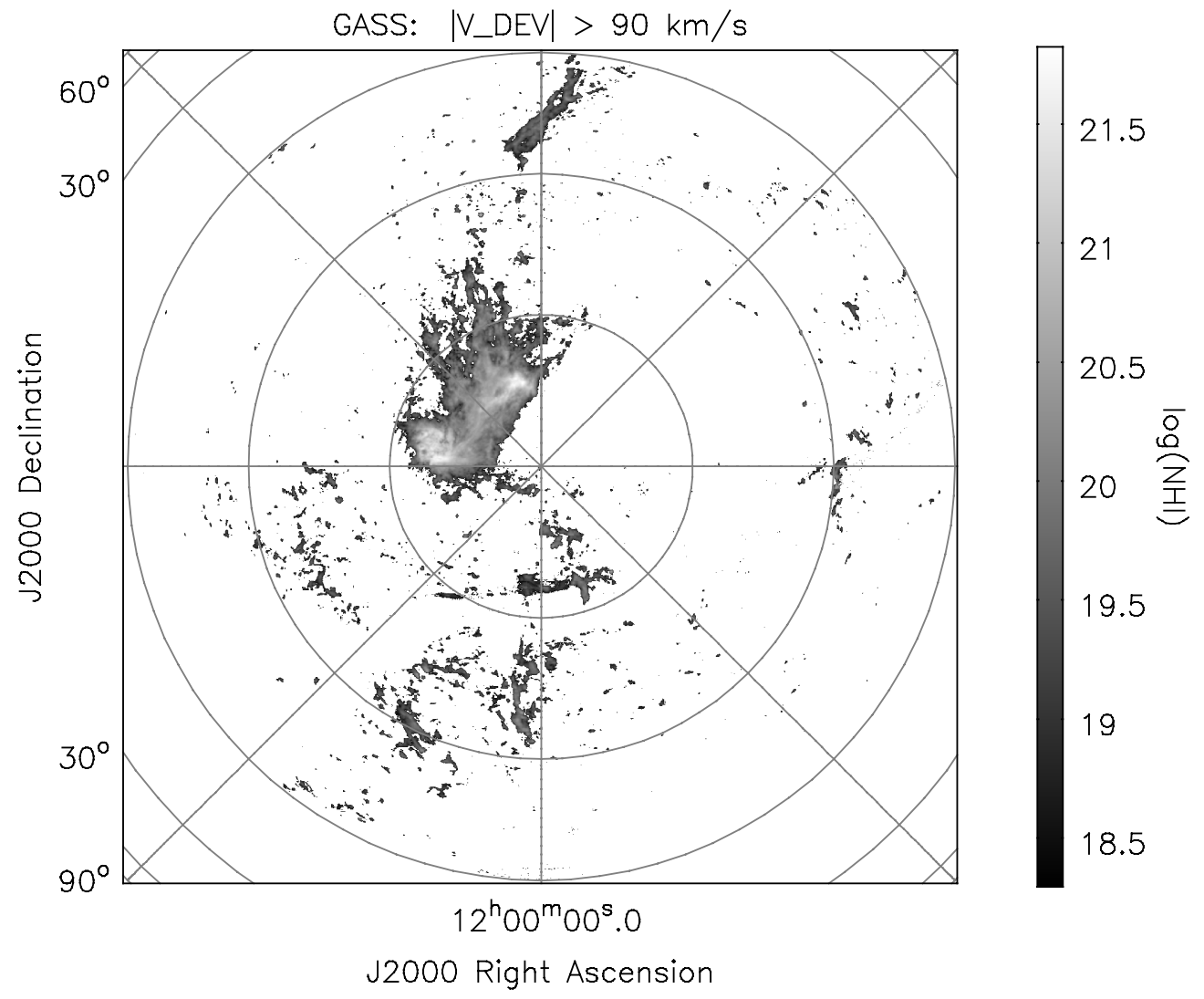

Figure 5.3: Column density map of high velocity gas as seen with GASS $\left(\left|v_{d e v}\right| \geq 90 \mathrm{~km} \mathrm{~s}^{-1}\right)$

but may not overlap in velocity. The resulting velocity would be an average of the two velocities. Instead, we chose to simply calculate the velocity for the highest brightness temperature peak. Specifically, we found the velocity at which the brightness temperature peaked in each of the 20 sub-cubes after the blanking was applied. We then compared the peak temperature at each pixel across all 20 sub-cubes, keeping only the brightest peak and the velocity associated with that peak. This method was done for the HVCs and IVCs separately. It should be noted that this method has the drawback of only showing the brightest emission for a given point on the sky.

To calculate the deviation velocity, we took the difference between the LSR velocity fields and either the maximum or minimum velocity maps, depending upon 


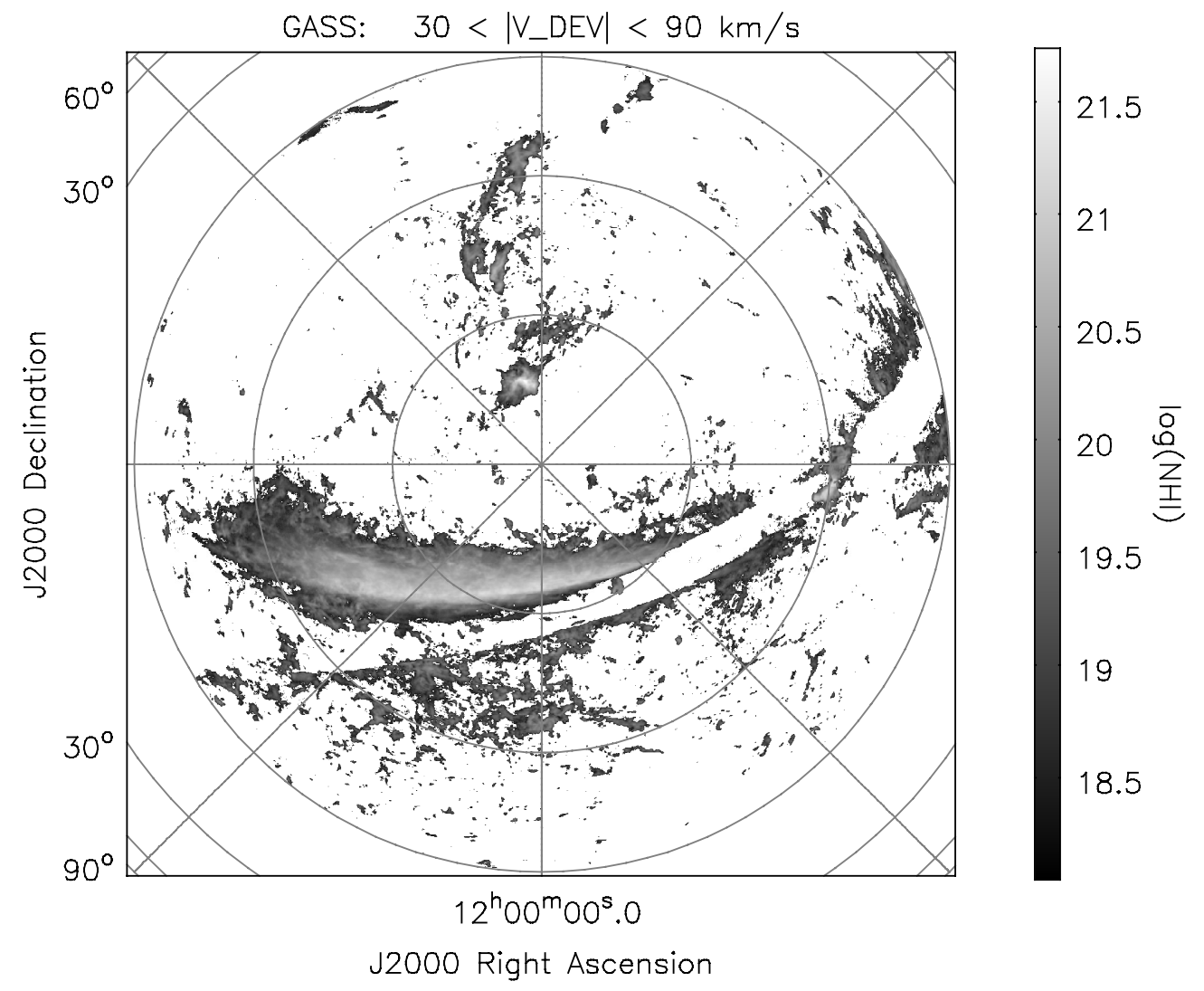

Figure 5.4: Column density map of intermediate velocity gas as seen with GASS $\left(30 \mathrm{~km} \mathrm{~s}^{-1} \leq\right.$ $\left|v_{\text {dev }}\right| \leq 90 \mathrm{~km} \mathrm{~s}^{-1}$ ) 


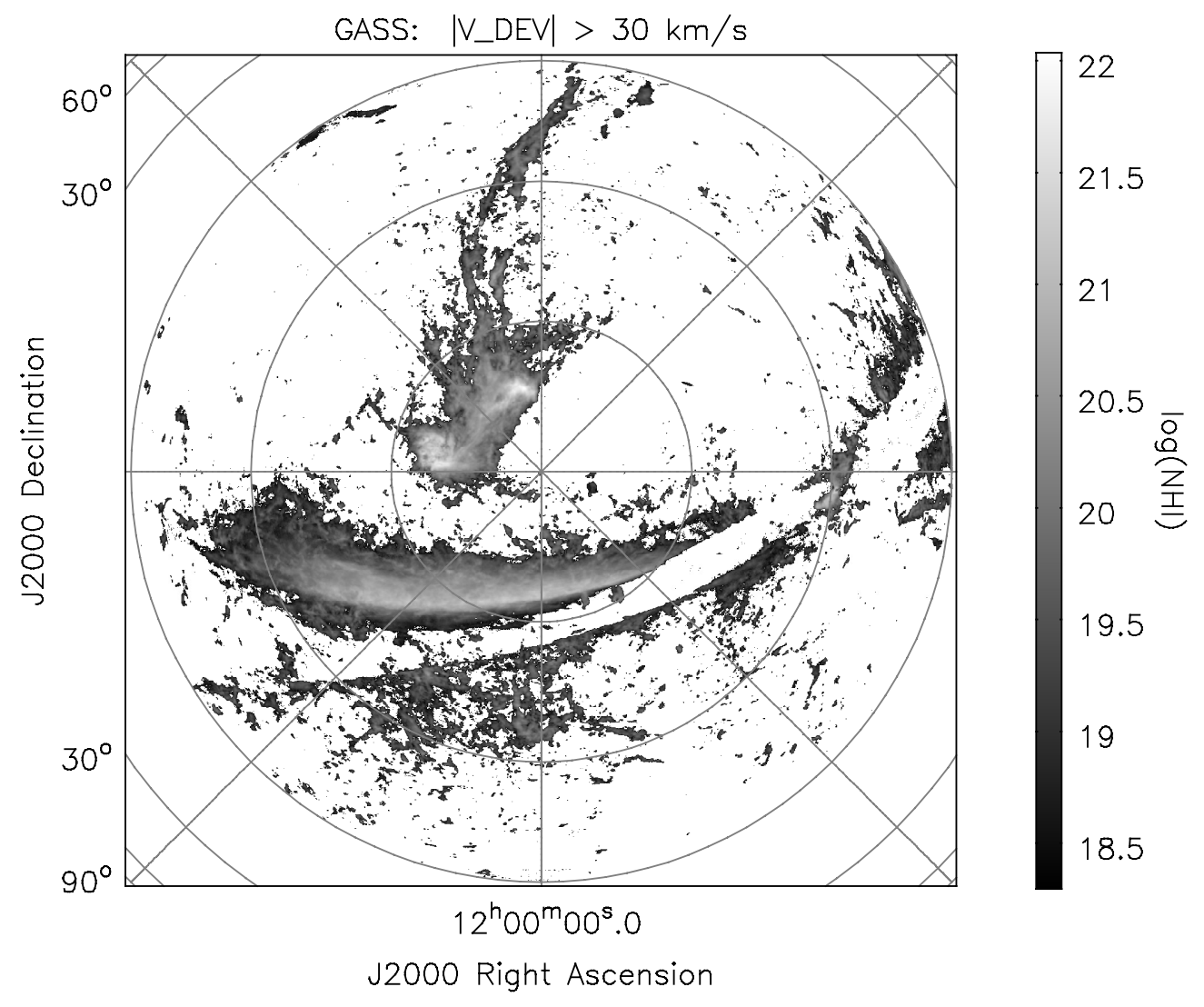

Figure 5.5: Column density map of both the high and intermediate velocity gas as seen with GASS $\left(30 \mathrm{~km} \mathrm{~s}^{-1} \leq\left|v_{d e v}\right|\right)$ 


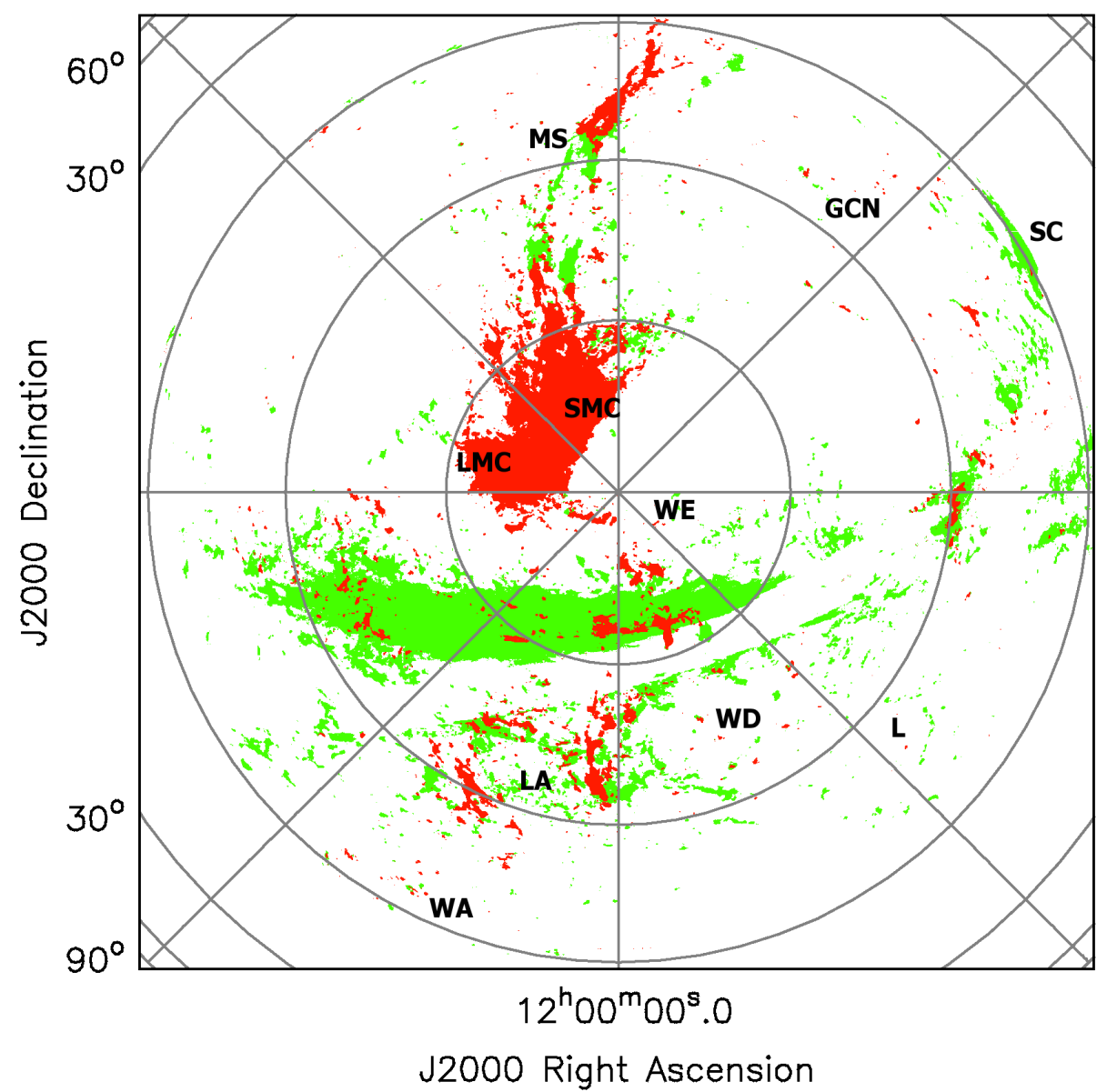

Figure 5.6: Two tone color map of the HVC and IVC gas. HVC material is in red, while the IVC is in green. Cloud Complexes are labeled. MS is the Magellanic Stream. SMC and LMC are the Large and Small Magellanic Cloud respectively. MS is the magellanic Stream. SC is Smith's Cloud. Other complex labels are taken from the literature (Wannier et al., 1972; Wakker \& van Woerden, 1991).

which difference was smaller. If $\left|v_{\mathrm{lsr}}-\mathrm{v}_{\min }\right|<\left|\mathrm{v}_{\mathrm{lsr}}-\mathrm{v}_{\max }\right|$, we took the difference between $v_{\mathrm{lsr}}$ and $\mathrm{v}_{\min }$. If $\left|\mathrm{v}_{\mathrm{lsr}}-\mathrm{v}_{\min }\right|>\left|\mathrm{v}_{\mathrm{lsr}}-\mathrm{v}_{\max }\right|$, we took the difference between $\mathrm{v}_{\mathrm{lsr}}$ and $\mathrm{v}_{\max }$.

Figure 5.7 shows the deviation velocity for the intermediate velocity gas, overlaid with the deviation velocity for the high velocity case. This is to show the contrasting velocities in regions where both populations overlap. 


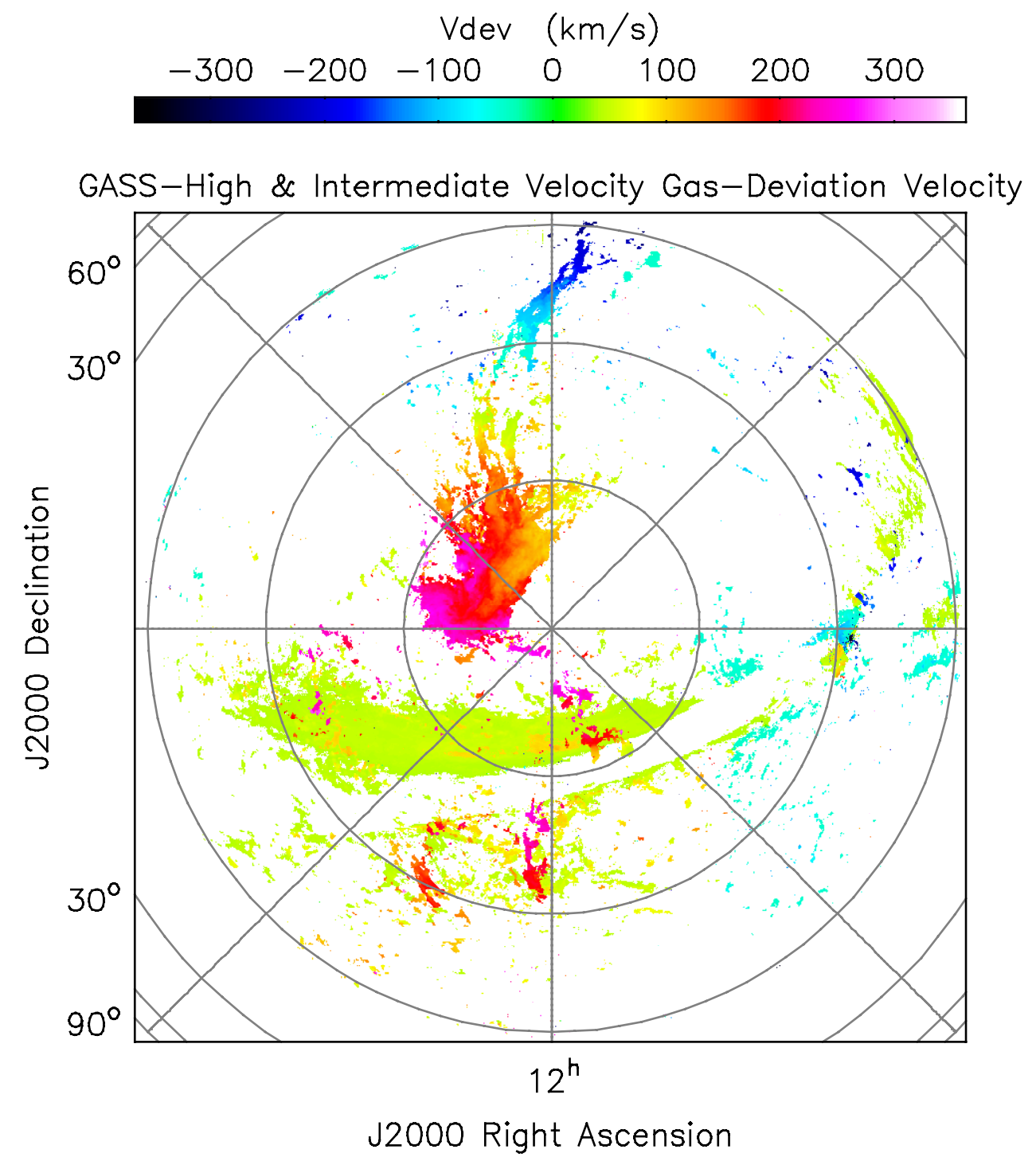

Figure 5.7: Deviation velocity map for both the high velocity and intermediate velocity gas. High velocity $\mathrm{H}$ I is overlaid on the intermediate velocity $\mathrm{H}$ I to highlight the contrasting velocities, especially in the Leading Arms of the Magellanic Stream. 


\subsection{Global Properties}

For this work, we consider the basic properties of the total sky covering fraction and column density distribution of the HVC and IVC gas. The column density distribution was calculated by summing up the emission over the entire GASS data set and converting to column density. Since the pixels in a ZEA projection have equal area, the column density distribution was calculated by determining the total number of pixels within a certain column density bin and dividing by the total number of spatial pixels in the GASS data, yielding a percentage.

The binning of the data is done as described in van Woerden et al. (2004) to provide a more reliable representation of the data. Specifically, data between $10^{18} \mathrm{~cm}^{-2}$ and $10^{19} \mathrm{~cm}^{-2}$ were binned by $10^{18} \mathrm{~cm}^{-2}$ intervals. Data between $10^{19} \mathrm{~cm}^{-2}$ and $10^{20} \mathrm{~cm}^{-2}$ were binned by $10^{19} \mathrm{~cm}^{-2}$ and scaled down by a factor of 10 . Data between $10^{20} \mathrm{~cm}^{-2}$ and $10^{21} \mathrm{~cm}^{-2}$ were binned by $10^{20} \mathrm{~cm}^{-2}$ and scaled down by 100 and so on. This is equivalent to binning by $10^{18} \mathrm{~cm}^{-2}$ over the whole data range, but also ensures that the high column density bins are not empty. The resulting distribution is similar to that of other surveys when they are properly scaled by the same method (Wakker, 2001; Hulsbosch \& Wakker, 1988). See Figure 5.8 for the results from the HVC case, IVC case, and when the HVC and IVC data are combined.

The distributions follow a power law with an index of $\alpha=-1.4 \pm 0.2$. This is similar to the index measured from Moss et al. (2013) for the case of HVCs (Moss et al., 2013). That work, however, focused on individual clouds, disregarding emission from the Galactic warp and flare. The result is slightly shallower when compared 


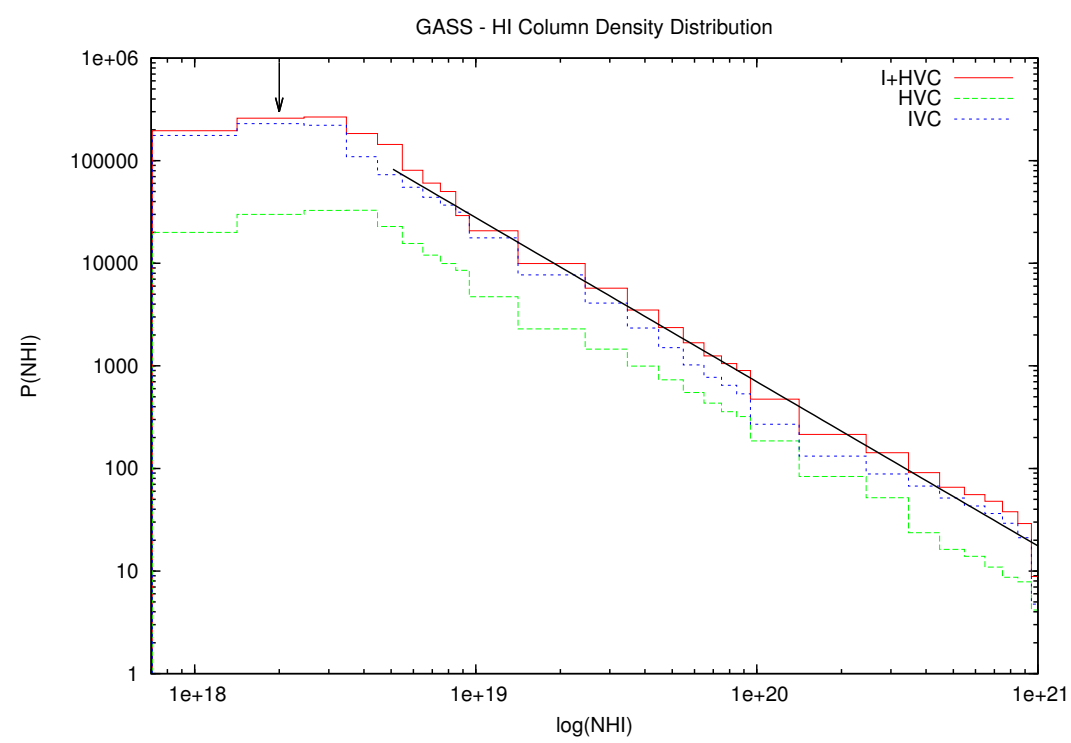

Figure 5.8: Column density distribution of the GASS survey for the HVC gas, IVC gas and when both populations are combined. The $\mathrm{x}$-axis is the column density in units of $\mathrm{cm}^{-2}$ and the $\mathrm{y}$-axis is the total percentage of sky coverage. The arrow indicates the sensitivity limit of the GASS survey. The black line is the power law fit for the I+HVC distribution $(\alpha=-1.4 \pm 0.2)$.

to the results of the HVC distribution from Putman et al. (2002), although they developed their distributions from the clouds as well, and not the overall emission. The sky covering fraction of the HVC gas down to $1.6 \times 10^{18} \mathrm{~cm}^{-2}$ is approximately 7\%. The IVC gas has a covering fraction of approximately $17 \%$ and both populations together give a total sky covering fraction of approximately $22 \%$. The result for the HVC case is lower than that reported by other surveys down to similar sensitivities (e.g. Hulsbosch \& Wakker, 1988; Murphy et al., 1995). This is likely due to the fact that for these surveys, the beam area is roughly twice as large $\left(\sim 30^{\prime}\right)$ as the one used for GASS and thus the emission from the previous surveys would appear to be more extended. A simple ratio of the beam sizes provides roughly a factor of four larger beam area. Assuming that small clouds become more extended, and the edges of the larger structures extend as well, would roughly scale the GASS results to the 
same general range as the previous surveys. The inclusion of the large, extended HVC Complexes of the northern sky which, aside from the Magellanic system, is lacking in the southern sky can also increase the measured sky covering fraction of anomalous velocity gas. These results are also lower than those reported in a sensitive Hi survey by Lockman et al. (2002) ( 37\%). That survey, however, defined high velocity emission as $\left|\mathrm{v}_{\mathrm{LSR}}\right| \geq 100 \mathrm{~km} \mathrm{~s}^{-1}$ and was more sensitive than GASS. An extrapolation of the power law fit of the GASS results to the same sensitivity gives a covering fraction of $\sim 33 \%$ for both the HVC and IVC emission, which is similar to the Lockman et al. (2002) result.

The turnover in the distributions occurs at the survey's sensitivity limit. This means one cannot determine an average or "most likely" column density from the anomalous velocity emission down to this limit. The sky covering fraction and column density distributions suggest that most of the anomalous velocity clouds in the southern sky have smaller angular scales with higher column densities than the clouds detected in the northern sky, which is evident upon inspection of the HVC/IVC maps. What emission is not Galactic or Magellanic appears to be in the form of small clouds.

Roughly $19 \%$ of the anomalous velocity emission in the southern sky is at negative LSR velocities. However, most of this effect can be attributed to the rotation of the Galaxy. Transforming to the Galactic Standard of Rest frame, GSR, which is relative to Milky Way rotation has been shown to eliminate most of the velocity bias. See Wakker (2001) and van Woerden et al. (2004) for a more detailed discussion of this. We can also calculate the deviation velocity distribution of the anomalous velocity gas (Figure 5.9). There is an asymmetry present in the distribution. The 
large peak around $\pm 30 \mathrm{~km} \mathrm{~s}^{-1}$ is due to the Galactic warp and flare present in the maps. The increased amount of emission between $120 \mathrm{~km} \mathrm{~s}^{-1}$ and $250 \mathrm{~km} \mathrm{~s}^{-1}$ is due to the Magellanic Clouds and parts of the Magellanic Stream. There is more positivedeviation velocity emission in the southern sky than negative, which is also apparent in previous surveys (e.g. see Wakker, 2004).

There has been no word of an IVC catalogue using the LAB survey as of 2011 (Witt et al., 2011). Even so, the images produced by Tobias Westmeier ${ }^{1}$ (Westmeier, 2007) resemble our results upon visual inspection. When Morras et al. (2000) undertook a closer inspection of the IAR component of the LAB survey (Bajaja et al., 2005), several features were revealed in the maps, many of them very similar to those in our map; specifically, the extended emission around the Leading Arms of the Magellanic Stream at positive Galactic latitudes. Nevertheless, our IVC maps are the most detailed and complete for the southern sky to date.

\subsection{Individual Features}

\subsubsection{High Velocity Emission}

The most obvious features from the HVC map are the Large and Small Magellanic clouds (LMC and SMC in Figure 5.6), as well as the Magellanic Stream (MS). Also visible are the many filaments and bifurcations extending off the Magellanic Clouds as discussed by Stanimirović et al. (2008), and the Leading Arm material (LA). Work by Venzmer et al. (2012) has investigated the Leading Arms of the

\footnotetext{
${ }^{1}$ http://www.atnf.csiro.au/people/Tobias.Westmeier/research_hvcsky.php
} 


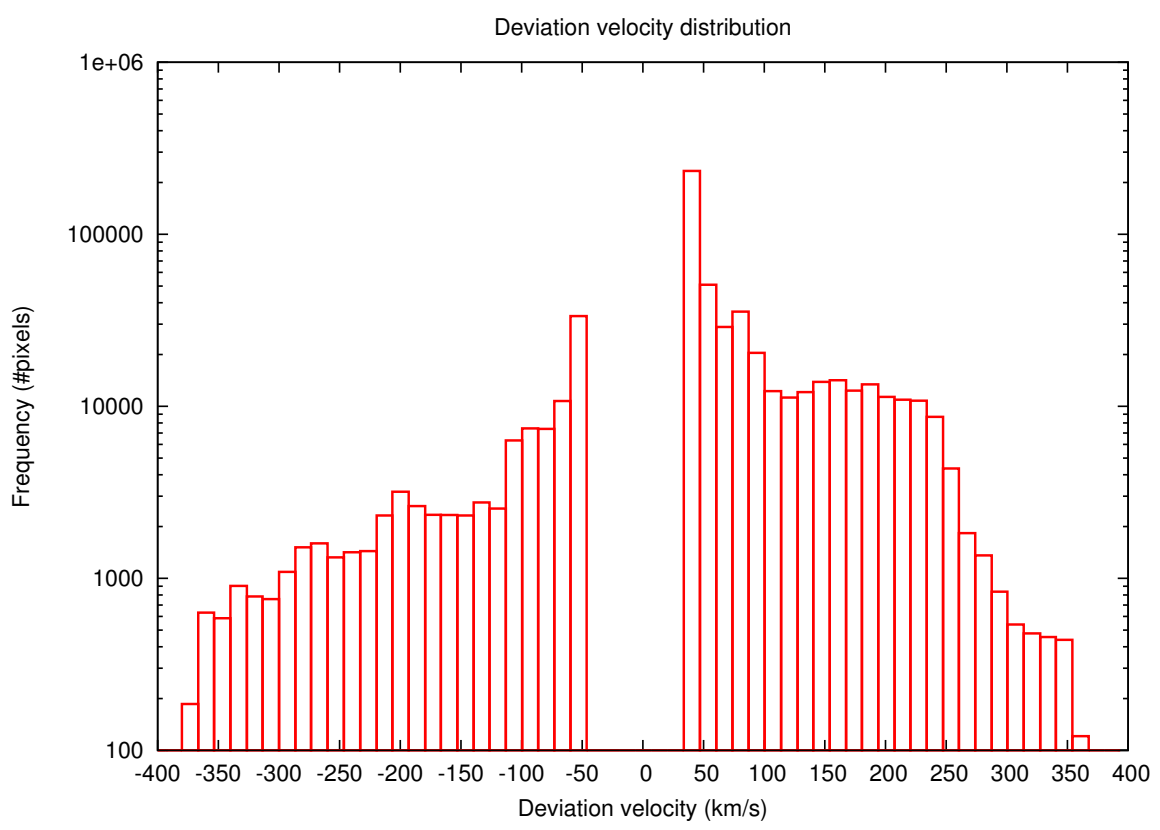

Figure 5.9: Deviation velocity distribution for both the HVC an IVC gas. The velocity is binned in increments of approximately $7 \mathrm{~km} \mathrm{~s}^{-1}$.

Magellanic Stream using GASS. Even though they filter Milky Way emission based on the LSR velocity, the structures of the Leading Arms they observe closely match those from this work. The velocity gradient along the MS is obvious upon inspection. There is also emission seen from the Complex GCN. Clouds also appear near the Galactic Center, which likely is due to the non-circular velocities of the gas located there. The HVC Complex WE can be seen to the right of the MCs. Some clouds from Complex WD are visible to the right of the Leading Arms at positive Galactic latitude. A small portion of Complex WA is visible at the bottom left. The rest of the emission is in the form of small clouds. A thorough comparison of the emission in the HVC maps to those of other surveys is ongoing. 


\subsubsection{Intermediate Velocity Emission}

The IVC map also contains parts of the MS and the SMC, including streams coming from both structures. Clouds from Complex GCN appear as well. The largest feature seen at low deviation velocity is due to the Galactic warp. Most emission from it lies very near the velocity cutoff as expected. There is a clear kinematic distinction between the Galactic plane material and Complex L, but there appears to be a kinematic connection between Complex L and the ejecta clouds detected in the GASS pilot region, which is likely ejected material due to supernovae or stellar winds (Ford et al., 2008). Emission can also be seen at higher Galactic latitudes (toward the bottom of the maps), including material spatially coincident with the Leading Arms seen in the HVC map. A closer inspection of the maps reveals a decreasing gradient in deviation velocity $\left(\sim 50 \mathrm{~km} \mathrm{~s}^{-1}\right)$ in the Leading Arms to higher latitudes and a slightly decreasing one $\left(\sim 15 \mathrm{~km} \mathrm{~s}^{-1}\right)$ in the surrounding IVC material. Evidence is emerging that some of the Leading Arm material is interacting with the Galactic disk (McClure-Griffiths et al., 2008; Venzmer et al., 2012). It may be possible that this IVC gas is Galactic material pulled up from the disk or stripped from the Leading Arms due to this interaction, but that remains uncertain.

The Smith Cloud (Smith, 1963) (SC in Figure 5.6) also appears at the extreme edge of the map. As shown in Chapter 4, there is similar morphology between the Smith Cloud and one of the M31-M33 clouds (Cloud 4). The Smith Cloud may very well represent a cloud in the late stages of accretion onto the Galaxy (Lockman et al., 
2008) and may represent the ultimate fate of the M31-M33 clouds. Comparison of the IVC features seen in the GASS data to those of other H i surveys is ongoing.

\subsection{Conclusions}

Our maps of the anomalous velocity southern sky are the most detailed to date. Specifically, the maps have both high spatial and velocity resolution, as well as good sensitivity. Some IVC emission near the warp may be due to an interaction between the Galactic plane and Leading Arms. Examining the deviation velocity distribution shows that asymmetry exists between $\mathrm{H}$ i at positive and negative deviation velocities, with the larger amount of the positive velocity gas being due to the Magellanic system and Galactic warp. Examining the column density distribution reveals that there is no clear peak seen in the column density distribution of the $\mathrm{H}$ i emission down to the

survey sensitivity. It appears that, apart from the Magellanic system, most of the HVC and IVC emission in the southern sky in is the form of small clouds, in contrast to the large cloud complexes seen in the north.

There is a kinematical connection between Complex L and $\mathrm{H}$ I ejecta clouds, suggesting that complex L may be the result of gas removed from the disk by supernovae. The Smith Cloud may be a good representation of the final fate of the H I clouds now known to exist between the Local Group galaxies M31 and M33 and there may be similar clouds in the GASS data. A detailed comparison of the features seen in GASS to those of other surveys is ongoing. 
We can also use these data to study the two-phase temperature structure seen in some of the HVC/IVC gas, which can help us better understand the surrounding halo environment and the fate of the anomalous velocity gas (e.g. Wolfire et al., 1995). A thorough understanding of the HVC/IVC phenomenon is needed to search for analogies or any clues as to the origins and possible fate of the M31-M33 H I Clouds (Wolfe et al., 2013). Developing methods for this with clouds around the Milky Way will be important when we can obtain higher resolution data of the Local Group clouds. 


\section{Chapter 6}

\section{Conclusions and Future Work}

\subsection{The M31-M33 H i clouds}

A decade ago, Braun \& Thilker (2004) discovered a faint stream of $\mathrm{H}$ I between M31 and M33 and concluded that they were seeing a condensing intergalactic filament. While a better understanding of the stellar components between M31 and M33 has developed since then (e.g McConnachie et al., 2009), our knowledge of the gas was still lacking.

We have shown that the GBT is more than capable, with careful planning, to map extremely diffuse H I. Although we did discover a problem with the GBT Spectrometer, it was soon remedied, still making the GBT the only single dish telescope that can do this work reliably, thanks in large part to its design. The new GBT spectrometer, VEGAS, makes this even better, given its high dynamic range.

We have mapped part of the M31-M33 stream in detail, and find H I clouds a few kiloparsecs in size, similar to dwarfs but really unlike any Local Group object. All of the H i mass discovered by the Westerbork survey that overlaps our survey appears to reside in these clouds. The kinematics and lack of stars suggest that they are likely the result of a condensing filament, but we still cannot rule out a tidal feature. In order to do so, we need to map more areas around M31, and potentially M33, to better understand how the emission really is distributed, both in space and velocity. 
Pointings to the northwest of M31 indicate that the faint emission seen there is likely due to foreground Hi from the Milky Way and/or part of M31's HVC population. Again, mapping of this area is also needed. GBT observations of the Local Group are ongoing and will continue into the future.

We will also need higher resolution data, both spatially and in velocity. These clouds may very well resolve into smaller structures at higher column densities if we observe them at higher spatial resolution. Higher velocity resolution will also allow us to truly probe for line broadening and search for two-phase temperature structure in these clouds. That information can potentially tell us something about the environment surrounding the clouds. The VLA, in D configuration, would take on the order of 60 hours to map the brightest clouds. We do plan on re-reducing all of our data at higher velocity resolution for a thorough study of the cloud kinematics.

Absorption line measurements will also prove quite informative. There are a few bright sight lines near the M31-M33 clouds. HST observations can reveal information about the environment surrounding them, such as the ionization, and potentially provide us with a measurement of the metallicities. Low metallicity gas would suggest a primordial origin, furthering the possibility of a filament, while metallicities similar to M31, M33 or some other object would further support a tidal explanation for the Local Group clouds. If our ongoing $\mathrm{H}$ i observations of the region can provide detections along suitable sight lines, then HST observations will be pursued. 


\subsection{HVCs and IVCs with GASS}

We have also examined the H i that exists around our own Galaxy, in the form of HVCs and IVCs, by specifically using GASS to produce all sky maps of the HVC and IVC emission. We have filtered out Milky Way emission from the GASS data by employing the deviation velocity as opposed to using $\mathrm{v}_{\mathrm{LSR}}$. These maps are the most detailed of anomalous velocity emission to date, particularly for IVCs in the southern sky. The maps clearly show features that represent different evolutionary paths for the H I, such as tidal features (e.g. the Magellanic System), accretion in the case of Smith's Cloud and Galactic fountain material. It is possible that some of the material seen in GASS may represent the end result of the M31-M33 clouds evolutionary path. Thus, studying how H a accretes onto galaxies like our Milky Way is important, if we wish to understand how galaxies can continue to acquire fresh gas to form new stars.

Our analysis of the global properties leads us to conclude that most of the anomalous velocity clouds in the southern sky are typically smaller than the clouds and cloud complexes in the north. This is in agreement with the recent work by (Moss et al., 2013), who focused their analysis of the GASS data on the actual cloud properties and not the overall, global emission.

Because of the excellent velocity resolution of the GASS data, it is our plan to begin fitting and mapping the two phase temperature structure of the $\mathrm{H}$ a across the southern sky. This is important, because if we can determine whether or not an H I cloud has two component structure, it will then help us to learn some of the most basic properties of Galactic halo, such as the temperature and pressure (e.g. Wolfire 
et al., 1995). Given the wealth of information contained in the GASS data, there is no doubt that more interesting discoveries are waiting to be made with it. In the end, we wish to search for any and all analogous properties of the HVCs and IVCs around our own Galaxy to those of the M31-M33 clouds. 


\section{Appendix A}

\section{The H I Hyperfine Transition}

The neutral hydrogen atom, or $\mathrm{H} \mathrm{I}$, contains a single electron in an orbital around a single proton. Each particle's angular momentum includes both an orbital term $\overrightarrow{\mathbf{L}}$ and a spin term $\overrightarrow{\mathbf{S}}$ such that the total angular momentum becomes $\overrightarrow{\mathbf{F}}=\overrightarrow{\mathbf{L}}+\overrightarrow{\mathrm{S}}$. The orbital angular momentum term for both particles is zero for the $\mathrm{H}_{\mathrm{I}}$ ground state $(\overrightarrow{\mathbf{L}}=0)$. The proton and electron are spin $\frac{1}{2}$ particles, so their spins can be either "up" or "down". This means that the z-component of their spin angular momentum $\mathrm{S}_{z}$ can be $\pm \frac{\hbar}{2}$. Thus, there are four possible states for $\mathrm{H} \mathrm{I}$, with a total spin magnitude of either $\mathrm{F}=0$ or $\mathrm{F}=1$.

The difference in energy between these states $\Delta \mathrm{E}_{\mathrm{pot}}$ is due to the interaction of the magnetic field inside of the proton $\overrightarrow{\mathbf{B}_{\mathbf{p}}}$ with the magnetic moment of the electron $\overrightarrow{\mu_{\mathrm{e}}}$ (e.g. Griffiths, 1999). Specifically, we have

$$
\mathrm{E}_{\mathrm{pot}}=-\mathrm{P}\left(\overrightarrow{\mu_{\mathrm{e}}} \cdot \overrightarrow{\mathbf{B}_{\mathrm{p}}}\right)
$$

where $P$ is the probability the electron is found within the radius of the proton, $R_{p}$. To calculate P, we use the ground state H I wave function (Shankar, 1994)

$$
\left|\psi_{0}\right\rangle \Longleftrightarrow \frac{1}{\sqrt{\pi \mathrm{a}_{\mathrm{o}}^{3}}} \mathrm{e}^{-\frac{\mathrm{r}}{\mathrm{a}_{\mathrm{o}}}}
$$


where $\mathrm{a}_{\mathrm{o}}$ is the Bohr radius. Thus, we have

$$
\mathrm{P}=\int_{0}^{2 \pi} \mathrm{d} \phi \int_{0}^{\pi} \sin \theta \mathrm{d} \theta \int_{0}^{\mathrm{R}_{\mathrm{p}}}\left|\psi_{0}\right|^{2} \mathrm{r}^{2} \mathrm{dr} .
$$

Integrating and Taylor expanding in powers of $\frac{2 \mathrm{R}_{\mathrm{p}}}{\mathrm{a}_{\mathrm{o}}}$ gives

$$
\mathrm{P}=\frac{4 \mathrm{R}_{\mathrm{p}}^{3}}{3 \mathrm{a}_{\mathrm{o}}^{3}} \quad\left(\mathrm{R}_{\mathrm{p}} \ll \mathrm{a}_{\mathrm{o}}\right) .
$$

The magnetic moment of a particle with charge $q$ and mass $m$ can be defined in terms of its spin (Shankar, 1994)

$$
\vec{\mu} \equiv \mathrm{g} \frac{\mathrm{q}}{2 \mathrm{~m}} \overrightarrow{\mathrm{S}}
$$

where $\mathrm{g}$ is the spin g-factor. Inside the proton, the magnetic field is assumed to be constant and can be written classically as (Griffiths, 1999)

$$
\overrightarrow{\mathbf{B}_{\mathbf{p}}}=\frac{\mu_{0}}{2 \pi \mathrm{R}_{\mathrm{p}}^{3}} \overrightarrow{\mu_{\mathbf{p}}}
$$

Inserting Eq. A.6 into A.1 and applying the definitions of the magnetic moment of the electron and proton gives

$$
E_{\text {pot }}=g_{e} g_{p} \frac{\mu_{0} e^{2}}{6 \pi a_{o}^{3} m_{e} m_{p}} \overrightarrow{\mathbf{S}_{e}} \cdot \overrightarrow{\mathbf{S}_{p}}
$$


To determine $\overrightarrow{\mathbf{S}_{\mathbf{e}}} \cdot \overrightarrow{\mathbf{S}_{\mathbf{p}}}$, we first take the scalar product of $\overrightarrow{\mathbf{F}}$ with itself and rearrange, giving

$$
\overrightarrow{\mathrm{S}_{\mathrm{e}}} \cdot \overrightarrow{\mathrm{S}_{\mathrm{p}}}=\frac{1}{2}\left(\overrightarrow{\mathbf{F}} \cdot \overrightarrow{\mathbf{F}}-\overrightarrow{\mathrm{S}_{\mathrm{e}}} \cdot \overrightarrow{\mathrm{S}_{\mathrm{e}}}-\overrightarrow{\mathrm{S}_{\mathrm{p}}} \cdot \overrightarrow{\mathrm{S}_{\mathrm{p}}}\right)
$$

and thus

$$
\overrightarrow{\mathrm{S}_{\mathbf{e}}} \cdot \overrightarrow{\mathrm{S}_{\mathbf{p}}}|\psi\rangle=\frac{\hbar^{2}}{2}\left[\mathrm{~F}(\mathrm{~F}+1)-\mathrm{S}_{\mathrm{e}}\left(\mathrm{S}_{\mathrm{e}}+1\right)-\mathrm{S}_{\mathrm{p}}\left(\mathrm{S}_{\mathrm{p}}+1\right)\right]|\psi\rangle
$$

$\mathrm{S}_{\mathrm{e}}$ and $\mathrm{S}_{\mathrm{p}}$ are both $\frac{1}{2}$. So, for the $\mathrm{F}=0$ and $\mathrm{F}=1$ state we have, respectively

$$
\begin{gathered}
\overrightarrow{\mathrm{S}_{\mathbf{e}}} \cdot \overrightarrow{\mathrm{S}_{\mathbf{p}}}|\psi\rangle_{\mathrm{F}=0}=-\frac{3}{4} \hbar^{2}|\psi\rangle \\
\overrightarrow{\mathrm{S}_{\mathbf{e}}} \cdot \overrightarrow{\mathrm{S}_{\mathbf{p}}}|\psi\rangle_{\mathrm{F}=1}=\frac{1}{4} \hbar^{2}|\psi\rangle .
\end{gathered}
$$

Applying Eq. A.10 and A.11 to A.7 and taking the difference between these two states gives, plugging in known values for the constants

$$
\Delta \mathrm{E}_{\text {pot }}=g_{\mathrm{e}} \mathrm{g}_{\mathrm{p}} \frac{\mu_{0} \mathrm{e}^{2} \hbar^{2}}{6 \pi \mathrm{a}_{\mathrm{o}}^{3} \mathrm{~m}_{\mathrm{e}} \mathrm{m}_{\mathrm{p}}}=9.43 \times 10^{-25} \mathrm{~J} .
$$

This is the energy released, in the form of a photon, when the total spin decays, or "flips" from the higher $\mathrm{F}=1$ state to the lower $\mathrm{F}=0$ state. This corresponds to a frequency of

$$
\nu=\frac{\Delta \mathrm{E}_{\mathrm{pot}}}{h}=1420.406 \mathrm{MHz}
$$


or a wavelength of

$$
\lambda=\frac{c}{\nu}=21.106 \mathrm{~cm}
$$

The temperature associated with this transition is

$$
\mathrm{T} \sim \frac{h \nu}{k}=0.0683 \mathrm{~K}
$$

which is less than the cosmic microwave background (Fixsen, 2009). Thus, if hydrogen gas exists in the neutral state, then $21 \mathrm{~cm}$ transitions will take place.

To estimate the emission rate, we use the Einstein $A$ coefficient $A_{10}$ for a magnetic dipole. Specifically, we have (e.g. Draine, 2011)

$$
\mathrm{A}_{10}=\frac{<\mathrm{P}_{10}>}{h \nu_{10}}
$$

$<\mathrm{P}>$ is the average power radiated by the dipole (e.g Griffiths, 1999)

$$
<\mathrm{P}>=\frac{4 \mu_{0} \pi^{3}}{3 \mathrm{c}^{3}} \nu^{4}|\mu|^{2}
$$

where $\mu$ is the magnetic moment of the dipole. In this case, $\left|\mu_{10}\right|=\frac{\hbar \mathrm{e}}{2 \mathrm{~m}_{\mathrm{e}}}$ is the Bohr magneton. Thus we have

$$
\mathrm{A}_{10}=\frac{4 \mu_{0} \pi^{3}}{3 h \mathrm{c}^{3}} \nu^{3}\left|\mu_{10}\right|^{2} \approx 2.85 \times 10^{-15} \mathrm{~s}^{-1}
$$


The average radiative half life of the $21 \mathrm{~cm}$ line then is

$$
\tau_{1 / 2}=\mathrm{A}_{10}^{-1} \approx 3.5 \times 10^{14} \mathrm{~s} \approx 11.1 \text { million years. }
$$




\section{Appendix B}

\section{Radiative Transfer and the $\mathrm{H}_{\mathrm{I}}$ Line}

Imagine radiation of specific intensity $\mathrm{I}_{\nu}$ incident on a parcel of gas with incremental path length ds. A fraction of this radiation will be absorbed by the gas, $\mathrm{dI}_{\nu-}$, while emission from the gas will also add to the intensity by some amount $\mathrm{dI}_{\nu+}$. Thus the total change in the intensity of the radiation along the parcel of gas will be

$$
\mathrm{dI}_{\nu}=\mathrm{dI}_{\nu+}-\mathrm{dI}_{\nu-} .
$$

It is typical to define the amount of radiation absorbed and emitted as

$$
\begin{gathered}
\mathrm{dI}_{\nu-}=-\kappa_{\nu} \mathrm{I}_{\nu} \mathrm{ds} \\
\mathrm{dI}_{\nu+}=\epsilon_{\nu} \mathrm{ds},
\end{gathered}
$$

respectively, where $\kappa_{\nu}$ is the absorption coefficient and $\epsilon_{\nu}$ is the emissivity. Equations B.2 and B.3, along with many of the following formulas and definitions can be found in Draine (2011). Thus equation B.1 becomes

$$
\frac{\mathrm{dI}_{\nu}}{\mathrm{ds}}=\epsilon_{\nu}-\kappa_{\nu} \mathrm{I}_{\nu},
$$


which is the equation of radiative transfer. In local thermodynamic equilibrium, Kirchhoff's Law states that

$$
\frac{\epsilon_{\nu}}{\kappa_{\nu}}=\mathrm{B}_{\nu}(\mathrm{T})
$$

Here, $\mathrm{B}_{\nu}(\mathrm{T})$ is the Planck function with $\mathrm{T}$ being the (kinetic) temperature of the gas

$$
\mathrm{B}_{\nu}(\mathrm{T})=\frac{2 h \nu^{3}}{c^{2}} \frac{1}{\mathrm{e}^{h \nu / k T}-1} .
$$

The optical depth, $\tau_{\nu}$, is defined such that

$$
\mathrm{d} \tau_{\nu}=-\kappa_{\nu} \mathrm{ds}
$$

So, substituting Eq. B.5 and B.7 into B.4 gives

$$
\frac{\mathrm{dI}_{\nu}}{\mathrm{d} \tau_{\nu}}=\mathrm{I}_{\nu}-\mathrm{B}_{\nu}(\mathrm{T})
$$

The solution to Eq. B.8 is

$$
\mathrm{I}_{\nu}(\mathrm{s})=\mathrm{I}_{\nu}(0) \mathrm{e}^{-\tau_{\nu}(\mathrm{s})}+\int_{0}^{\tau_{\nu}(\mathrm{s})} \mathrm{B}_{\nu}(\mathrm{T}) \mathrm{e}^{-\tau_{\nu}(\mathrm{s})} \mathrm{d} \tau_{\nu}(\mathrm{s}) .
$$

One can expand the exponential of Eq. B.6 for the case of $(h \nu / k T \ll 1)$ which results in the Rayleigh-Jeans law

$$
B_{\nu}(\mathrm{T})=\frac{2 k}{c^{2}} \nu^{2} \mathrm{~T},
$$


which is valid for most thermal radio sources. From this, one can define the brightness temperature $\mathrm{T}_{\mathrm{B}}$ of a thermal or non-thermal source such that

$$
\mathrm{I}_{\nu}=\frac{2 k}{\mathrm{c}^{2}} \nu^{2} \mathrm{~T}_{\mathrm{B}}
$$

Thus, $\mathrm{T}_{\mathrm{B}}$ is the the temperature a non-thermal source would need to produce blackbody emission at the observed intensity $\mathrm{I}_{\nu}$. Substituting Eq. B.10 and B.11 into B.9 gives

$$
\mathrm{T}_{\mathrm{B}}(\mathrm{s})=\mathrm{T}_{\mathrm{B}}(0) \mathrm{e}^{-\tau_{\nu}(\mathrm{s})}+\int_{0}^{\tau_{\nu}(\mathrm{s})} \mathrm{Te}^{-\tau_{\nu}(\mathrm{s})} \mathrm{d} \tau_{\nu}(\mathrm{s})
$$

If the gas is assumed to be isothermal, where $\mathrm{T}$ is constant, then Eq. B.12 can be directly integrated

$$
\mathrm{T}_{\mathrm{B}}(\mathrm{s})=\mathrm{T}_{\mathrm{B}}(0) \mathrm{e}^{-\tau_{\nu}(\mathrm{s})}+\mathrm{T}\left(1-\mathrm{e}^{-\tau_{\nu}(\mathrm{s})}\right)
$$

If $\tau \gg 1$, then the gas is said to be optically thick and we have

$$
\mathrm{T}_{\mathrm{B}} \approx \mathrm{T}
$$

If $\tau \ll 1$, then the gas is optically thin and we have

$$
\mathrm{T}_{\mathrm{B}} \approx \tau_{\nu} \mathrm{T}
$$

It can be shown (Draine, 2011) that the emissivity for the H I line can be written as 


$$
\epsilon_{\nu} \approx \frac{3}{16 \pi} A_{10} h \nu_{10} \phi(\nu) n(\mathrm{HI})
$$

where $\mathrm{A}_{10}$ is the Einstein A coefficient and $\nu_{10}$ is the $21 \mathrm{~cm}$ transition frequency. See Appendix A. $\mathrm{n}(\mathrm{HI})$ is the number density of $\mathrm{H}$ I atoms and $\phi(\nu)$ is the normalized line profile determined by the velocity distribution of the $\mathrm{H}$.. If we assume the gas is optically thin, where absorption is ignored, then we can solve the radiative transfer equation and integrate over $\nu$ to give

$$
\int\left(\mathrm{I}_{\nu}-\mathrm{I}_{\nu}(0)\right) \mathrm{d} \nu=\iint \epsilon_{\nu} \mathrm{ds} \mathrm{d} \nu=\frac{3}{16 \pi} \mathrm{A}_{10} h \nu_{10} \mathrm{~N}(\mathrm{HI})
$$

where $\mathrm{N}\left(\mathrm{HI}_{\mathrm{I}}\right)$ is the $\mathrm{H}$ I column density. It is typical to define the radial velocity of the gas v, along the line of sight, using the Doppler effect such that

$$
\mathrm{dv}=\mathrm{c} \frac{\mathrm{d} \nu}{\nu_{10}}
$$

Substituting this into B.17 and converting $\mathrm{I}_{\nu}$ to brightness temperature gives

$$
\int\left(\mathrm{T}_{\mathrm{B}}-\mathrm{T}_{\mathrm{B}}(0)\right) \mathrm{dv}=\frac{3}{32 \pi} \mathrm{A}_{10} \frac{\mathrm{hc}^{3}}{2 k \nu_{10}^{2}} \mathrm{~N}(\mathrm{H} \mathrm{I})
$$

which is the area under the spectral line in velocity. This will be defined as

$$
\int\left(\mathrm{T}_{\mathrm{B}}-\mathrm{T}_{\mathrm{B}}(0)\right) \mathrm{dv} \equiv \int_{\text {line }} \mathrm{T}_{\mathrm{B}} \mathrm{dv}
$$


Rearranging and plugging in the known values for the constants, we have

$$
\frac{\mathrm{N}(\mathrm{H} \mathrm{I})}{\mathrm{cm}^{-2}}=1.822 \times 10^{18} \int_{\text {line }}\left(\frac{\mathrm{T}_{\mathrm{B}}(\mathrm{v})}{\mathrm{K}}\right)\left(\frac{\mathrm{dv}}{\mathrm{km} \mathrm{s}^{-1}}\right) .
$$

If we assume that the gas follows a Maxwellian distribution in velocity due to thermal motions, then one can use the line width, or full width at half maximum (FWHM) of the line to estimate the kinetic temperature $\mathrm{T}_{\mathrm{K}}$

$$
\phi(v) \sim e^{-\frac{m_{H I}}{2 k T} v^{2}} \sim e^{-\frac{4 l n(2)}{F W H M^{2}} v^{2}} .
$$

Thus we have that

$$
\mathrm{T}_{\mathrm{K}} \sim \frac{\mathrm{m}_{\mathrm{HI}}}{8 \ln (2) \mathrm{k}}(\mathrm{FWHM})^{2} .
$$

For this written work, typical line widths are on the order of $25 \mathrm{~km} \mathrm{~s}^{-1}$ (Wolfe et al., 2013), which gives $\mathrm{T} \sim 10^{4} \mathrm{~K}$. Typical brightness temperatures are only a few dozen or so mK. Thus we have that $\tau \ll 1$.

One can also take the integrated intensity of the emission and calculate the total $\mathrm{H}$ i mass over a telescope beam. The beam projects an area at some distance $\mathrm{D}$ over a solid angle $\Omega$ such that the covered area is $\mathrm{A}=\mathrm{D}^{2} \Omega$. The total number of $\mathrm{H}$ I atoms $\mathrm{N}_{\text {tot }}$ is simply the column density times the area, so the total H I mass over the telescope beam then becomes

$$
\mathrm{M}_{\mathrm{HI} \text {,beam }}=\mathrm{m}_{\mathrm{HI}} \mathrm{N}_{\text {tot }}=\mathrm{m}_{\mathrm{HI}} \mathrm{N}(\mathrm{HI}) \mathrm{A}=\mathrm{m}_{\mathrm{HI}} \mathrm{N}(\mathrm{HI}) \mathrm{D}^{2} \text {, }
$$


where $\mathrm{m}_{\mathrm{HI}}$ is the mass of the $\mathrm{H}$ I atom. Thus, to calculate the $\mathrm{H}$ I mass, one must have some knowledge of the distance to the object in question. Using Equation B.17 for $\mathrm{N}(\mathrm{HI})$, replacing $\mathrm{I}_{\nu}$ with the flux density $\mathrm{S}_{\nu}$ over one telescope beam, plugging in known values for the constants and rearranging, we have

$$
\left(\frac{\mathrm{M}_{\mathrm{HI}, \text { beam }}}{\mathrm{M}_{\odot}}\right)=2.36 \times 10^{5}\left(\frac{\mathrm{D}}{\mathrm{Mpc}}\right)^{2} \int_{\text {line }}\left(\frac{\mathrm{S}_{\nu}}{\mathrm{Jy}_{\mathrm{y}}}\right)\left(\frac{\mathrm{dv}}{\mathrm{km} \mathrm{s}^{-1}}\right)
$$

$\mathrm{M}_{\odot}$ is the solar mass. Jy is the Jansky unit of flux density, defined such that $1 \mathrm{Jy} \equiv 10^{-26} \frac{\mathrm{W}}{\mathrm{m}^{2} \mathrm{~Hz} \mathrm{sr}}$. 


\section{Appendix C}

\section{Radio Telescope and Spectral Line Fundamentals}

The power per unit spectral frequency $\mathrm{P}_{\nu}$ received by a radio telescope, in units of $\mathrm{W} \mathrm{Hz}^{-1}$, is defined such that

$$
\mathrm{P}_{\nu} \equiv \mathrm{A}_{\mathrm{e}} \cdot \frac{\mathrm{S}_{\nu}}{2}
$$

where $A_{e}$ is the effective collecting area, in $\mathrm{m}^{2}$, and $\mathrm{S}_{\nu}$ is the flux density of the radiation incident on the telescope in units of $\mathrm{W} \mathrm{m}^{-2} \mathrm{~Hz}^{-1}$. The factor of two arises when the radiation is unpolarized. Specifically, both orthogonal components will vary rapidly but will have the same power when averaged over long periods of time. In general, the power $\mathrm{dP}_{\nu}$ measured due to some flux density $\mathrm{dS}_{\nu}$ along a given sightline $(\theta, \phi)$ is defined such that $\mathrm{dP}_{\nu}=\frac{1}{2} \mathrm{~A}_{\mathrm{e}}(\theta, \phi) \mathrm{dS}_{\nu}$. Virtually all of the definitions and formulas used in this Appendix can be found in Stanimirovic et al. (2002) or at the national radio astronomy observatory's online course ${ }^{1}$.

If we were to place an antenna in a cavity at thermodynamic equilibrium, then we can use the Rayleigh-Jeans approximation for blackbody radiation and find that

$$
\mathrm{P}_{\nu}=\int \mathrm{A}_{\mathrm{e}}(\theta, \phi) \frac{\mathrm{d \textrm {S } _ { \nu }}}{2}=\int_{4 \pi} \mathrm{A}_{\mathrm{e}}(\theta, \phi) \frac{\mathrm{B}_{\nu}}{2} \mathrm{~d} \Omega=\frac{\mathrm{kT}}{\lambda^{2}} \int_{4 \pi} \mathrm{A}_{\mathrm{e}}(\theta, \phi) \mathrm{d} \Omega .
$$

\footnotetext{
${ }^{1}$ http://www.cv.nrao.edu/course/astr534/ERA.shtml
} 
Antenna temperature $T_{A}$ is defined as the power received per unit spectral frequency

$$
\mathrm{T}_{\mathrm{A}} \equiv \frac{\mathrm{P}_{\nu}}{\mathrm{k}}
$$

which is the temperature a resistor would be if it were radiating the same amount of power. Thus, we have, using Equations C.2 and C.3

$$
\int_{4 \pi} \mathrm{A}_{\mathrm{e}}(\theta, \phi) \mathrm{d} \Omega=\lambda^{2}
$$

The power gain of a transmitting antenna $\mathrm{G}(\theta, \phi)$ is defined as the ratio of the power transmitted per unit solid angle in some direction to that of an isotropic antenna transmitting at the same power. The normalized power pattern $\mathrm{P}_{\mathrm{n}}(\theta, \phi)$ is defined as

$$
\mathrm{P}_{\mathrm{n}}(\theta, \phi)=\frac{\mathrm{G}(\theta, \phi)}{\mathrm{G}_{\max }}
$$

A transmitter projects this pattern on the sky, generally consisting of a central main lobe, or main beam, and smaller side lobes extending outwards in solid angle. The beam solid angle is defined to be the power pattern integrated over all solid angles

$$
\Omega_{\mathrm{A}} \equiv \int_{4 \pi} \mathrm{P}_{\mathrm{n}}(\theta, \phi) \mathrm{d} \Omega=\frac{1}{\mathrm{G}_{\max }} \int_{4 \pi} \mathrm{Gd} \Omega .
$$

For an ideal lossless antenna, $\int_{4 \pi} \mathrm{Gd} \Omega=4 \pi$ and thus we have that

$$
\Omega_{\mathrm{A}}=\frac{4 \pi}{\mathrm{G}_{\max }} .
$$


The reciprocity theorem states that since Maxwell's equations are time reversible, a transmitting antenna with gain $\mathrm{G}$ is equivalent to a receiving antenna with effective area $A_{e}$. Specifically, we have that

$$
\mathrm{A}_{\mathrm{e}}(\theta, \phi)=\frac{\lambda^{2} \mathrm{G}(\theta, \phi)}{4 \pi}
$$

If we assume that radiation of some brightness temperature $\mathrm{T}_{\mathrm{B}}$ subtends some solid angle of the telescope beam $\Omega_{\mathrm{S}}$, then we have that

$$
\mathrm{kT}_{\mathrm{A}}=\mathrm{P}_{\nu} \approx \frac{\mathrm{kT}}{\lambda^{2}} \frac{\lambda^{2} \mathrm{G}_{\max }}{4 \pi} \Omega_{\mathrm{S}}=\mathrm{kT}_{\mathrm{B}} \frac{\Omega_{\mathrm{S}}}{\Omega_{\mathrm{A}}},
$$

See Appendix B for a discussion of brightness temperature. So, we have that

$$
\mathrm{T}_{\mathrm{A}} \approx \mathrm{T}_{\mathrm{B}} \frac{\Omega_{\mathrm{S}}}{\Omega_{\mathrm{A}}}
$$

If our source is large, meaning that it extends beyond the telescope beam, then $\Omega_{\mathrm{S}}=\Omega_{\mathrm{A}}$ and the antenna temperature and brightness temperature are the same. So, $T_{B}$ is the unit typically used to quantify the intensity of extended sources. The aperture efficiency is also defined as the ratio of the effective area to the geometric area $\mathrm{A}_{\text {geom }}$.

$$
\eta_{\mathrm{a}} \equiv \frac{\mathrm{A}_{\mathrm{e}}}{\mathrm{A}_{\text {geom }}}
$$

The power received by a radio telescope is typically bounced off of a primary reflector onto a secondary sub reflector or directly into the receiver assembly, or front 
end. There the signal is detected by two orthogonal dipole antennas, preamplified and then sent to the backend, where further amplification is done and the spectra are formed. The reason the dipoles are orthogonal is to detect both polarizations of the radiation. The data are then collected and stored for future use. If both polarizations are used, they are typically stored separately. One typical form of data is spectral line data. Here, the power is measured across a range of frequencies, the bandwidth, and stored as measures of power versus frequency. The power levels detected in the raw spectral data can be from several sources, such as the sky and from the amplifiers. This typically produces a shape across the band that must be corrected for. See Figure C.1 for an example.

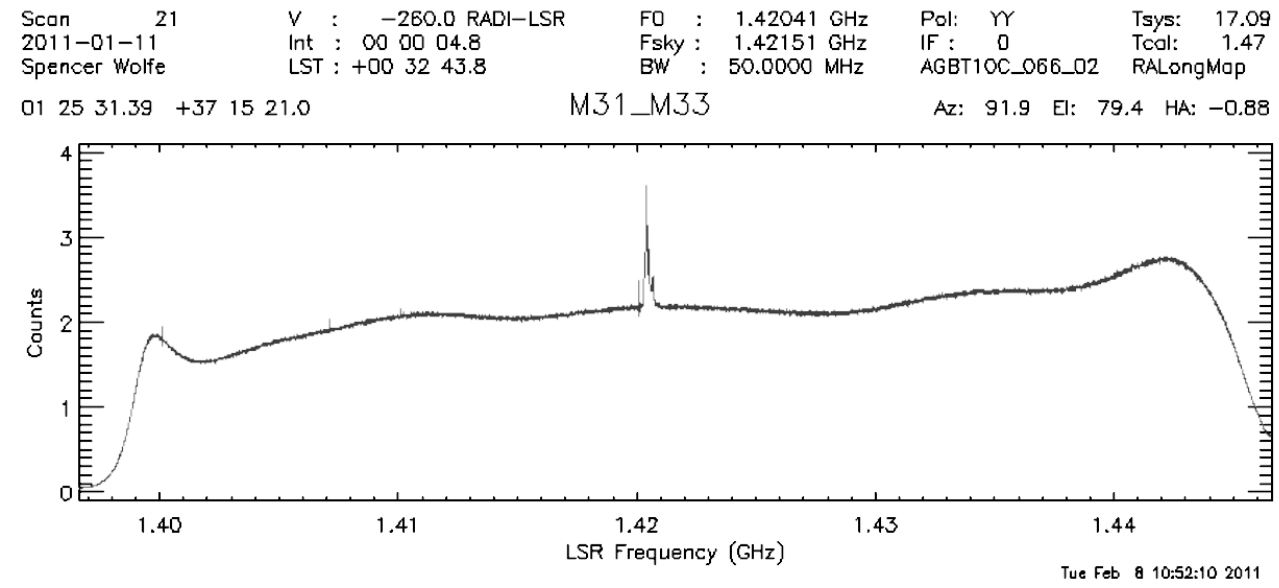

Figure C.1: A raw spectrum taken with the GBT. Note the broad shape seen across the spectral window. The spike in the middle at $1.42 \mathrm{GHz}$ is emission from our own Milky Way galaxy.

To subtract out the effects of the sky, electronics and so on, one generally takes two spectra. The first is a spectrum taken when the telescope is directly over the astronomical source of interest, or signal. The second is a reference spectrum which ideally contains all previous sources of input power except that of the astronomical source. There are two primary ways to do this. The first is to position switch, or take 
the reference spectrum at a different direction, but not far from the source. Thus when one subtracts the reference from the signal spectrum, all that should remain is the astronomical source. The second method is frequency switching, in which the reference spectrum is generated by shifting the central frequency of the spectral window by some amount. Frequency switching can be done quickly and is necessary when the source covers a large part of the sky, such as our own Galaxy, but because the shape of the band depends on frequency, the final spectra may still have residual structure. Position switching can produce less structure, but can take longer to do because the telescope must slew to a different position and then back again.

In either case, once the subtraction is done, the resulting spectra should be fairly flat with only the desired spectrum of the astronomical source remaining. See Figure C.2 for an example of position switching.

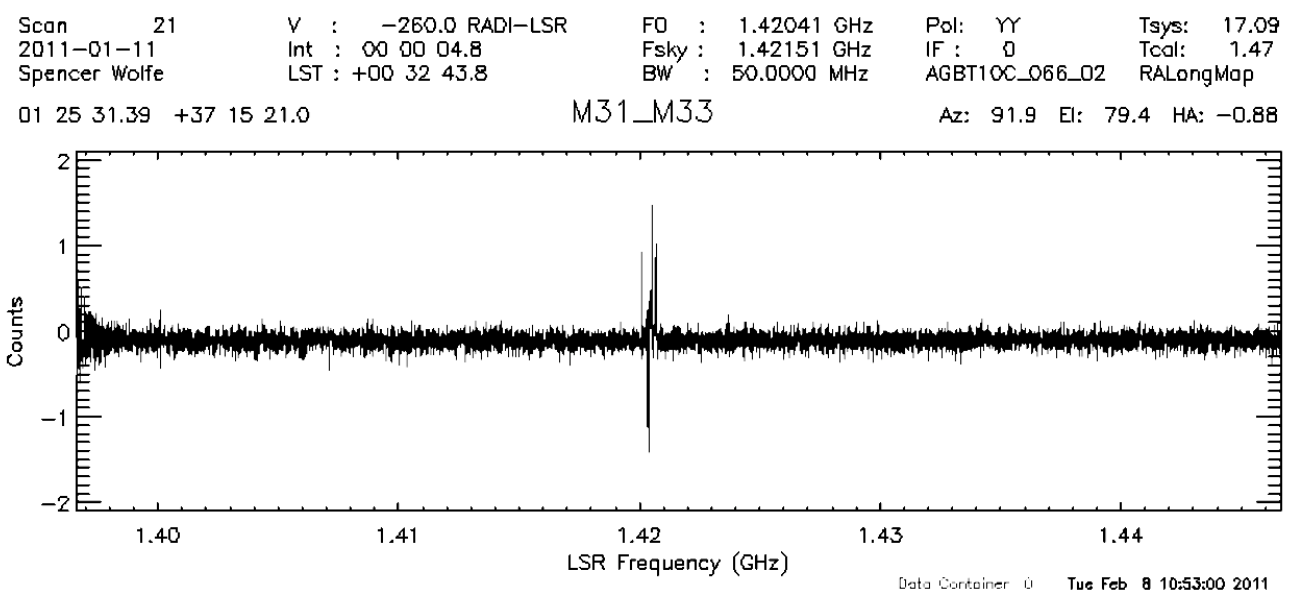

Figure C.2: A position switched spectrum. Notice how the spectral baseline is flat. The source of interest is too faint to see here. The residual features at $1.4 \mathrm{GHz}$ are incomplete subtractions of Milky Way emission since it is highly dependent upon position.

The spectra is still in terms of power, or counts, which should be converted to antenna temperature. Here the power $\mathrm{P}$ and temperature $\mathrm{T}$ are related by the 
gain $\mathrm{T}=\mathrm{GP}$. The temperature of the source then becomes the difference in power between the signal spectrum and reference spectrum

$$
\mathrm{T}_{\text {src }}=\mathrm{G}\left(\mathrm{P}_{\mathrm{sig}}-\mathrm{P}_{\mathrm{ref}}\right) .
$$

The gain is a difficult quantity to measure. The front end of the telescope has a noise diode that, when activated, injects power into the front end. The temperature of this, called $\mathrm{T}_{\text {cal }}$, is similarly the difference in power when the noise diode is on and off

$$
\mathrm{T}_{\text {cal }}=\mathrm{G}\left(\mathrm{P}_{\mathrm{CALon}}-\mathrm{P}_{\mathrm{CALoff}}\right)_{\text {ref }} .
$$

where the difference is taken at the reference position. Substituting Equation C.12 into C.13 gives

$$
\mathrm{T}_{\text {src }}=\mathrm{T}_{\text {cal }} \frac{\mathrm{P}_{\text {sig }}-\mathrm{P}_{\text {ref }}}{\left(\mathrm{P}_{\text {CALon }}-\mathrm{P}_{\text {CALoff }}\right)_{\text {ref }}} .
$$

To determine $\mathrm{T}_{\mathrm{cal}}$, one typically observes a calibration source of known flux density $\mathrm{S}_{\nu}$ taking both a signal and reference spectrum. For data specific to the Green Bank telescope (GBT) one uses the relation

$$
\mathrm{T}_{\text {cal }}=2.8 \eta_{\mathrm{a}} \mathrm{e}^{\frac{\tau}{\sin (\text { ell })}} \frac{\mathrm{P}_{\text {CALoff,sig }}-\mathrm{P}_{\text {CALoff,ref }}}{\mathrm{P}_{\text {CALon,ref }}-\mathrm{P}_{\text {CALoff,ref }}} .
$$

The factor of 2.8 comes from the GBT collecting area and Boltzmann's constant. el is the elevation, above the horizon, of the observation. $\tau$ is the atmospheric opacity at zenith $(\mathrm{el}=0)$ and is included to correct for attenuation of the signal as it passes 
through the atmosphere. Once $\mathrm{T}_{\text {cal }}$ is determined, it can then be applied to Equation C.14 to give the final spectrum in units of temperature versus frequency. 


\section{Bibliography}

Bajaja, E., Arnal, E. M., Larrarte, J. J., et al. 2005, A\&A, 440, 767

Barnes, D. G., Staveley-Smith, L., de Blok, W. J. G., et al. 2001, MNRAS, 322, 486

Beaton, R. L., Majewski, S. R., Guhathakurta, P., et al. 2007, ApJ, 658, L91

Bekki, K. 2008, MNRAS, 390, L24

Binney, J., \& Merrifield, M. 1998, Galactic Astronomy (Princeton University Press)

Bland-Hawthorn, J., Veilleux, S., Cecil, G. N., et al. 1998, MNRAS, 299, 611

Boothroyd, A. I., Blagrave, K., Lockman, F. J., et al. 2011, A\&A, 536, A81

Braun, R., \& Thilker, D. A. 2004, A\&A, 417, 421

Bregman, J. N. 1980, ApJ, 236, 577

Bregman, J. N. 2004, in Astrophysics and Space Science Library, Vol. 312, High Velocity Clouds, ed. H. van Woerden, B. P. Wakker, U. J. Schwarz, \& K. S. de Boer, 341

Brüns, C., \& Mebold, U. 2004, in Astrophysics and Space Science Library, Vol. 312, High Velocity Clouds, ed. H. van Woerden, B. P. Wakker, U. J. Schwarz, \& K. S. de Boer , 251

Brüns, C., Kerp, J., Staveley-Smith, L., et al. 2005, A\&A, 432, 45

Brunthaler, A., Reid, M. J., Falcke, H., Greenhill, L. J., \& Henkel, C. 2005, Science, 307,1440

Clausius, R. J. E. 1870, Philosophical Magazine, Series 4, 40, 122

Collins, J. A., Shull, J. M., \& Giroux, M. L. 2007, ApJ, 657, 271

Davé, R., Cen, R., Ostriker, J. P., et al. 2001, ApJ, 552, 473

de Heij, V., Braun, R., \& Burton, W. B. 2002, A\&A, 391, 159

Dekel, A., \& Birnboim, Y. 2006, MNRAS, 368, 2

Dove, J. B., \& Shull, J. M. 1994, ApJ, 423, 196

Draine, B. T. 2011, Physics of the Interstellar and Intergalactic Medium

Evans, N. W., \& Wilkinson, M. I. 2000, MNRAS, 316, 929

Ewen, H. I., \& Purcell, E. M. 1951, Nature, 168, 356

Fernández, X., Joung, M. R., \& Putman, M. E. 2012, ApJ, 749, 181 
Fixsen, D. J. 2009, ApJ, 707, 916

Ford, H. A., McClure-Griffiths, N. M., Lockman, F. J., et al. 2008, ApJ, 688, 290

Fukugita, M., \& Peebles, P. J. E. 2004, ApJ, 616, 643

Gentile, G., Famaey, B., Combes, F., et al. 2007, A\&A, 472, L25

Griffiths, D. 1999, Introduction to Electrodynamics, 3nd Edition (Upper Saddle River, NJ 07458: Pearson Education)

Grossi, M., Giovanardi, C., Corbelli, E., et al. 2008, A\&A, 487, 161

Hartmann, D., \& Burton, W. B. 1997, Atlas of Galactic Neutral Hydrogen (Cambridge University Press)

Hulsbosch, A. N. M., \& Wakker, B. P. 1988, A\&AS, 75, 191

Ibata, R., Martin, N. F., Irwin, M., et al. 2007, ApJ, 671, 1591

Kalberla, P. M. W., Burton, W. B., Hartmann, D., et al. 2005, A\&A, 440, 775

Kalberla, P. M. W., \& Dedes, L. 2008, A\&A, 487, 951

Kalberla, P. M. W., McClure-Griffiths, N. M., Pisano, D. J., et al. 2010, A\&A, 521, A17

Kereš, D., Katz, N., Fardal, M., Davé, R., \& Weinberg, D. H. 2009, MNRAS, 395, 160

Kereš, D., Katz, N., Weinberg, D. H., \& Davé, R. 2005, MNRAS, 363, 2

Leroy, A. K., Walter, F., Brinks, E., et al. 2008, AJ, 136, 2782

Lewis, G. F., Braun, R., McConnachie, A. W., et al. 2013, ApJ, 763, 4

Lockman, F. J. 2012, in American Astronomical Society Meeting Abstracts, Vol. 220, American Astronomical Society Meeting Abstracts 220, 433.10

Lockman, F. J., Benjamin, R. A., Heroux, A. J., \& Langston, G. I. 2008, ApJ, 679, L21

Lockman, F. J., Free, N. L., \& Shields, J. C. 2012, AJ, 144, 52

Lockman, F. J., Murphy, E. M., Petty-Powell, S., \& Urick, V. J. 2002, ApJS, 140, 331

Madau, P., Pozzetti, L., \& Dickinson, M. 1998, ApJ, 498, 106

Maller, A. H., \& Bullock, J. S. 2004, MNRAS, 355, 694

Maloney, P. 1993, ApJ, 414, 41 
Mangum, J. G., Emerson, D. T., \& Greisen, E. W. 2007, A\&A, 474, 679

McClure-Griffiths, N. M., Staveley-Smith, L., Lockman, F. J., et al. 2008, ApJ, 673, L143

McClure-Griffiths, N. M., Pisano, D. J., Calabretta, M. R., et al. 2009, ApJS, 181, 398

McConnachie, A. W., Irwin, M. J., Ibata, R. A., et al. 2009, Nature, 461, 66

McGaugh, S. S. 2012, AJ, 143, 40

McGaugh, S. S., Schombert, J. M., Bothun, G. D., \& de Blok, W. J. G. 2000, ApJ, 533, L99

McGaugh, S. S., \& Wolf, J. 2010, ApJ, 722, 248

Morras, R., Bajaja, E., Arnal, E. M., \& Pöppel, W. G. L. 2000, A\&AS, 142, 25

Moss, V. A., McClure-Griffiths, N. M., Murphy, T., et al. 2013, ApJS, 209, 12

Muller, C. A., Oort, J. H., \& Raimond, E. 1963, Academie des Sciences Paris Comptes Rendus, 257, 1661

Murphy, E. M., Lockman, F. J., \& Savage, B. D. 1995, ApJ, 447, 642

Nidever, D. L., Majewski, S. R., Butler Burton, W., \& Nigra, L. 2010, ApJ, 723, 1618

Oort, J. H. 1966, Bull. Astron. Inst. Netherlands., 18, 421

Peebles, P. J. E. 1995, ApJ, 449, 52

Popping, A., Davé, R., Braun, R., \& Oppenheimer, B. D. 2009, A\&A, 504, 15

Press, W. H., \& Schechter, P. 1974, ApJ, 187, 425

Prochaska, J. X., Herbert-Fort, S., \& Wolfe, A. M. 2005, ApJ, 635, 123

Putman, M. E., de Heij, V., Staveley-Smith, L., et al. 2002, AJ, 123, 873

Putman, M. E., Peek, J. E. G., Muratov, A., et al. 2009, ApJ, 703, 1486

Ribaudo, J., Lehner, N., Howk, J. C., et al. 2011, ApJ, 743, 207

Shankar, R. 1994, Principles of Quantum Mechanics, 2nd. Ed. (Springer)

Shapiro, P. R., \& Field, G. B. 1976, ApJ, 205, 762

Shull, J. M., Jones, J. R., Danforth, C. W., \& Collins, J. A. 2009, ApJ, 699, 754

Shull, J. M., Smith, B. D., \& Danforth, C. W. 2012, ApJ, 759, 23

Smith, G. P. 1963, Bull. Astron. Inst. Netherlands., 17, 203 
Sohn, S. T., Anderson, J., \& van der Marel, R. P. 2012, ApJ, 753, 7

Spitzer, L. 1978, Physical processes in the interstellar medium (New York WileyInterscience)

Spitzer, Jr., L. 1956, ApJ, 124, 20

Stanimirovic, S., Altschuler, D., Goldsmith, P., \& Salter, C., eds. 2002, Astronomical Society of the Pacific Conference Series, Vol. 278, Single-Dish Radio Astronomy: Techniques and Applications

Stanimirović, S., Hoffman, S., Heiles, C., et al. 2008, ApJ, 680, 276

Stocke, J. T., Keeney, B. A., \& Danforth, C. W. 2010, PASA, 27, 256

Thilker, D. A., Braun, R., Walterbos, R. A. M., et al. 2004, ApJ, 601, L39

Tollerud, E. J., Beaton, R. L., Geha, M. C., et al. 2012, ApJ, 752, 45

Tully, R. B., \& Fisher, J. R. 1977, A\&A, 54, 661

van der Marel, R. P., Besla, G., Cox, T. J., Sohn, S. T., \& Anderson, J. 2012, ApJ, 753,9

van Woerden, H., Rougoor, G. W., \& Oort, J. H. 1957, Academie des Sciences Paris Comptes Rendus, 244, 1691

van Woerden, H., Wakker, B. P., Schwarz, U. J., \& de Boer, K. S., eds. 2004, Astrophysics and Space Science Library, Vol. 312, High Velocity Clouds

Venzmer, M. S., Kerp, J., \& Kalberla, P. M. W. 2012, A\&A, 547, A12

Voskes, T., \& Burton, W. B. 1999, in Astronomical Society of the Pacific Conference Series, Vol. 168, New Perspectives on the Interstellar Medium, ed. A. R. Taylor, T. L. Landecker, \& G. Joncas, 375

Wakker, B. P. 1990, PhD thesis, Univ. Groningen

—. 2001, ApJS, 136, 463

Wakker, B. P. 2004, in Astrophysics and Space Science Library, Vol. 312, High Velocity Clouds, ed. H. van Woerden, B. P. Wakker, U. J. Schwarz, \& K. S. de Boer, 25

Wakker, B. P., \& van Woerden, H. 1991, A\&A, 250, 509

Wakker, B. P., Howk, J. C., Savage, B. D., et al. 1999, Nature, 402, 388

Wannier, P., Wrixon, G. T., \& Wilson, R. W. 1972, A\&A, 18, 224

Westmeier, T. 2007, PhD thesis, Rheinische Friedrich-Wilhelms-Universität Bonn 
Westmeier, T., Brüns, C., \& Kerp, J. 2005, A\&A, 432, 937

—. 2008, MNRAS, 390, 1691

White, S. D. M., \& Frenk, C. S. 1991, ApJ, 379, 52

Wilson, T. L., Rohlfs, K., \& Hüttemeister, S. 2009, Tools of Radio Astronomy (Springer-Verlag)

Witt, C. M., Wakker, B., Engel, T. D., et al. 2011, in Bulletin of the American Astronomical Society, Vol. 43, American Astronomical Society Meeting Abstracts \#217, 251.30

Wolfe, S. A., Pisano, D. J., Lockman, F. J., McGaugh, S. S., \& Shaya, E. J. 2013, Nature, 497, 224

Wolfire, M. G., Hollenbach, D., McKee, C. F., Tielens, A. G. G. M., \& Bakes, E. L. O. 1995, ApJ, 443, 152 